\title{
Nearfield Summary and Statistical Analysis of the Second AIAA Sonic Boom Prediction Workshop
}

\author{
Michael A. Park, ${ }^{*}$ \\ NASA Langley Research Center, Hampton, VA 23681
}

\author{
Marian Nemec ${ }^{\dagger}$ \\ NASA Ames Research Center, Moffett Field, CA 94035
}

\begin{abstract}
A summary is provided for the Second AIAA Sonic Boom Workshop held 8-9 January 2017 in conjunction with AIAA SciTech 2017. The workshop used three required models of increasing complexity: an axisymmetric body, a wing body, and a complete configuration with flow-through nacelle. An optional complete configuration with propulsion boundary conditions is also provided. These models are designed with similar nearfield signatures to isolate geometry and shock/expansion interaction effects. Eleven international participant groups submitted nearfield signatures with forces, pitching moment, and iterative convergence norms. Statistics and grid convergence of these nearfield signatures are presented. These submissions are propagated to the ground, and noise levels are computed. This allows the grid convergence and the statistical distribution of a noise level to be computed. While progress is documented since the first workshop, improvement to the analysis methods for a possible subsequent workshop are provided. The complete configuration with flow-through nacelle showed the most dramatic improvement between the two workshops. The current workshop cases are more relevant to vehicles with lower loudness and have the potential for lower annoyance than the first workshop cases. The models for this workshop with quieter ground noise levels than the first workshop exposed weaknesses in analysis, particularly in convective discretization.
\end{abstract}

\section{Introduction}

The Second American Institute of Aeronautics and Astronautics (AIAA) Sonic Boom Prediction Workshop (SBPW2) was held on January 7-8, 2019 at Grapevine, Texas. This workshop was a sequel to the First AIAA Sonic Boom Prediction Workshop (SBPW1), which was documented by Park and Morgenstern [1]. The first day of SBPW2 focused on analysis of the nearfield pressure field and is covered here. The second day focused on propagation as detailed by Rallabhandi and Loubeau [2]. There were approximately 50 attendees. The objective of the first day of this workshop was to assess the state of the art for predicting nearfield pressure signatures needed for accurate and reliable sonic boom prediction. Nearfield pressure signatures, extracted from Computational Fluid Dynamics (CFD) solutions, were gathered from the international participants. The participants were required to use a series of uniformly-refined, workshop-provided grids. Participants also created grids with each participant's best practices for computing nearfield pressure signataures on the provided geometries.

Statistical analysis of the SBPW2 submissions is facilitated by reducing the submitted nearfield signatures to a set of scalars (loudness levels) for $\mathrm{N}$-version testing [3]. The statistical products and grid convergence studies continue the process of quantifying the uncertainty of nearfield CFD employed by the international participants. SBPW2 benefits from the lessons learned at the 2008 NASA Sonic Boom Prediction Workshop [4] and SBPW1 [1,5]. Experience from AIAA Drag [6,7], High Lift [8], and Shock Boundary Layer Interaction [9] Prediction Workshops also contributed to the success of the current workshop.

A website (https: / / lbpw. larc. nasa.gov) and a companion FTP server were used to organize the workshop and disseminate results. This includes all the participant presentations, computational grids, and submitted results to encourage continued research. Workshop participants also published details of their analysis in conference papers organized into an invited special session at the AIAA AVIAITON 2017 conference with this summary [2, 10-12].

The data and analysis methods of this summary are intended to contribute toward the discussion of replacing the prohibition of overland supersonic flight [13] with a certification standard. The existing ban on overland flight increases the cost and block time [14] of supersonic fights between city pairs and is an important driver of the economic viability of a supersonic transport $[15,16]$.

\section{Evaluation Methods}

SBPW2 focuses on nearfield CFD, but predicting the acoustic signature on the ground and how it is perceived by humans is the goal of the sonic boom community. The SBPW2 participants submitted required nearfield pressure sig-

\footnotetext{
*Research Scientist, Computational AeroSciences Branch, Mike.Park@NASA.gov, AIAA Senior Member.

${ }^{\dagger}$ Aerospace Engineer, Computational Aerosciences Branch, Marian.Nemec@NASA.gov, AIAA Senior Member.
} 
natures. While these signatures could be compared directly to each other, Reducing the properties of these signatures to scalars enables grid-refinement studies and the application of statistical methods. The submitted nearfield signatures are propagated to the ground (Fig. 1), and noise levels are computed to reduce these signatures to relevant scalars. The

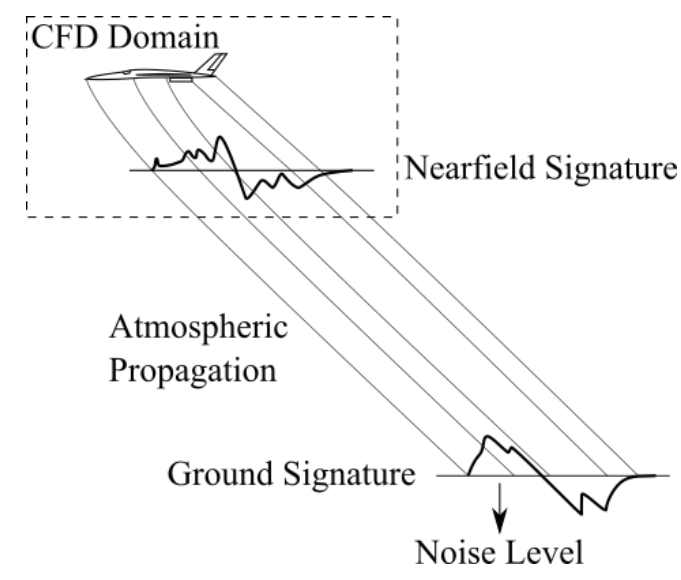

Figure 1. Sonic boom prediction process.

evaluation methods are detailed in Park and Morgenstern [1,5]. This data reduction was performed by the authors, not by the participants, in an attempt to apply these methods as uniformly as possible to the submissions. Participants optionally provided ground signatures and loudness levels with independent methods. These optional submissions are available to verify the uniformly applied process.

\section{A. Signal Ensemble Mean and Standard Deviation}

The participants used a variety of methods to compute the submitted signatures. These submissions form an ensemble. Examining the pointwise mean and standard deviation of these signatures highlights the portions of the signature with a large variation and portions with a small variation. A similar technique is used in wind tunnel testing, where signatures measured at different locations in a wind tunnel test section are spatially averaged to reduce the impact of disturbances in the tunnel $[17,18]$.

The mean and standard deviation are computed by interpolating the finest-grid submission from each case to a common set of points with $1 \mathrm{~cm}$ resolution or 3292 points per body length. This variation among the ensemble of measurements is expressed as a standard deviation at each interpolated point. The interpolated mean is shown as a line, and the standard deviation is shown as an error bar with a height of one standard deviation.

\section{B. Grid Convergence}

Most participants submitted three or more nearfield signatures obtained from their grid refinement studies. To provide an assessment of the signature's grid convergence, a procedure based on Richardson extrapolation is used to obtain a pointwise discretization error estimate. The goal is to check if the sequence of signatures submitted by each participant is convergent and to identify regions of the signature with the greatest sensitivity to grid refinement.

The error-estimation procedure is described in detail in Anderson et al. [12]. Given signatures from a coarsemedium-fine grid sequence, the procedure computes the L1-norms of the differences between the coarse-medium and medium-fine signature pairs. Linear interpolation is used to sample the data from the higher-resolution solutions to the lower-resolution point set. An observed order of accuracy [19] is calculated from the two L1-norms and the grid refinement ratios inferred from the total number of control volumes in each grid. The observed order of accuracy is assumed to be constant over the signature and is applied pointwise to obtain a local discretization error estimate.

Note that this error estimate does not reliably predict the error bounds on the extrema of the signature and is sensitive to phase shifts. Nevertheless, the error estimate provides a reliable qualitative assessment of the behavior of the signature due to grid refinement, and efficiently identifies grid sequences and solvers with poor spatial convergence characteristics. This asymptotic convergence analysis was performed only on cases with signatures on three or more grids. 


\section{Noise Levels}

Sonic boom loudness and annoyance are an inherently subjective experience. Many noise descriptors have been evaluated as loudness predictors of sonic booms in human experiments, see Leatherwood et al. [20]. The Mark VII perceived level (PL) of Stevens [21] is used to compare submissions because it is correlated with loudness and annoyance in multiple experiments [20,22]. The method of Shepherd and Sullivan [23] is used to calculate PL from ground signatures.

\section{Propagation}

The sBOOM code of Rallabhandi [24] solves an augmented Burgers' equation to propagate nearfield signatures to the ground. This is the atmospheric propagation step in Fig. 1. Rallabhandi [25] provides additional details of sBOOM including an adjoint formulation [26]. Version 2.4.0 of sBOOM is used in this summary.

Like the results of any finite-difference method [27], the ground signatures predicted by sBOOM have a sensitivity to the number of points used to discretize the signature [24]. For example, to verify the consistency of the sBOOM finite difference method, a series of uniformly-refined grids are used in Park and Morgenstern [5]. This reference also examines frequency spectra showing that SBOOM grid refinement has the greatest impact on the higher frequencies of the ground signature.

These full-scale vehicles are assumed to be at $15,760 \mathrm{~m}$ altitude in a no-wind US Standard Atmosphere [28] and the atmospheric humidity model from ANSI S1.26, Annex C [29]. The specified Mach number is 1.6 for all cases. The grids are truncated before the aft pressure signature returns to freestream to reduce computational cost. Participants provided extracted pressure between different forward and aft locations. The influence of these submission differences is reduced by windowing the signatures. Before propagation, the initial and final delta pressures are linearly ramped to zero, windowing the submission to focus the statistics on the model. The fore and aft signature ramping is applied uniformly based on the freestream Mach cone extending from the nose of each model. The forward portion of signature is ramped from zero to the submitted pressure between -0.2 and -0.1 body lengths ahead of the freestream Mach cone. The aft portion of signature is ramped from the submitted pressure to zero between 1.5 and 1.8 body lengths behind of the freestream Mach cone. The windowed nearfield signature is padded with zeros to have a full extent of -0.5 to 2.5 body lengths from the freestream Mach cone. The sBOOM discretization uses 36,000 points to propagate the signature, which is 12,000 points per body length or 365 points per meter in the nearfield. This spatial resolution near the aircraft results in $172 \mathrm{kHz}$ temporal resolution at the ground. This temporal resolution is a slightly higher than SBPW1, which used 90, 100, and $161 \mathrm{kHz}[1,5]$.

Acoustic disturbance sources distributed in the spanwise direction reach a nearfield extraction location after traveling a longer distance than the centerline disturbances. This extra distance results in wave superposition at a more aft location that alters the signature shape. This signature change with extraction distance is known as nearfield interference. When signatures are extracted sufficiently far from the model, a farfield propagation technique (sBOOM) is usable without farfield correction [30] because the acoustic sources appear to come from a single line. This workshop extracts nearfield signatures at different distances to investigate the impact of nearfield interference.

\section{E. Statistical Approach and Scatter Limits}

The intention of this applied statistical approach is to measure the repeatability of an experiment that is run multiple times, where a workshop submission is treated as an experiment. In this context, no submission is treated as the "correct" prediction because all submissions contain known and unknown sources of uncertainty. The approach is based on the principles and methods of the AIAA Drag Prediction Workshop (DPW) statistical summary [3] and uses the descriptive statistics approach of Derlaga and Morrison [31]. Box and whisker plots [32] are combined with violin plots [33] to represent an estimate of the sample probability distribution. The violin plots can indicate details of the probability distribution such as bimodality or skew. The upper and lower box plot extents denote the interquartile range (IQR), which contains half of the samples. The median is the line interior to the box, and the diamond is the sample mean. The dashed lines are the whiskers that extend 1.5 times the height of the IQR or the last sample, whichever is closest to the median. Samples of a normal distribution would have a 95\% likelihood of being within the whisker plots, which is approximately two standard deviations from the mean. Samples that are outside of the whiskers are plotted as filled circles.

\section{Participants}

The groups contributing to the workshop are listed in Table 1. The group letter identifier is included in the submission identifier throughout this summary. Some groups contain a single contributor, but most contain two or more collab- 
orators. Government agencies, industry, and academia are represented. Each group submitted results and prepared a presentation for the workshop. All group presentations and data submissions are available on the workshop website lbpw.larc.nasa.gov along with the workshop introduction, overview, and summary presentations. Some groups published details of their analysis in conference papers, which are identified in the methods section.

Table 1. Participant groups.

\begin{tabular}{rll} 
Group & Organizations & Contributors \\
\hline A & DLR & J. Kirz and R. Rudnik \\
B & NW Polytechnical University & G. Wand, B. Ma, and Z. Wang \\
C & NASA & M. Park \\
D & ASIRI, JAXA, and U. Tokyo & H. Ishikawa, Y. Makino, A. Ueno, and Y. Kasuga \\
E & NASA & J. Housman, J. Jensen, M. Denison, and C. Kiris \\
F & STC and NASA & G. Anderson and M. Aftosmis \\
G & Gulfstream & D. Howe, A. Clemens, and M. Wintzer \\
H & Lockheed Martin & F. Marconi and J. Morgenstern \\
I & NASA, AS\&M, and U. Vermont & M. Carter, A. Elmiligui, S. Cliff, S. Nayani, and J. Pearl \\
J & Boeing & T. Magee and D. Lazzara \\
K & INRIA & A. Loseille, L. Frazza, and F. Alauzet \\
\hline
\end{tabular}

Participant A used the DLR-TAU code [34]. DLR-TAU has also been applied to the SBPW1 cases [35]. Details of the DLR-TAU application to SBPW2 are available in [10]. Two reconstruction limiters were used: DLR-TAU-V used the Venkatakrishnan limiter [36] and DLR-TAU-BJ used the Barth-Jespersen limiter [37]. Participant B used HUNS3D solver, which has been applied to the SBPW1 cases [38]. Participants C, G, and J used the FUN3D [39] solver. Four convection schemes were used: FUN3D used unlimited reconstruction with the Roe flux [40], FUN3D-VA used a van Albada [41] with the Roe flux, FUN3D-VL used a van Leer limiter [42] with the Roe flux, FUN3D-VL-HLLC used a van Leer limiter HLLC flux [43].

Participant D used the JTAS [44] flow solver with Venkatakrishnan limiter and HLLEW (Harten-Lax-van LeerEinfeldt-Wada) flux. Participant E used LAVA [45] on unstructured and structured-overset grid systems. LAVA used a second-order modified Roe scheme on unstructured grids and a sixth-order Hybrid Weighted Compact Nonlinear Scheme (HWCNS) with second-order viscous fluxes on overset grid systems. LAVA was also used in SBPW1 [46]. Participants F and J used Cart3D [47], which was applied to SBPW1 [47] and includes an output-based grid adaptation scheme. Further details are available for the Participant F submission to SBPW2 [12]. Participant G used CartOVER [48], which combines Cart3D near the body with an OVERFLOW collar grid.

Participant H used CFD++ version 12.1.1 [49]. Participant I used USM3D with the minmod limiter [50]. Details of the USM3D application to SBPW2 are available with additional cases not submitted to the workshop [11]. Participant $\mathrm{K}$ used the Wolf [51] solver and applied adaptive methods to the SBPW2 cases [52]. Wolf was also used in SBPW1 [53]. Wolf-V6 uses a sixth-order numerical dissipation scheme [54].

Two turbulence models are used in the Reynolds-averaged Navier-Stokes (RANS) simulations. The SpalartAllmaras (RANS-SA) [55,56] turbulence model is used by most participants and the Realizable $k-\epsilon$ (RANS-RKE) [57] is used by participant $\mathrm{H}$. 


\section{Models}

The models for SBPW2 were chosen to provide a range of geometric complexities, see Fig. 2. Three cases were required: Axisymmetric Equivalent Area (AXIE), JAXA Wing Body (JWB), NASA Concept 25D with Flow-through nacelle $(\mathrm{C} 25 \mathrm{~F})$. One optional case was the NASA Concept 25D with Powered nacelle (C25P). They are also chosen to produce similar signatures at three body lengths below the centerline. This allows an evaluation of a variable pressure field and geometric complexity on a set of models with similar nearfield signatures.

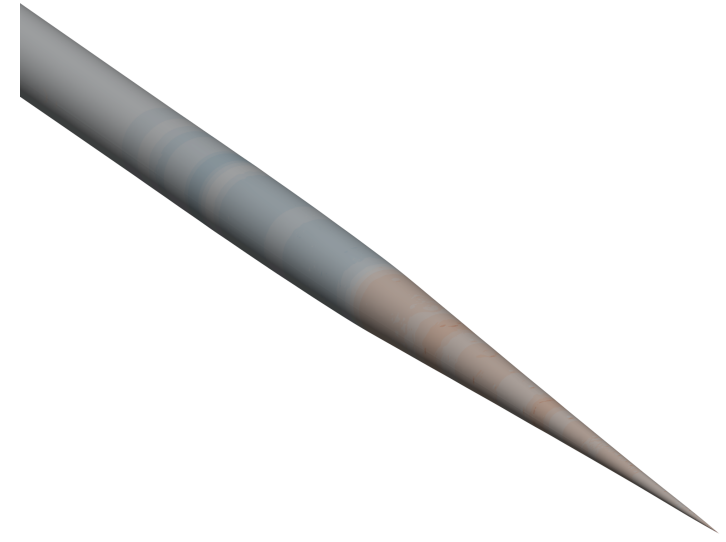

a) AXIE.

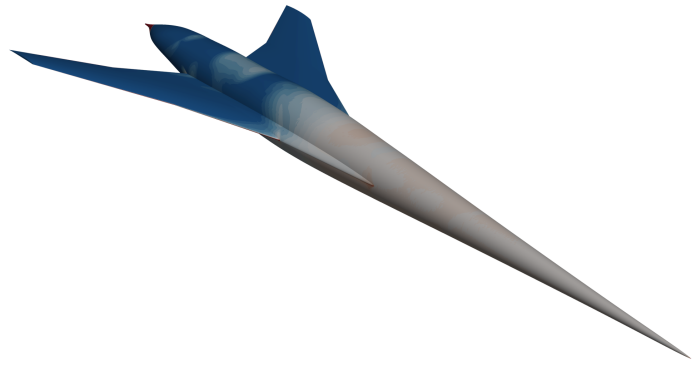

b) JWB.

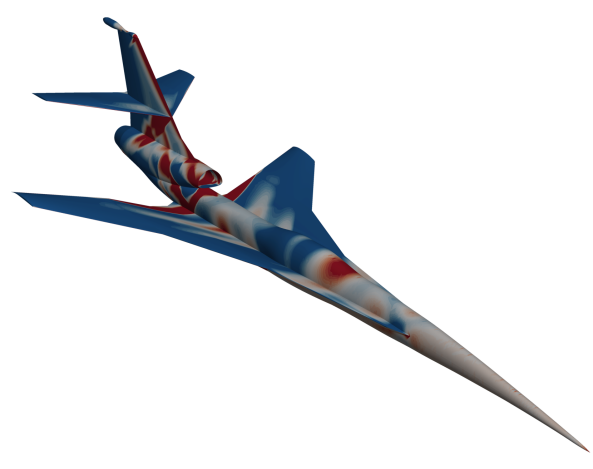

c) $\mathrm{C} 25 \mathrm{~F}$.

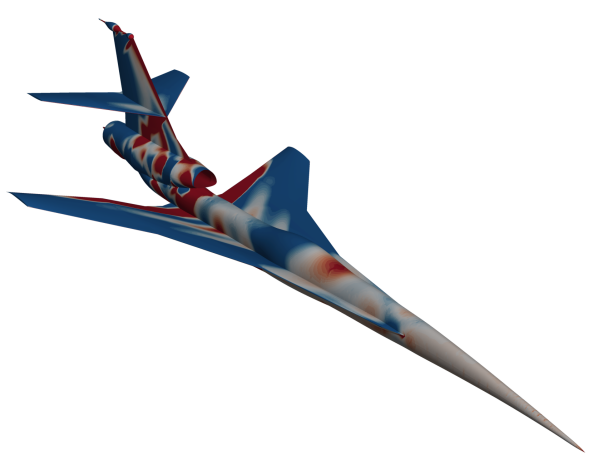

d) C25P.

Figure 2. Model geometry colored with pressure difference, shown in the same scale and perspective.

The signatures at three body lengths are shown as the mean of fine grid participant signatures in Fig. 3. The $x$ axis variable TAU is the distance from the freestream Mach cone emanating from the nose. The distance TAU (also labeled $\tau$ ) is normalized by the reference body length. The $y$-axis is the pressure difference from freestream divided by freestream pressure. This pressure difference is scaled by the square root of the radius in reference body lengths to form Delta Pressure Square Root (DPSR, also labeled $\Delta p / p_{\infty} \sqrt{R / L}$ ). The variable $\mathrm{N}$ in the legend is the number of submissions that contribute to the mean. These four models produce the intended similarity in the nearfield pressure signature magnitude at three body lengths on the centerline and contain similar frequency content.

\section{A. C25F and C25P}

The $\mathrm{C} 25 \mathrm{~F}$ is a notional configuration created to represent a sonic boom demonstrator class vehicle. This case is the required complex configuration. This full configuration model includes wing, body, tail, nacelle, and flow-through engine path. It was designed primarily for a low ground loudness level near the centerline of the flightpath [58], with some reduction in the noise level over the entire boom carpet [59]. The Euler method Cart3D was used to design the configuration. The geometry is provided in the correct incidence for $0^{\circ}$ angle of attack. The freestream Mach number 


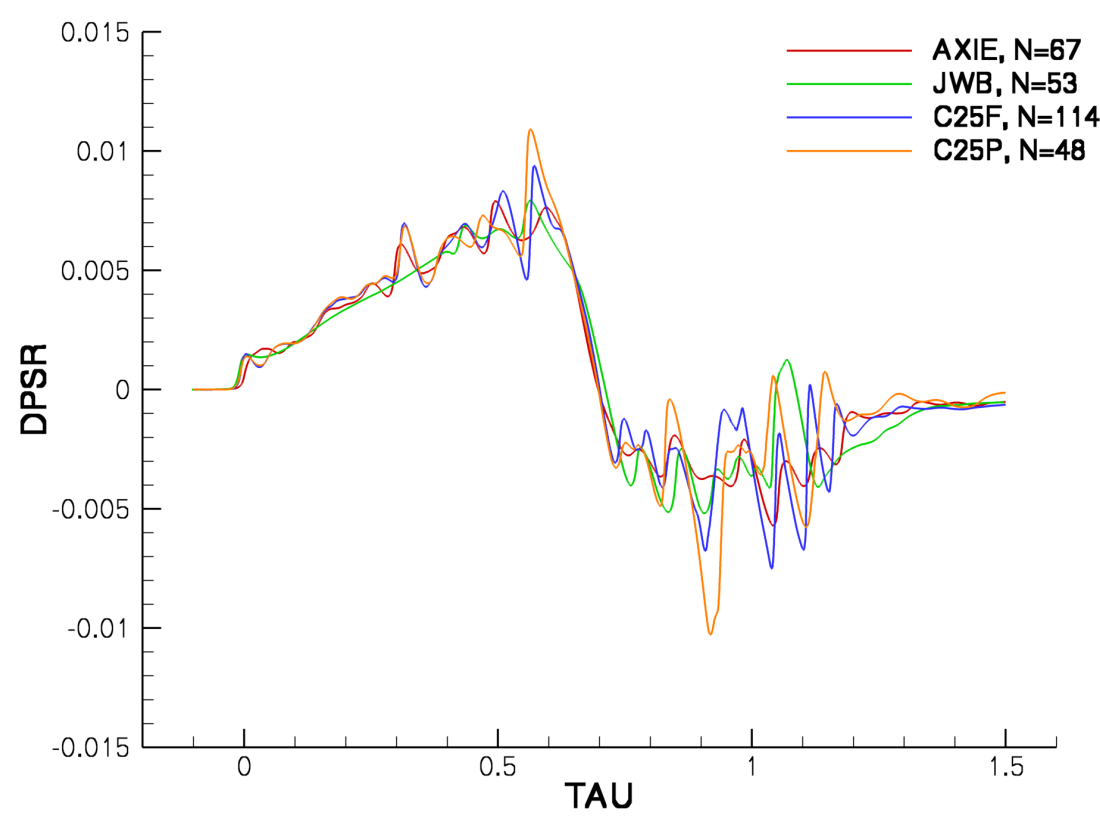

Figure 3. Mean nearfield signatures at three body length for all submissions.

is 1.6 at an altitude of $15,760 \mathrm{~m}$. The workshop committee requested turbulent calculations at a unit Reynolds number of 5.70 million per meter for viscous calculations.

The Euler solution on the $\mathrm{C} 25 \mathrm{~F}$ symmetry plane is shown in Fig. 4. There are complex shock and expansion interactions present. Derlaga, Park, and Rallabhandi [60] provide details on how the inlet shock is reflected from the upper wing surface and lower horizontal tail surface to impact the nearfield and propagated ground signature.

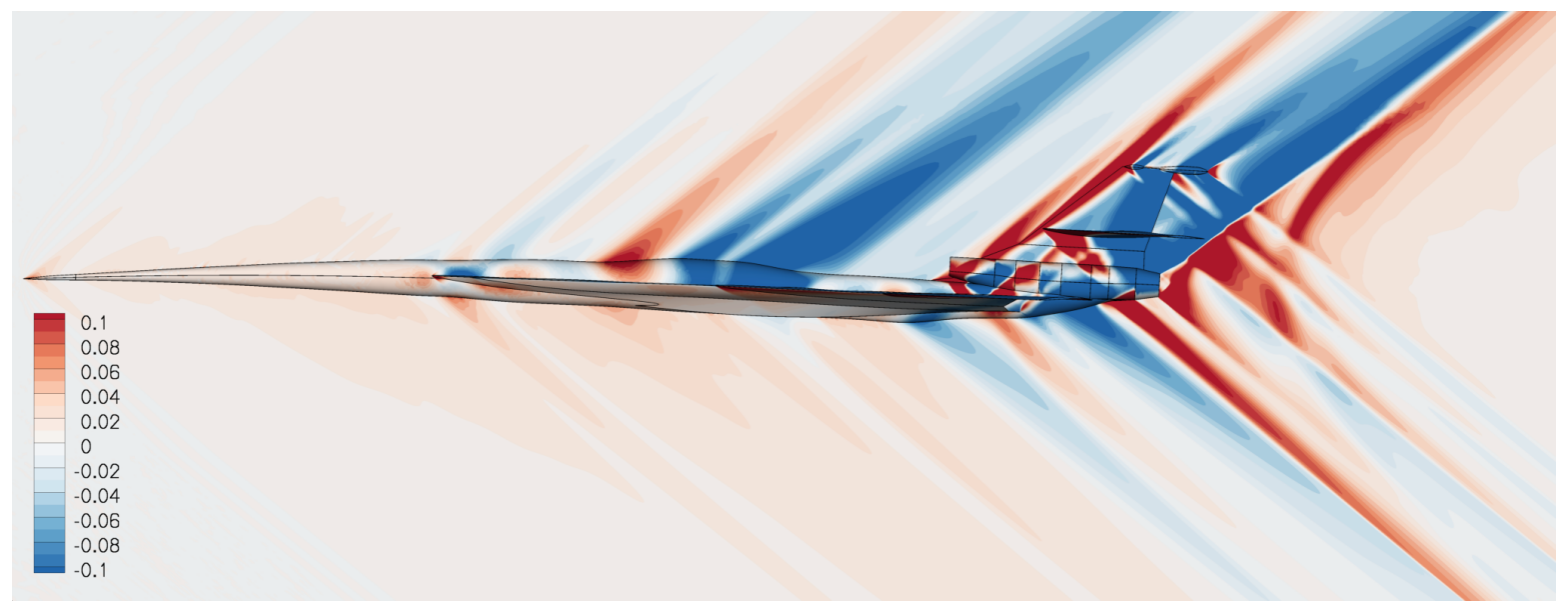

Figure 4. C25F symmetry plane pressure difference normalized with freestream pressure.

The $\mathrm{C} 25 \mathrm{P}$ is a notional configuration created to represent a sonic boom demonstrator class vehicle. This case is the optional complex configuration with propulsion boundary conditions. This full configuration model includes wing, body, tail, nacelle, and engine interface surfaces. It is based on the $\mathrm{C} 25 \mathrm{~F}$ and redesigned to lower ground loudness level after activating power boundary conditions [58]. The Euler method Cart3D was used to design the configuration. The geometry is provided in the correct incidence for $0^{\circ}$ angle of attack. The freestream Mach number is 1.6 at an altitude of $15,760 \mathrm{~m}$. The workshop committee requested turbulent calculations at a unit Reynolds number of 5.70 million per meter for the viscous calculations. The engine fan face static pressure ratio to freestream static pressure 
is 3.2606. The engine plenum total pressure ratio to freestream static pressure is 14.540 . The engine plenum total temperature ratio to freestream static temperature is 7.8722 .

The complexity of the nearfield pressure field of the $\mathrm{C} 25 \mathrm{P}$ is shown by the Euler symmetry plane solution in Fig. 5. The oblique inlet shock is stronger than the C25F, with an offset normal shock. Slight geometry changes are introduced to reduce ground loudness while accommodating the powered boundary conditions. The geometry changes for the $\mathrm{C} 25 \mathrm{P}$ increase the high frequency content of the shock and expansion interaction. The influence of the plume can bee seen in the shocks and expansion propagating downward from the tail features.

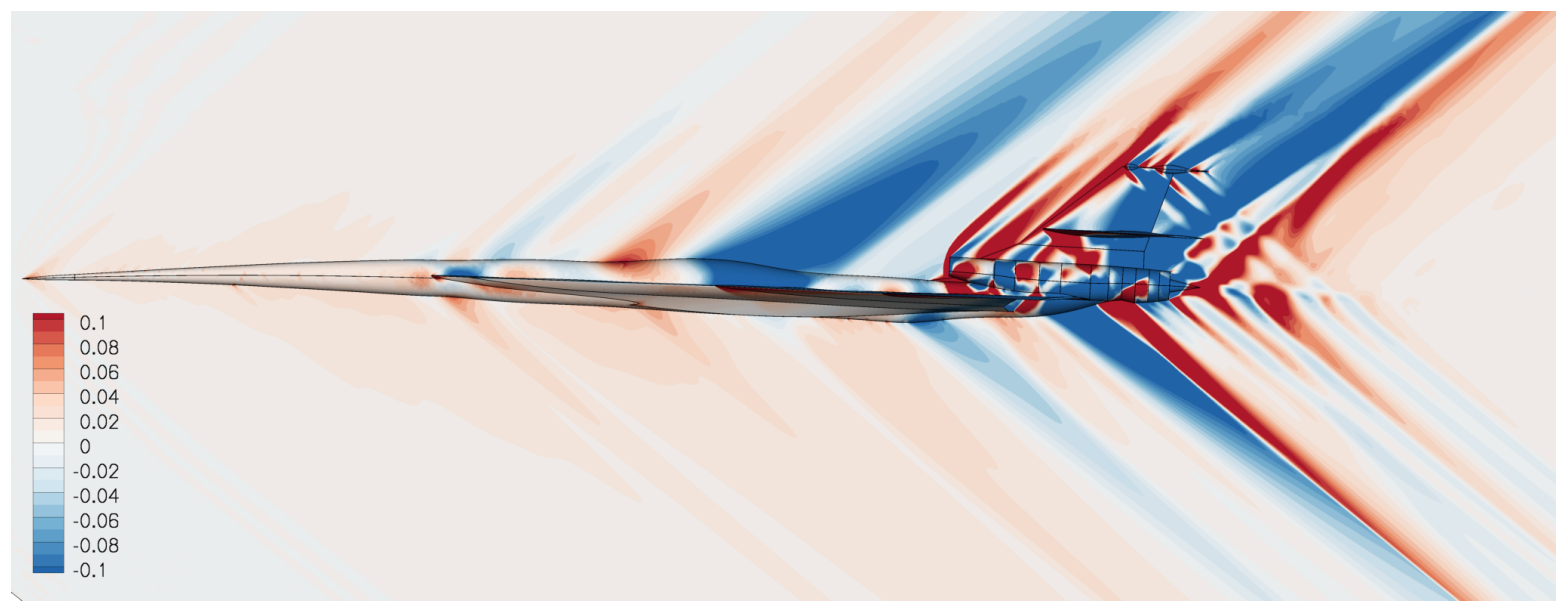

Figure 5. C25P symmetry plane pressure difference.

The geometry for C25F and C25P was created by an OpenCSM [61] script that translated the Jaguar [62] description used to develop the model. The OpenCSM model is not a perfect recreation of the Jaguar model. The OpenCSM nacelle outer mold line is linearly interpolated between fuselage stations where the Jaguar model is splined. There are other smaller known differences that result in different loudness levels in these workshop configurations than AIAA papers describing the design. The translation was performed to create STEP geometry, which is a common input for a number of the participant methods. The provided geometry is oriented to include the design angle of attack of $3.375^{\circ}$,

The body length is $32.92 \mathrm{~m}$ for both the $\mathrm{C} 25 \mathrm{~F}$ and $\mathrm{C} 25 \mathrm{P}$. Nearfield pressure was requested at one, three, and five body lengths below the model at off-track angles of $0^{\circ}, 10^{\circ}, 20^{\circ}, 30^{\circ}, 40^{\circ}$, and $50^{\circ}$.

\section{B. JWB}

The JWB design process is described by Ueno, Kanamori, and Makino [63]. The JWB is a wing body configuration designed to the same equivalent area target as the NASA Concept 25D [64]. The first phase of the design used Euler calculations and shape optimization to match the equivalent area target. The second phase used reversed equivalent area based optimization with Euler and multipole analysis.

The nearfield pressure field of the JWB is shown as the Euler symmetry plane solution in Figure 6. This configuration has a strong expansion surrounding the lower fuselage closeout that is intended to interfere with the shock wave at the rear fuselage [63]. The details of this strong expansion and its interaction with the rear fuselage shock will be shown to be significantly different for RANS simulations.

The body and wing geometries were exported from CATIA as IGES surfaces. These surfaces were combined into a solid model in NX, rotated to include the design angle of attack of $2.3067^{\circ}$, and exported as STEP files. Optional turbulent calculations were requested at a flight unit Reynolds number of 5.70 million per meter for the viscous calculations. A wind tunnel test of the model is also planned at a scaled unit Reynolds number of 132,000 per meter.

The original problem description specified a body length of $38.7 \mathrm{~m}$ and extraction locations of 0.85 an 2.55 body lengths at off-track angles of $0^{\circ}, 10^{\circ}, 20^{\circ}, 30^{\circ}, 40^{\circ}$, and $50^{\circ}$. For the workshop and this summary, a $32.92 \mathrm{~m}$ body length and extraction location of one and three body lengths that is common with the other models is used. Pressure signatures were not requested at five body lengths due to the extent of the committee-provided grids as described in section V. The use of a common description of body lengths and extraction locations for all models simplified the analysis of submissions and plotting. 


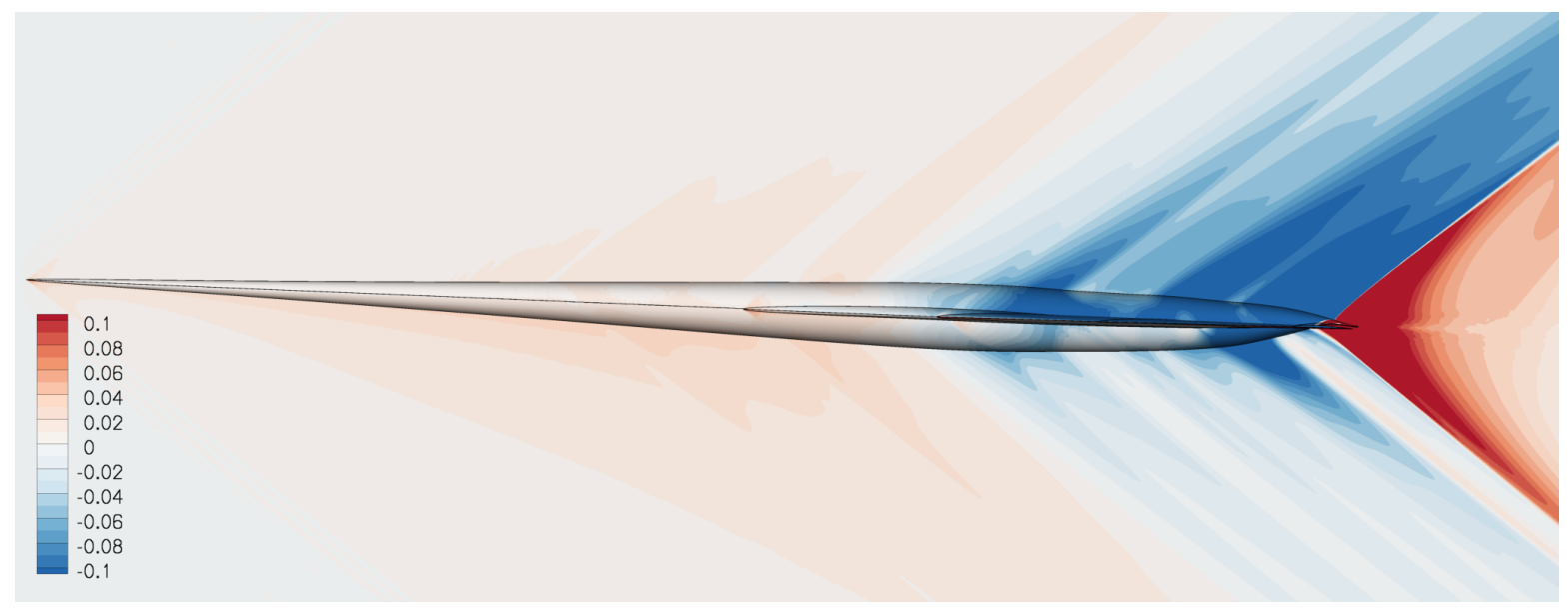

Figure 6. JWB symmetry plane pressure difference.

\section{AXIE}

The AXIE geometry is created by an inverse design process described by Anderson [65]. It is intended to have the same pressure signature as $\mathrm{C} 25 \mathrm{~F}$ at three body lengths on the centerline. The geometry is designed in Jaguar [62]. The Jaguar geometry is interrogated to compute the radius as a function of distance from the nose. This radius function was truncated at 1.85 reference body lengths and modified to add a length of 0.93 body lengths of constant radius. The model closure is a cosine function over the last 0.93 body lengths. The total length of the geometry is 3.70 body lengths. The modified radius function was processed by an OpenCSM script to create a solid model exported as STEP. The model has a reference length of $32.92 \mathrm{~m}$. The participants provided submissions at one, three, and five body lengths below the centerline of the model.

The nearfield pressure field of the AXIE is shown as the Euler symmetry plane solution in Figure 7. The geometry makes very weak pressure perturbations on this color scale, which is the same range as the other three configurations. This case allows for a focus on signature propagation in the nearfield without the complexity of the winged configurations.

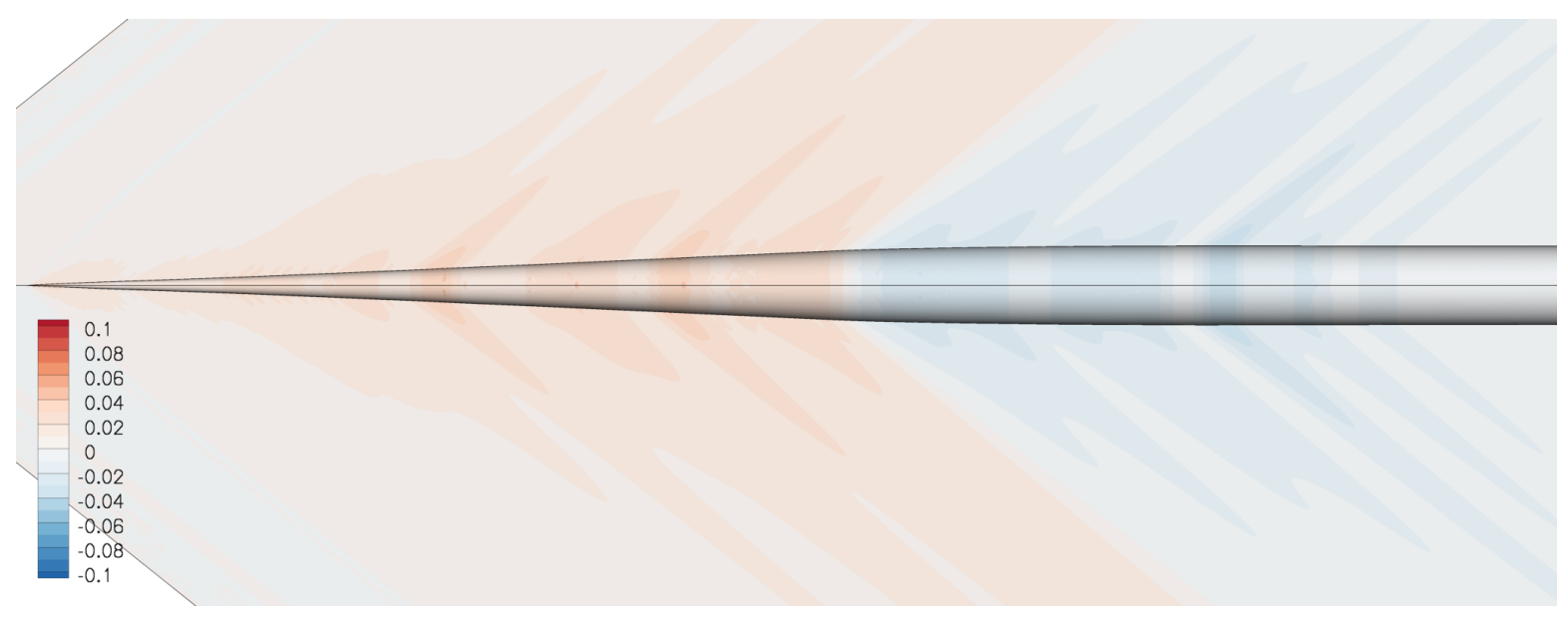

Figure 7. AXIE symmetry plane pressure difference. 


\section{Required Grids}

The provided unstructured mixed-element and tetrahedral-only grids for the AXIE, C25F, and C25D were created with the method of Park et al. [66]. The JWB grids used the HeldenMesh tool. A series of uniformly-refined grids were provided to quantify the spatial convergence of the nearfield CFD schemes. The wide range of grid resolutions includes grids that were practical for both node-centered and cell-centered schemes. Care was taken to ensure that the relative grid spacing distribution remained as similar as possible for the unstructured family of grids. The number of nodes and elements approximately doubled, and a characteristic grid spacing decreased by the cube root of one half between successive grids in the series [66]. Baseline grid spacing recommendations are provided for the $\mathrm{C} 25 \mathrm{~F}$ and C25P in Appendix A: C25D and C25F Grid Generation Guidelines.

Participants also provided grids, and these were shared with other participants as optional cases. Participants were instructed to run at least three of the provided grids for the required cases. This includes the finest grid the participant had resources to compute and two coarser grids. Adaptive results are expected to provide the finest grid results and two coarser intermediate adapted grids. Participants were encouraged to provide their own grids generated with best practices or solution-adaptive methods.

The workshop-provided inviscid AXIE grids are detailed in Table 2. The WS-Mixed grids have a tetrahedral core grid and a prismatic collar grid. Each WS-Mixed prism is replaced with three tetrahedra to form the purely tetrahedral WS-Tet grids. The JWB grids are only provided as tetrahedral inviscid grids as detailed in Table 3. The range of JWB grid sizes is narrower than the other cases. A finer JWB grid was created, but it was generated with a different version of grid generation software and has a slightly different topology and resolution distribution. This finer JWB is provided as an optional grid because of these differences.

Table 2. AXIE required inviscid grid family.

\begin{tabular}{rrrrrr} 
& & \multicolumn{3}{c}{ WS-Mixed } & WS-Tet \\
Scale & Nodes & Tetrahedra & Pyramids & Prisms & Tetrahedra \\
\hline 100 & $56,085,031$ & $60,879,240$ & 0 & $90,419,200$ & $332,136,840$ \\
128 & $15,911,412$ & $13,785,090$ & 0 & $26,655,408$ & $93,751,314$ \\
160 & $5,077,104$ & $3,624,640$ & 0 & $8,686,000$ & $29,682,640$ \\
200 & $1,601,681$ & 932,426 & 0 & $2,770,400$ & $9,243,626$ \\
256 & 646,467 & 220,318 & 0 & $1,161,576$ & $3,705,046$
\end{tabular}

Table 3. JWB required inviscid grid family.

\begin{tabular}{rrr} 
Scale & Nodes & $\begin{array}{c}\text { WS-Tet } \\
\text { Tetrahedra }\end{array}$ \\
\hline 070 & $18,875,613$ & $109,141,197$ \\
083 & $11,335,260$ & $65,432,421$ \\
100 & $6,491,425$ & $37,397,159$
\end{tabular}

The $\mathrm{C} 25 \mathrm{~F}$ and $\mathrm{C} 25 \mathrm{P}$ grids are provided with and without boundary layer resolution to enable viscous and inviscid simulations, see Table 4 and Table 5. The first cell height of the boundary layer grid are designed to have a $y^{+}$ value of one for the 100 scale grids for a flight unit Reynolds of 5.70 million per meter. The inviscid core grids are tetrahedral. The viscous core grids have a prismatic boundary layer that transitions to unstructured tetrahedra with pyramid elements. The collar grids are prisms. The grids are provided as mixed element (WS-Mixed) or converted to tetrahedra (WS-Tet) [67]. 
Table 4. C25F required inviscid and viscous grid families.

\begin{tabular}{rrrrrr}
\multicolumn{7}{c}{ Inviscid } \\
\hline \multicolumn{7}{c}{ WS-Mixed } \\
Scale & Nodes & Tetrahedra & Pyramids & Prisms & WS-Tet \\
Tetrahedra \\
\hline 064 & $103,724,343$ & $161,770,523$ & 0 & $151,236,160$ & $615,479,003$ \\
080 & $51,542,500$ & $82,620,767$ & 0 & $74,194,500$ & $305,204,267$ \\
100 & $26,923,206$ & $42,433,653$ & 0 & $38,890,800$ & $159,106,053$ \\
128 & $13,083,168$ & $20,317,100$ & 0 & $18,921,920$ & $77,082,860$ \\
160 & $6,323,343$ & $10,327,822$ & 0 & $8,918,375$ & $37,082,947$ \\
200 & $3,419,776$ & $5,564,030$ & 0 & $4,810,500$ & $19,995,530$ \\
\multicolumn{5}{c}{ Viscous } \\
\hline \multicolumn{5}{c}{ WS-Mixed } \\
Scale & Nodes & Tetrahedra & Pyramids & Prisms & Tetrahedra \\
\hline 064 & $138,478,889$ & $153,886,434$ & 322,866 & $223,048,965$ & $823,679,061$ \\
080 & $69,833,869$ & $79,008,828$ & 213,368 & $111,723,909$ & $414,607,291$ \\
128 & $17,887,604$ & $19,393,340$ & 77,668 & $28,720,379$ & $216,481,515$ \\
100 & $36,531,052$ & $40,355,827$ & 124,456 & $58,625,592$ & $105,709,813$ \\
160 & $8,927,997$ & $9,967,336$ & 64,398 & $14,153,295$ & $52,556,017$ \\
200 & $4,789,378$ & $5,150,691$ & 45,214 & $7,616,515$ & $28,090,664$
\end{tabular}

Table 5. C25P required inviscid and viscous grid families.

\begin{tabular}{|c|c|c|c|c|c|}
\hline \multicolumn{6}{|c|}{ Inviscid } \\
\hline & & Tetrahedr & WS-Mixed & Prisms & $\begin{array}{l}\text { WS-Tet } \\
\text { Thtrahedra }\end{array}$ \\
\hline $\begin{array}{r}\text { Scale } \\
080\end{array}$ & $\begin{array}{r}\text { Nodes } \\
51873933\end{array}$ & $\begin{array}{l}\text { letrahedra } \\
85700551\end{array}$ & $\begin{array}{r}\text { Pyramias } \\
0\end{array}$ & 73798250 & 307095301 \\
\hline 100 & 27079152 & 44054831 & 0 & 38644800 & 159989231 \\
\hline 128 & 13209858 & 21010609 & 0 & 18935200 & 77816209 \\
\hline 160 & 6393433 & 10768198 & 0 & 8906000 & 37486198 \\
\hline 200 & 3421840 & 5545089 & 0 & 4814200 & 19987689 \\
\hline \multicolumn{6}{|c|}{ Viscous } \\
\hline Scale & Nodes & Tetrahedra & $\begin{array}{l}\text { WS-Mixed } \\
\text { Pyramids }\end{array}$ & Prisms & $\begin{array}{l}\text { WS-Tet } \\
\text { Tetrahedra }\end{array}$ \\
\hline 080 & $70,551,816$ & $82,057,292$ & 202,468 & $112,110,806$ & $418,794,646$ \\
\hline 100 & $36,907,088$ & $42,022,384$ & 114,297 & $58,790,636$ & $218,622,886$ \\
\hline 128 & $18,137,685$ & $20,094,813$ & 71,987 & $28,965,739$ & $107,136,004$ \\
\hline 160 & $9,052,973$ & $10,311,227$ & 53,723 & $14,282,816$ & $53,267,121$ \\
\hline 200 & $4,856,211$ & $5,314,632$ & 41,888 & $7,690,822$ & $28,470,874$ \\
\hline
\end{tabular}




\section{Submissions}

Submissions were collected from the participants with an option to update their submission after the workshop. An extraction macro was provided to the participants with each case description. The participant submissions were corrected to eliminate formatting errors. Participants were contacted for clarification and correction when the extraction location was incorrect, signatures were incomplete, significant differences existed between submissions of the same participant, or boundary conditions were suspect.

The participant-provided submissions are available on the FTP server (with a link from lbpw.larc.nasa. gov) along with derived workshop committee data. This data set includes nearfield signatures, forces, moments, and iterative convergence. Optional loudness and ground signatures are available for verification of the committee analysis when provided. Workshop committee derived ground, loudness, and resampled nearfield signatures at a common 1 $\mathrm{cm}$ resolution. Pointwise mean and standard deviation statistics are available for resampled nearfield signatures and propagated ground signatures. Independent analysis of the data set or reproduction of the data set in participant papers is encouraged.

\section{A. AXIE Submissions}

The submissions for the AXIE model are listed in Table 6. The first letter is the participant identifier from Table 1. The Code used for each case is described in the Participants subsection. The WS-Mixed and WS-Tet grid families are described in the Required Grids section. The characteristic grid length $h$ is computed as the number of control volumes to the negative one-third power. It is scaled to have value of one for a 10 million control volume simulation.

\begin{tabular}{|c|c|c|c|c|}
\hline Case & Code & Physics & Grid & Millions of Control Volumes $(h)$ \\
\hline AA & DLR-TAU-V & Euler & WS-Mixed & $15.9(0.86), 5.1(1.25), 1.6(1.84), 0.6(2.49)$ \\
\hline $\mathrm{AB}$ & DLR-TAU-V & Euler & WS-Tet & $15.9(0.86), 5.1(1.25), 1.6(1.84), 0.6(2.49)$ \\
\hline BA & HUNS3D & Euler & WS-Mixed & $12.3(0.93), 3.7(1.39), 1.4(1.93)$ \\
\hline $\mathrm{BC}$ & HUNS3D & RANS-SA & HUNS3D-Mixed & $24.2(0.75), 11.7(0.95), 6.3(1.17)$ \\
\hline $\mathrm{CA}$ & FUN3D-VA & Euler & WS-Mixed & $56.1(0.56), 15.9(0.86), 5.1(1.25), 1.6(1.84), 0.6(2.49)$ \\
\hline $\mathrm{CC}$ & FUN3D-VA & Euler & WS-Tet & $56.1(0.56), 15.9(0.86), 5.1(1.25), 1.6(1.84), 0.6(2.49)$ \\
\hline DA & JTAS & Euler & WS-Mixed & $56.1(0.56), 15.9(0.86), 5.1(1.25), 1.6(1.84), 0.6(2.49)$ \\
\hline DB & JTAS & Euler & WS-Tet & $56.1(0.56), 15.9(0.86), 5.1(1.25), 1.6(1.84), 0.6(2.49)$ \\
\hline EA & LAVA & RANS-SA & CGT-Overset & $25.0(0.74), 8.0(1.08)$ \\
\hline $\mathrm{EC}$ & LAVA & Euler & STAR-CCM+Poly & $1.8(1.77), 1.3(2.00), 1.1(2.10)$ \\
\hline FA & Cart3D & Euler & Adapt-Cart & $26.0(0.73), 9.0(1.04), 3.2(1.46)$ \\
\hline GA & CartOVER & Euler & Cart+Struct & $240.8(0.35), 126.7(0.43), 67.1(0.53), 34.4(0.66), 19.4(0.80), 9.9(1.00)$ \\
\hline HA & $\mathrm{CFD}++$ & Euler & WS-Mixed & $56.1(0.56), 15.9(0.86), 5.1(1.25), 1.6(1.84)$ \\
\hline IA & USM3D & Euler & WS-Tet & $332.1(0.31), 93.8(0.47), 29.7(0.70), 3.7(1.39)$ \\
\hline IC & USM3D & Euler & PW-Tet & $24.0(0.75), 11.9(0.94), 2.8(1.52)$ \\
\hline JA & FUN3D-VL-HLLC & Euler & WS-Mixed & $56.1(0.56), 15.9(0.86), 5.1(1.25)$ \\
\hline $\mathrm{JC}$ & Cart3D & Euler & Adapt-Cart & $41.4(0.62)$ \\
\hline JD & Cart3D & Euler & Fixed-Cart & $22.6(0.76)$ \\
\hline KA & Wolf & Euler & WS-Tet & $5.1(1.25), 1.6(1.84), 0.6(2.49)$ \\
\hline
\end{tabular}

The nearfield ensemble mean and standard deviation for AXIE fine grid submissions are shown in Fig. 8. Section Signal Ensemble Mean and Standard Deviation describes the process used to calculate the pointwise signal statistics. Only Euler nearfield statistics are examined for this case because there are only 2 RANS submissions. There is an increase in the pointwise standard deviation (denoted as error bars) at five body lengths, Fig. 8c, as compared to one and three body lengths in Fig. 8. The pointwise standard deviation (denoted as error bars) in Fig. 8 is largest at the signal extrema. The impact of reconstruction limiters and other dissipation sources has the greatest impact at the extrema, which increases the variation between the methods.

To examine the increase in the standard deviation at five body lengths, the spatial convergence of the submitted signatures is assessed using the approach described in Section Grid Convergence. Figures 9 and 10 show signatures at three and five body lengths, respectively, computed on the fine grids and plotted with the discretization error estimate. Figures $9 \mathrm{a}$ and 10a show the signature and error estimate obtained by most participants. In this group, the signatures and error regions essentially overplot and case FA is shown as a representative example. Upstream of $\tau=0.7$, the signatures demonstrate excellent grid convergence with only very narrow error spikes at the pressure jumps, which is partially an artifact of the error estimation procedure. Downstream of $\tau=0.7$, the error regions are slightly larger, especially at five body lengths, but still remain small even in the wake $(\tau>1.3)$.

Figures 9b-9f and 10b-10f show signatures that have significantly larger error estimates at three and five body lengths, respectively. Case AB in Fig. 9c has a fine grid signature that is very similar to the one shown in Fig. 9a, but 
with an error estimate that is significantly larger. Cases BA, EC, IC and KA have fine grid signatures significantly different from those shown in Figs. 9a and 10a. Note that cases EC and KA used fine grids that contain roughly a factor of eight and four, respectively, fewer control volumes than the fine workshop grid. Case IC in Fig. 9e is marked "Low Confidence" because its observed order of accuracy converged to a negative number — consequently, the figure only shows scaled differences assuming first-order convergence to indicate the regions of largest differences between the three submitted signatures. Cases DA and DB converged well at three body lengths, but at five body lengths the signature is dominated by spurious oscillations shown in Fig. 10c. The error estimation procedure failed because the level of error in the signature increased as the grid is refined. Consequently, only scaled solution differences are plotted and the error estimate is marked "Low Confidence."

The convergence of PL on the ground with increasing grid refinement is shown in Fig. 11. The characteristic $h$ for each grid is listed in parenthesis in Table 6. Euler methods are depicted in blue, and RANS methods are depicted in red. The convergence of PL with nearfield grid refinement (the propagation method uses a fixed grid) is consistent at one and three body lengths, where all the Euler methods approach a value of $78 \mathrm{~dB}$. Cases DA and DB show an increase in PL on the finest grids at five body lengths. Participant D implicated the collar grid construction as the source of oscillations in the JTAS code solutions. This results in the poor nearfield signature shown in Fig. 10c and divergence in PL. Participant $\mathrm{C}$ indicated that iterative convergence was more difficult to attain on the WS-Mixed and WS-Tet AXIE grid systems than the other model workshop grids. Case AB shows a very linear approach to convergence at five body lengths, which is different than one and three. Cases BA, EC, IC and KA show oscillatory convergence with some offset from the $78 \mathrm{~dB}$ value, which is primarily due to the less accurate nearfield signatures as shown in Fig. 10. The RANS-SA case BC shows a much lower PL than the Euler cases, but the trend is increasing with grid refinement. The other RANS-SA case for the AXIE, EA, is obscured by the more numerous Euler submissions.

Box, whisker, and violin plots are provided for the fine grid Euler AXIE submissions in Fig. 12. The two RANSSA submissions are not numerous enough to produce meaningful statistics; therefore, the RANS plots are omitted. As with the PL grid convergence, the one and three body length locations show consistent descriptive statistics. Cases IC and KA are the two low outliers. The five body length extraction location produced two much louder outliers associated with DA and DB and one lower outlier associated with KA.

The residual convergence was only provided by some participants. Figure 13 shows the minimum residual divided by the maximum residual for each grid. Iterative convergence improved with grid refinement for the AXIE model. Lift, drag, and pitching moment was also collected but not provided here because it would not impact the PL results of an axisymmetric case. 


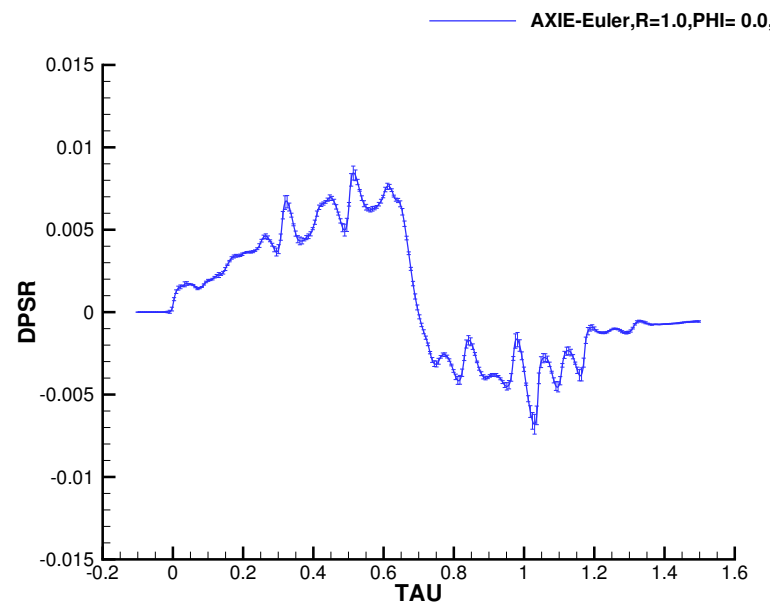

a) $\mathbf{R}=\mathbf{1}$.

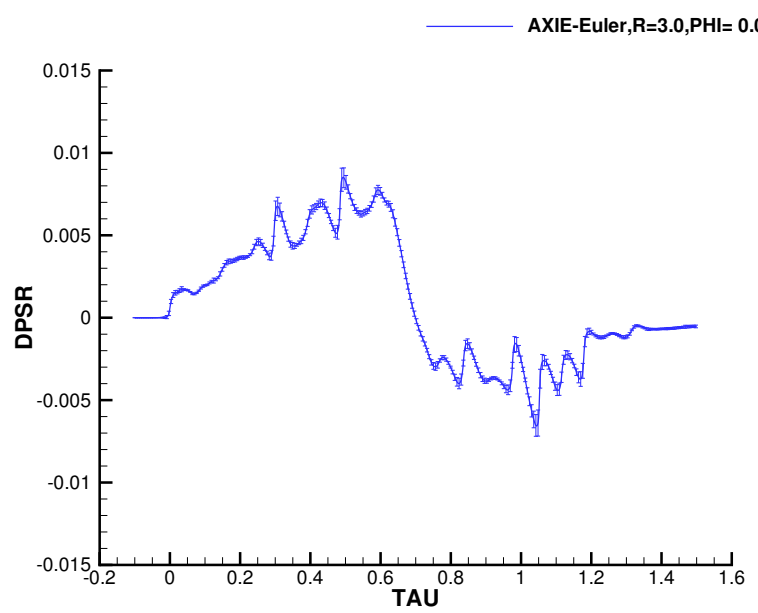

b) $\mathbf{R}=3$.

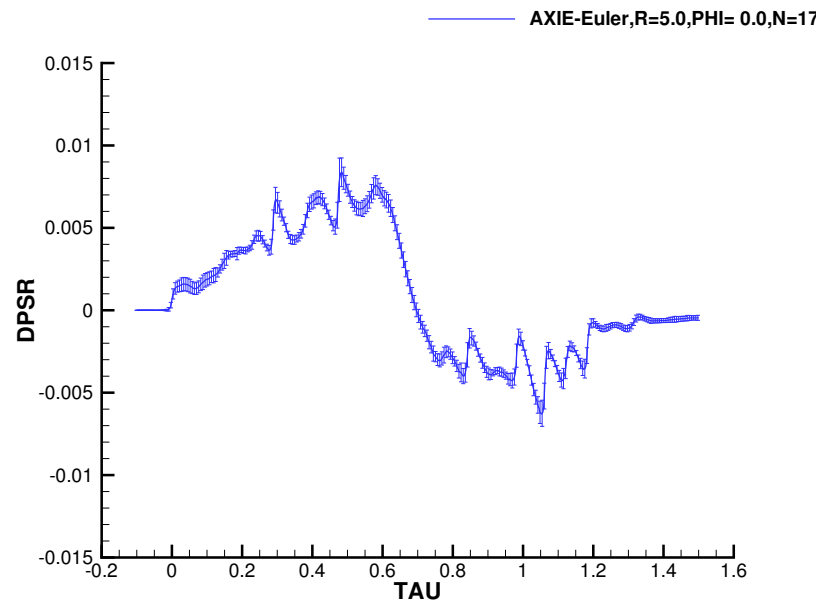

c) $\mathbf{R}=\mathbf{5}$.

Figure 8. AXIE nearfield ensemble mean and standard deviation for fine grids. 


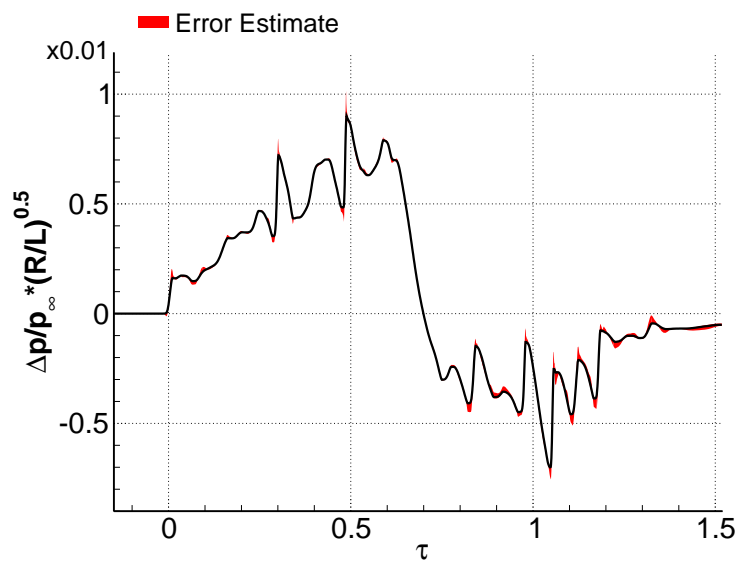

a) AA, CA, CC, DA, DB, FA (shown), GA, HA, IA, JA.

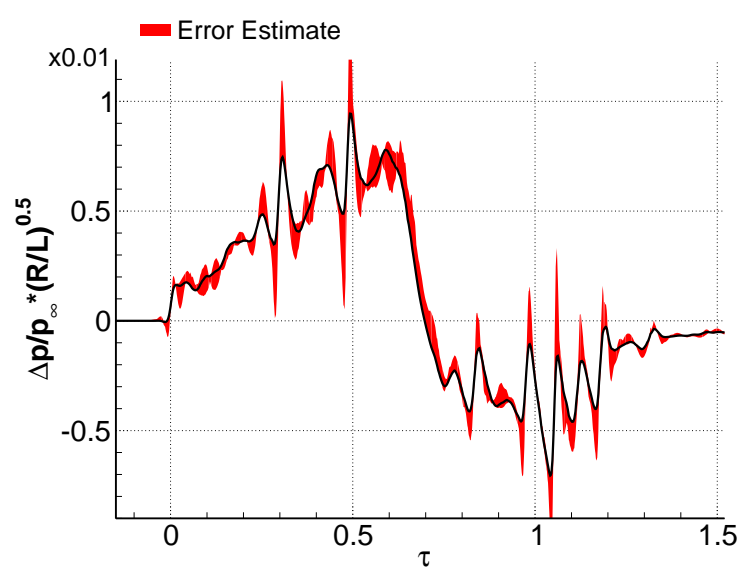

c) $\mathbf{A B}$.

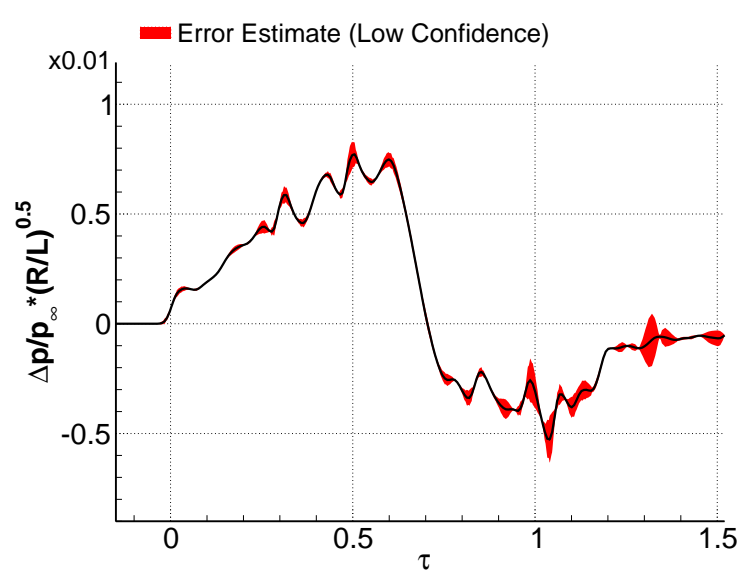

e) IC.

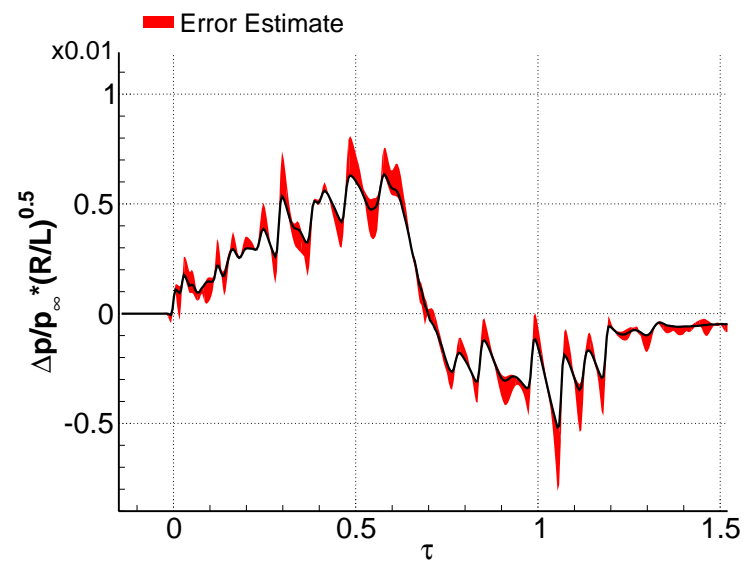

b) BA.

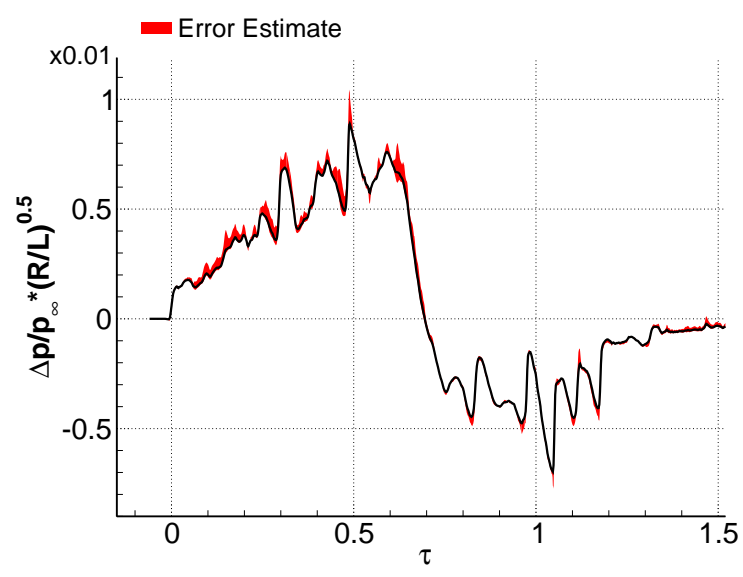

d) EC.

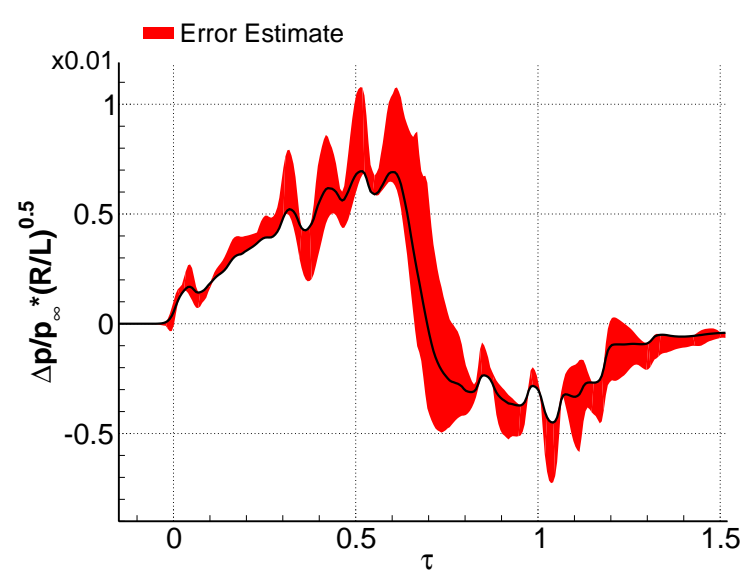

f) KA.

Figure 9. AXIE signature computed on fine grids plotted with discretization error estimates $(\mathbf{R}=\mathbf{3})$. 


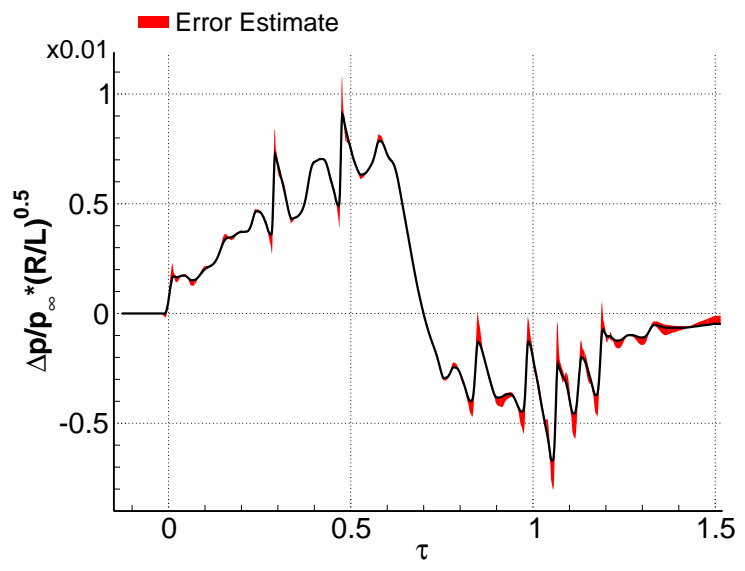

a) AA, CA, CC, FA (shown), GA, HA, IA, JA.

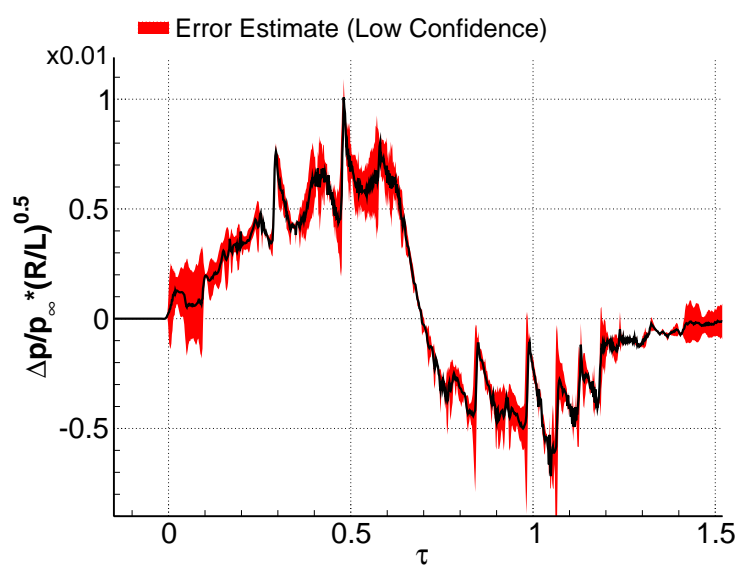

c) DA (shown), DB.

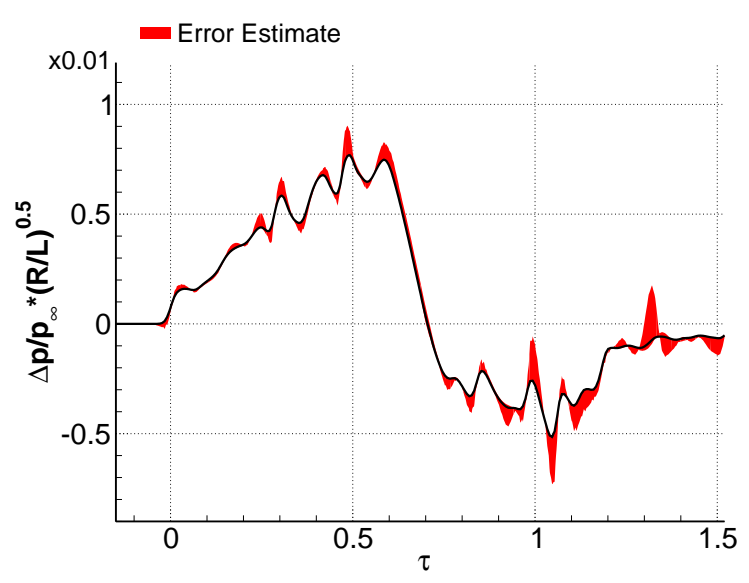

e) IC.

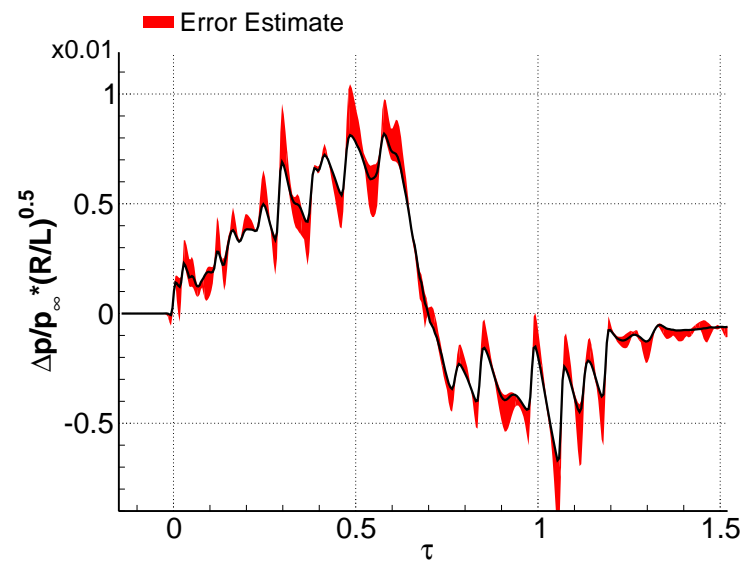

b) $\mathbf{B A}$.

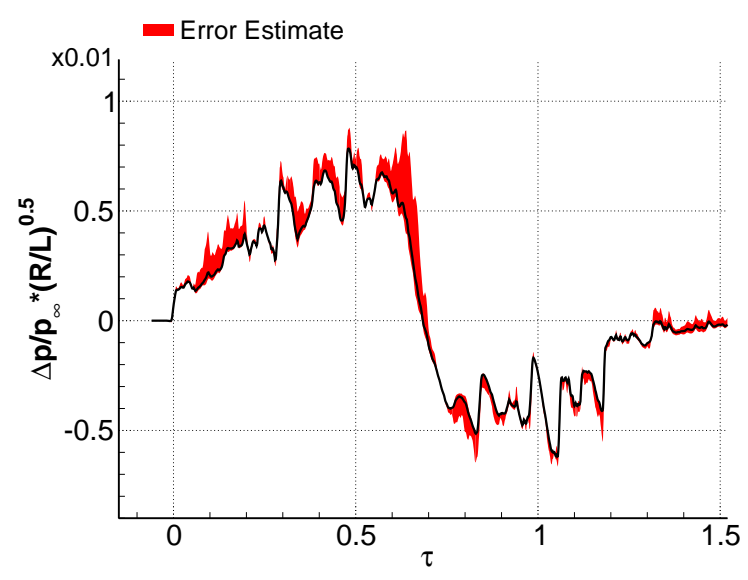

d) $\mathrm{EC}$.

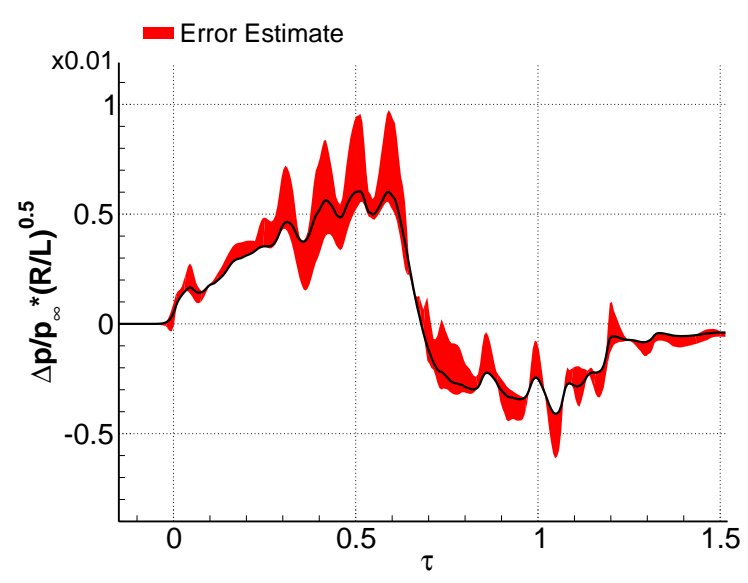

f) KA.

Figure 10. AXIE signature computed on fine grids plotted with discretization error estimates $(\mathbf{R}=\mathbf{5})$. 


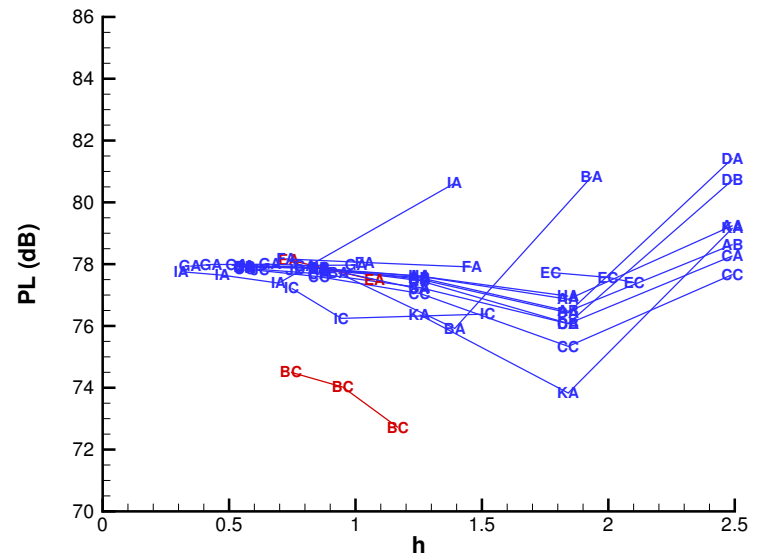

a) $\mathbf{R}=\mathbf{1}$.

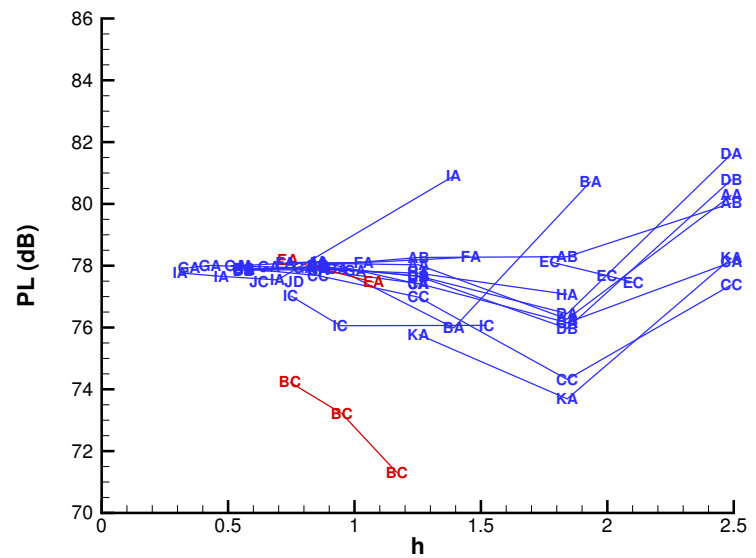

b) $\mathbf{R}=3$.

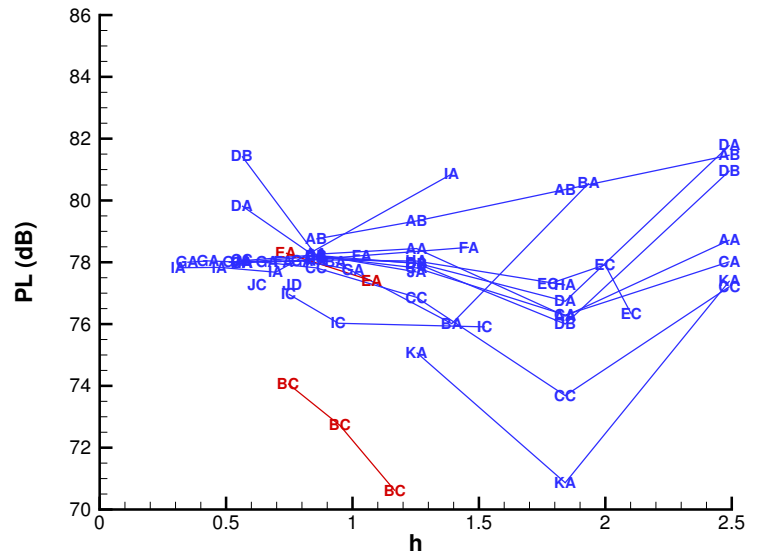

c) $\mathbf{R}=\mathbf{5}$.

Figure 11. AXIE PL grid convergence,-Euler, -RANS. 


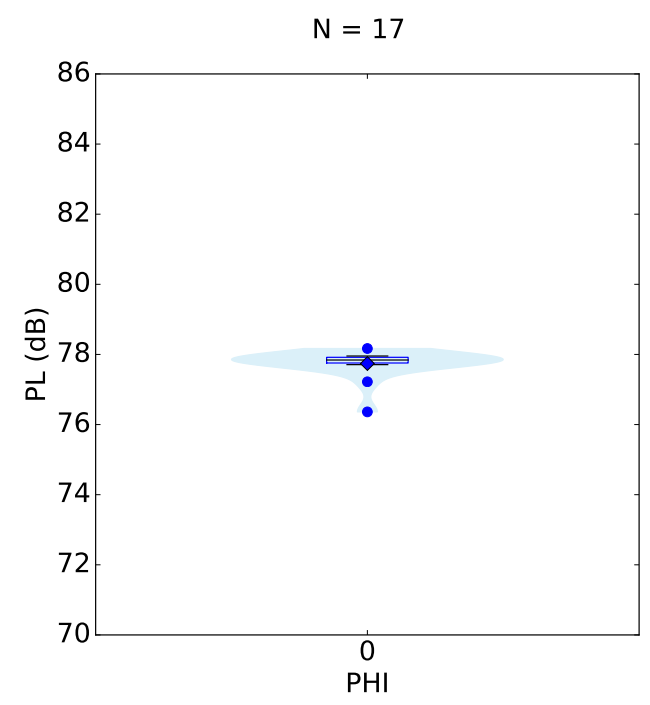

a) R=1. FA high, IC, KA low outliners.

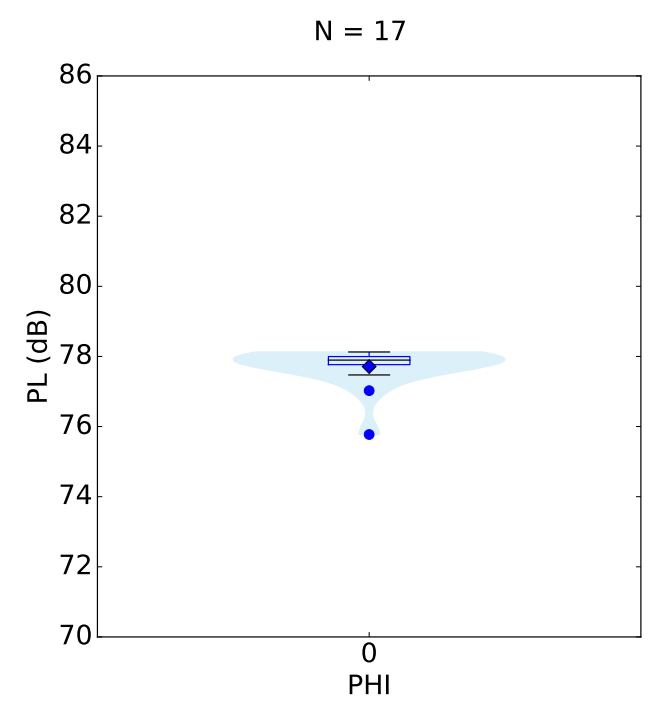

b) $\mathrm{R}=3$. IC, KA low outliners.

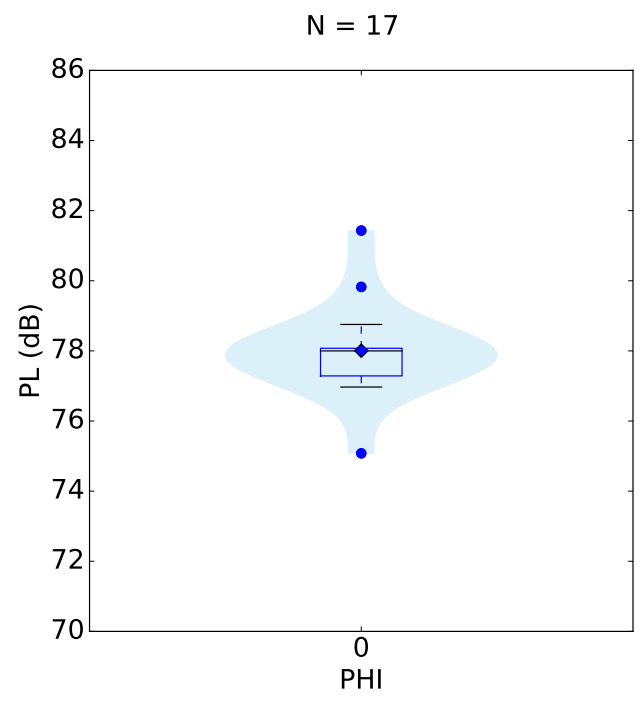

c) $\mathrm{R}=5$. DA, DB high, KA low outlier.

Figure 12. AXIE PL Euler fine grid statistics. 


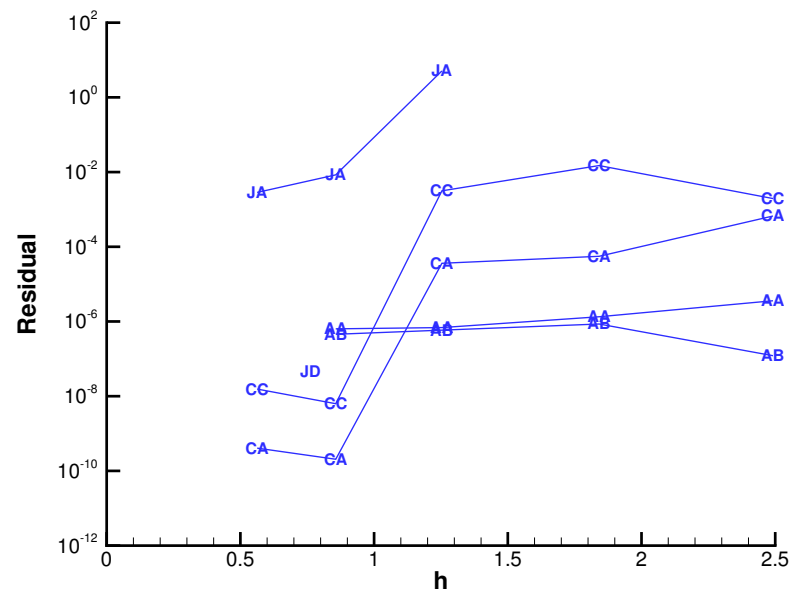

Figure 13. AXIE Residual. 


\section{B. JWB Submissions}

The submissions for the JWB model are listed in Table 7. Only a WS-Tet grid family, as described in the Required Grids section, is available for this model. Cases CC and CD include a fourth finer optional grid that is not a strictly in the same grid family due to grid topology and resolution distribution differences.

Table 7. JWB submissions.

\begin{tabular}{lllll} 
Case & Code & Physics & Grid & Millions of Control Volumes $(h)$ \\
\hline AB & DLR-TAU-V & Euler & WS-Tet & $18.9(0.81), 11.3(0.96), 6.5(1.15)$ \\
AE & DLR-TAU-V & Euler & CENTAUR-Mixed & $29.3(0.70), 15.1(0.87), 8.1(1.07), 1.7(1.80)$ \\
BB & HUNSD & RANS-SA & HUNS3D-Mixed & $40.6(0.63), 21.2(0.78), 12.8(0.92)$ \\
CC & FUN3D-VA & Euler & WS-Tet & $51.4(0.58), 18.9(0.81), 11.3(0.96), 6.5(1.15)$ \\
CD & FUN3D & Euler & WS-Tet & $51.4(0.58), 18.9(0.81), 11.3(0.96), 6.5(1.15)$ \\
DB & JTAS & Euler & WS-Tet & $18.9(0.81), 11.3(0.96), 6.5(1.15)$ \\
EA & LAVA-HWCNS & RANS-SA & CGT-Overset & $32.6(0.67), 18.0(0.82), 10.5(0.98)$ \\
EB & LAVA-Roe & RANS-SA & CGT-Overset & $32.6(0.67), 18.0(0.82), 10.5(0.98)$ \\
EC & LAVA & Euler & STAR-CCM+Poly & $8.8(1.04), 4.0(1.36), 2.1(1.69)$ \\
FA & Cart3D & Euler & Adapt-Cart & $31.7(0.68), 8.9(1.04), 5.9(1.19)$ \\
GA & CartOVER & Euler & Cart+Struct & $233.7(0.35), 123.7(0.43), 64.5(0.54), 34.7(0.66), 19.1(0.81), 10.9(0.97)$ \\
GD & FUN3D-VL & Euler & WS-Tet & $18.9(0.81), 11.3(0.96), 6.5(1.15)$ \\
HB & CFD++ & Euler & WS-Tet & $18.9(0.81), 11.3(0.96), 6.5(1.15)$ \\
IA & USM3D & Euler & WS-Tet & $109.1(0.45), 65.4(0.53), 37.4(0.64)$ \\
JB & FUN3D-VL-HLLC & Euler & WS-Tet & $18.9(0.81), 11.3(0.96), 6.5(1.15)$ \\
JC & Cart3D & Euler & Adapt-Cart & $46.4(0.60)$ \\
JD & Cart3D & Euler & Fixed-Cart & $28.8(0.70)$
\end{tabular}

The nearfield ensemble mean and standard deviation are shown in Fig. 14 near the centerline and Fig. 15 at larger offtrack angles. There are only three fine grid RANS submissions, which is not a large enough sample size to compute robust statistics. With this sample size caveat, the RANS submissions are included because the difference between the Euler and RANS means near the centerline is larger than the standard deviation of either physics group. The strong lower body closeout expansion seen in the Fig. 6 Euler solution on the symmetry plane may be modeled differently by RANS. The difference between the Euler and RANS means reduces with offtrack angle. The magnitude of the difference between Euler and RANS means is generally smaller than the standard deviation of either group in Fig. 15.

Figure 16 shows representative nearfield signatures obtained from inviscid simulations at $R=3$. The inviscid results can be divided into two groups: those participants who used the workshop-provided tetrahedral grids (WS-Tet), and those who did not. The signatures and error estimates computed for the WS-Tet grids are shown in Figs. 16c (ontrack) and $16 \mathrm{~d}\left(\mathrm{PHI}=50^{\circ}\right)$, while those obtained for the custom mixed-element, adapted, or structured grids are shown in Figs. 16a (on-track) and $16 \mathrm{~b}\left(\mathrm{PHI}=50^{\circ}\right)$. Signatures AE, EC, FA, and GA essentially overplot, with only small differences primarily in the wake region $(\tau>1.2)$. Case GA is plotted as a representative example. The numerous shocks in this signature are well resolved, and the error estimate is small. There is a greater variety in the signatures computed on the WS-Tet grids, especially in the amplitude of the strong shock near $\tau=1.1$. We show signature CC as an example. The signatures in this group are poorly resolved and are well outside the asymptotic range; the error analysis indicates that the level of error in the signature is increasing as the mesh is refined. Consequently, only scaled solution differences are plotted, and the error estimate is marked "Low Confidence" in Figs. 16c and 16d.

Figure 17 shows selected nearfield signatures obtained from viscous simulations at $\mathrm{R}=3$. Case BB (Figs. 17a and 17b) is poorly resolved, with most features of the signature highly dissipated. Cases EA and EB are both well resolved (Figs. 17c-17f). These cases use the same structured (overset) grid, but case EA uses a fifth-order accurate discretization of the convective terms while EB uses a second-order discretization. Case EA predicts sharper pressure jumps. The observed order of accuracy is similar for $\mathrm{EA}$ and $\mathrm{EB}$ for the on-track signature. At $\mathrm{PHI}=50^{\circ}$, the observed order approximately doubles for EA relative to EB.

The convergence of PL with grid refinement is shown in Fig. 18 near the centerline and Fig. 19 at larger offtrack angles. The Euler submissions are shown in blue and the three RANS submissions are shown in red. The Euler and RANS physical models appear to be approaching different PL values. This confirms the observation in the nearfield ensemble means where the differences were greatest on the centerline and reduced with offtrack angle. The grid convergence trends are less convincing than the AXIE model. The spread in the Euler submissions is surprisingly small despite the relatively large differences in the nearfield signatures shown in Fig. 16. The spread in the RANS results is due to the large differences discussed in Fig. 17 between participants B and E.

Box, whisker, and violin plots of PL calculated from the fine grid Euler submissions are shown in Fig. 20. The PL values are plotted for each offtrack angle to produce PL carpet plots with submissions provided at one and three body lengths. The three RANS submissions would not create meaningful ensemble statistics and are omitted. There 
is a strong dependency on extraction location, which could be due to the spanwise distribution of pressure disturbance of this winged model. The interquartile range (box extent) is larger for the JWB than the AXIE except at PHI $=50^{\circ}$. Outliers tended to be low values. The outliers are the fine grid submissions of cases IA, JC, and JD. Without the optional grid, case CC would also be an outlier.

The convergence of lift and relative iterative convergence for the JWB submissions is shown in Fig. 21. The Euler submissions trend toward a higher lift value than the RANS submissions. Case FA has the lowest lift value of the Euler submissions. The other Euler cases tend to show an increasing lift trend with grid refinement. The relative meanflow equation iterative convergence is shown in blue solid lines and the turbulence model relative convergence is shown in red with dashed lines. 


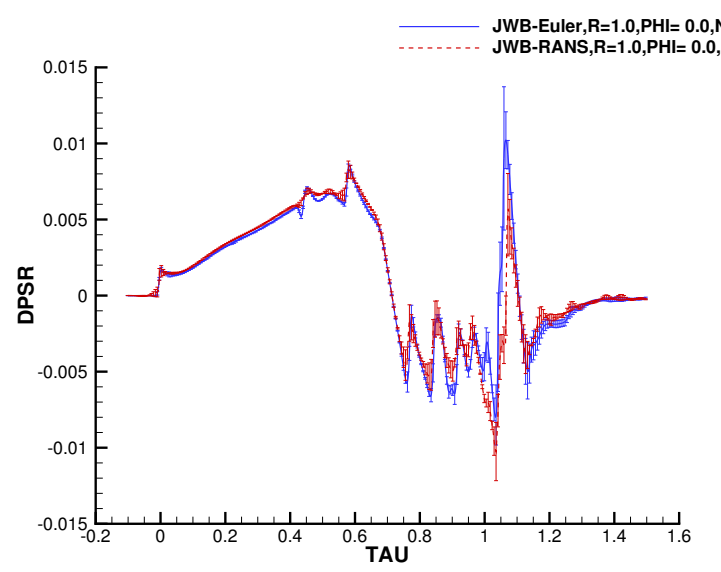

a) $\mathrm{R}=\mathbf{1}, \mathbf{P H I}=\mathbf{0}^{\circ}$.

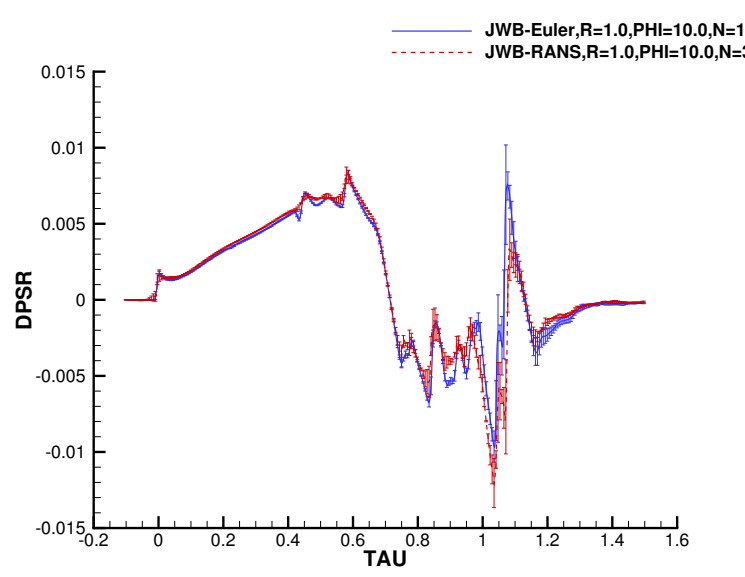

c) $\mathrm{R}=1, \mathrm{PHI}=10^{\circ}$.

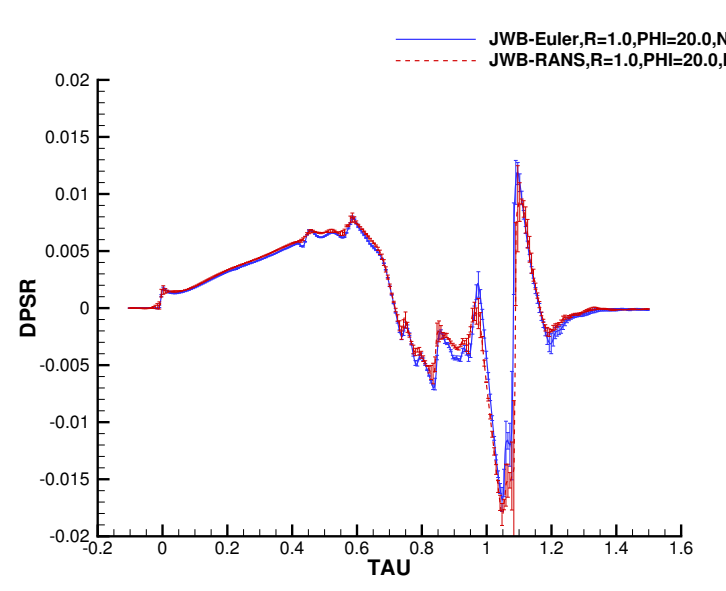

e) $\mathrm{R}=\mathbf{1}, \mathrm{PHI}=\mathbf{2 0}{ }^{\circ}$.

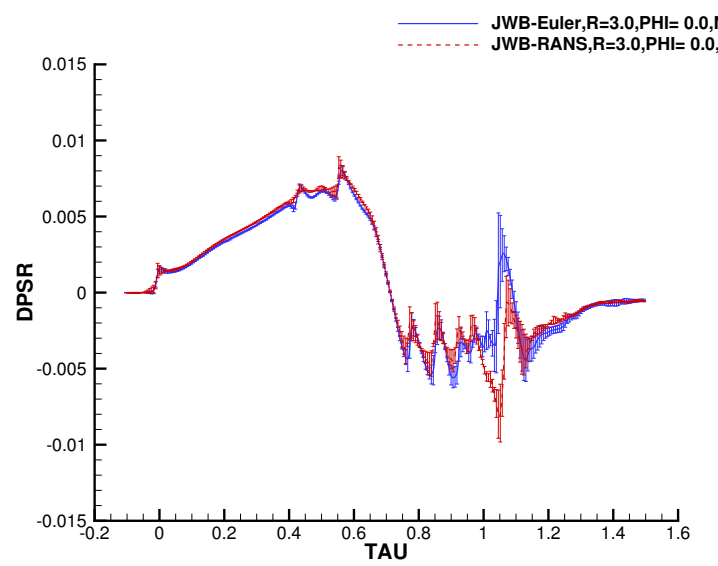

b) $\mathrm{R}=3, \mathrm{PHI}=0^{\circ}$.

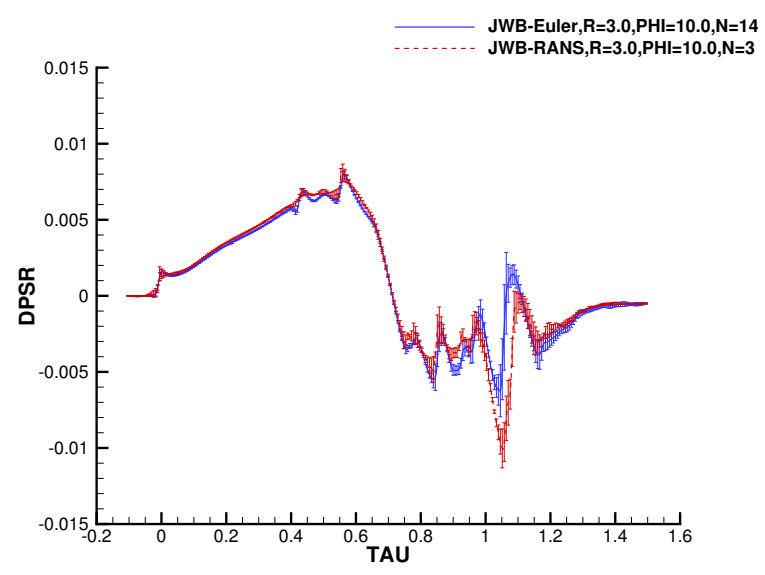

d) $\mathrm{R}=3, \mathrm{PHI}=10^{\circ}$.

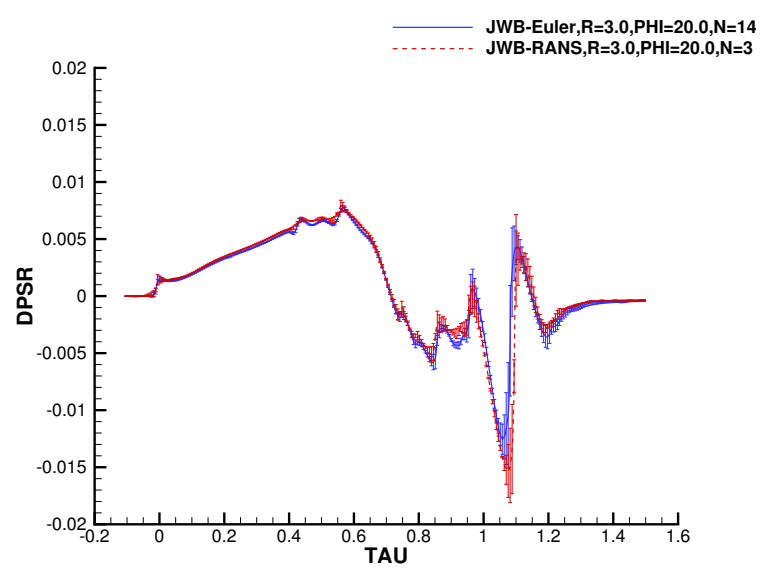

f) $\mathbf{R}=\mathbf{3}, \mathbf{P H I}=\mathbf{2 0}{ }^{\circ}$.

Figure 14. JWB nearfield ensemble mean and standard deviation for fine grids, $\mathrm{PHI}=\mathbf{0}^{\circ}-20^{\circ}$. 


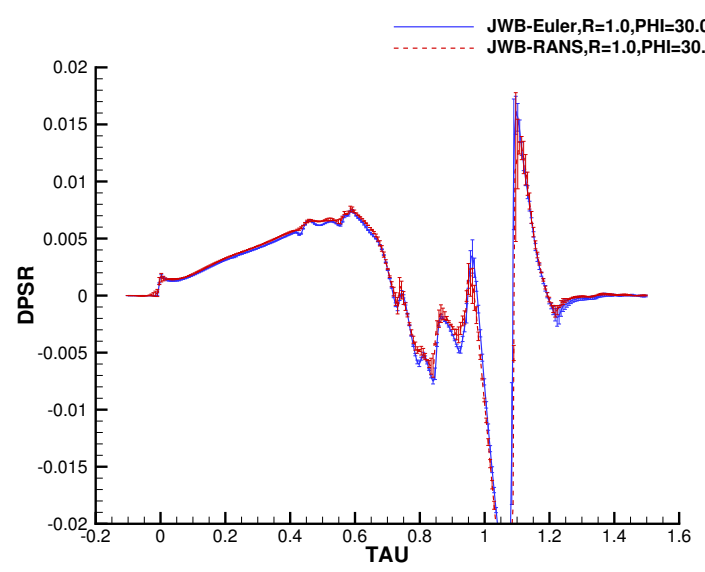

a) $\mathrm{R}=1, \mathrm{PHI}=30^{\circ}$.

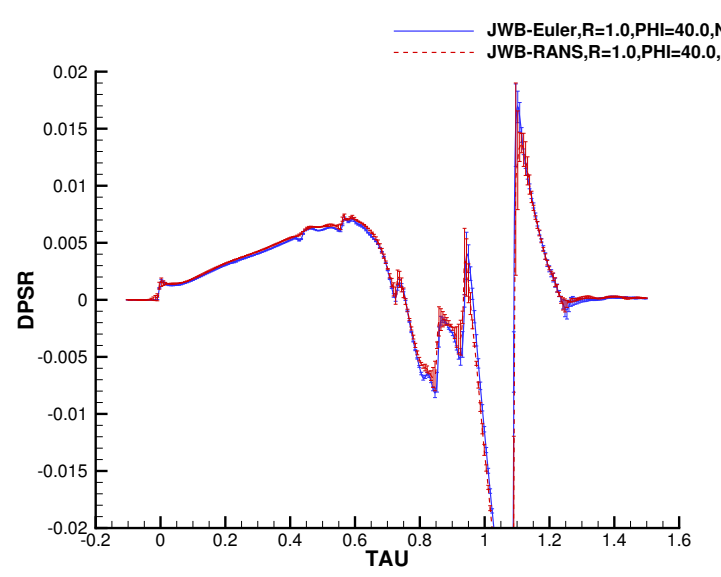

c) $\mathrm{R}=\mathbf{1}, \mathrm{PHI}=\mathbf{4 0 ^ { \circ }}$.

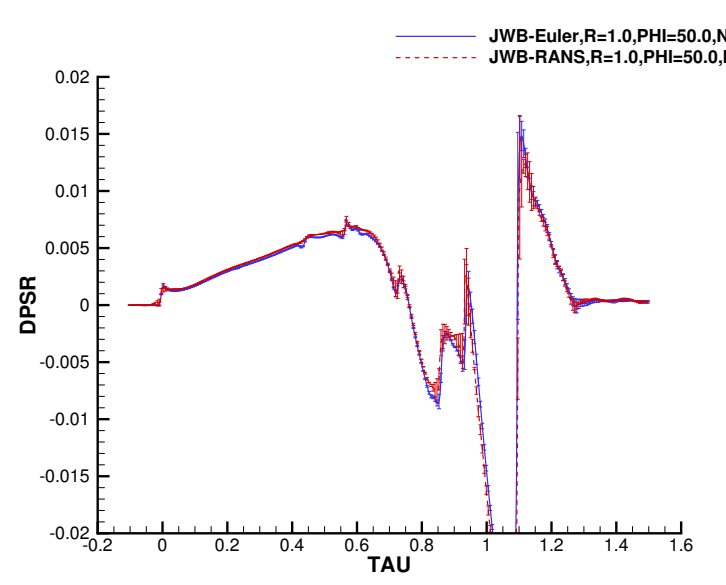

e) $\mathrm{R}=\mathbf{1}, \mathrm{PHI}=\mathbf{5 0}{ }^{\circ}$.

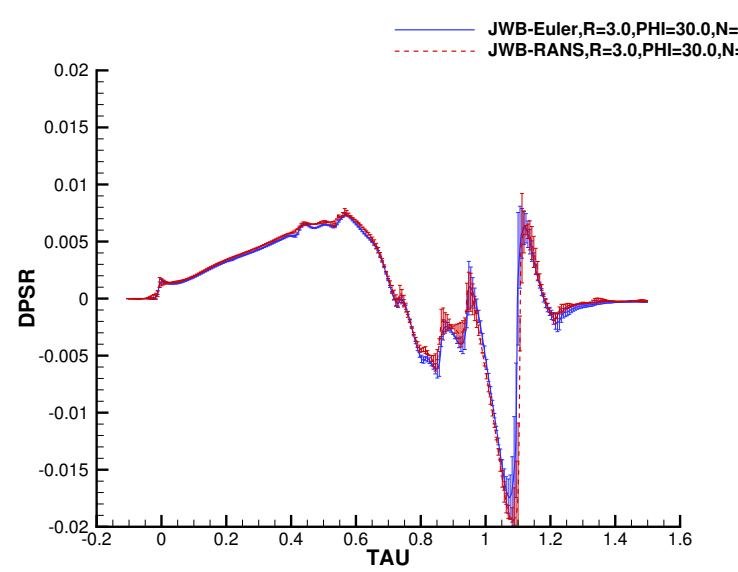

b) $\mathbf{R}=3, \mathrm{PHI}=30^{\circ}$.

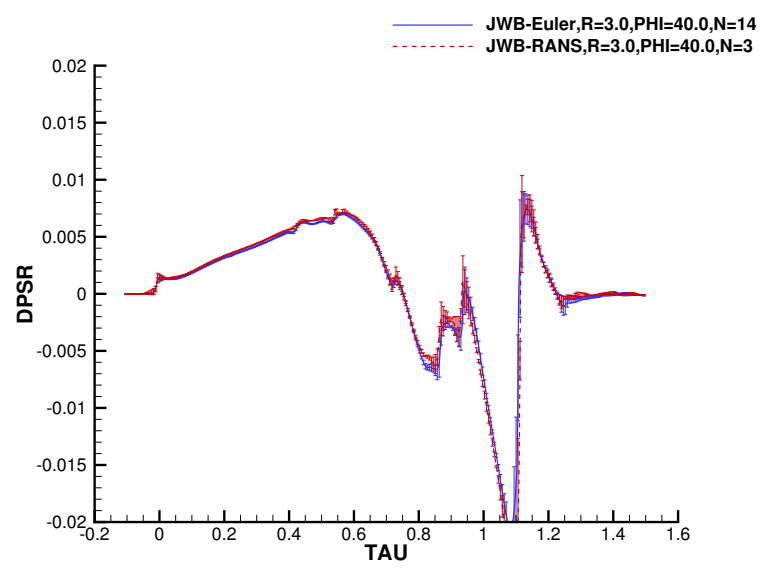

d) $\mathrm{R}=3, \mathrm{PHI}=40^{\circ}$.

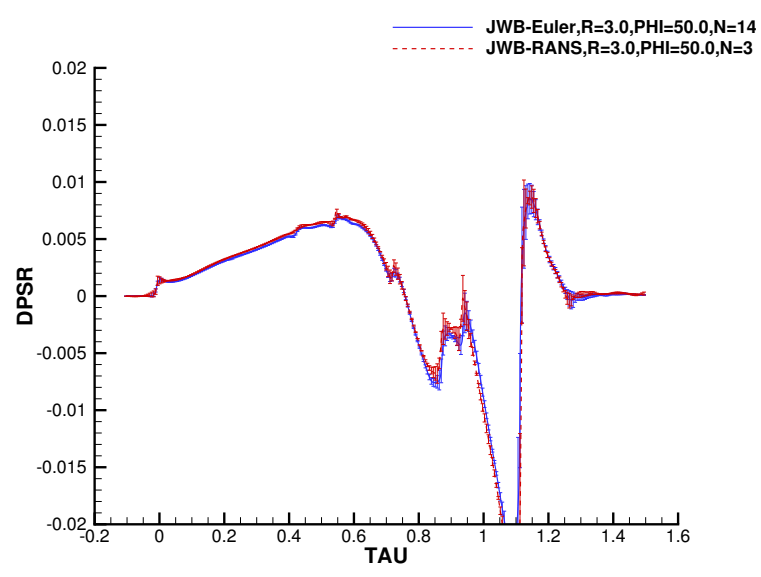

f) $\mathrm{R}=3, \mathrm{PHI}=50^{\circ}$.

Figure 15. JWB nearfield ensemble mean and standard deviation for fine grids, $\mathrm{PHI}=\mathbf{3 0}{ }^{\circ}-\mathbf{5 0}{ }^{\circ}$. 


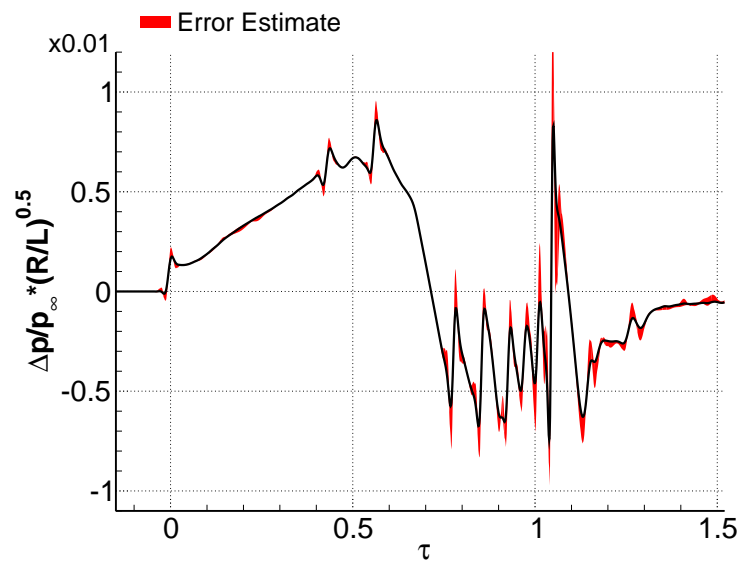

a) $\mathrm{PHI}=0^{\circ}$ : AE, EC, FA, GA (shown).

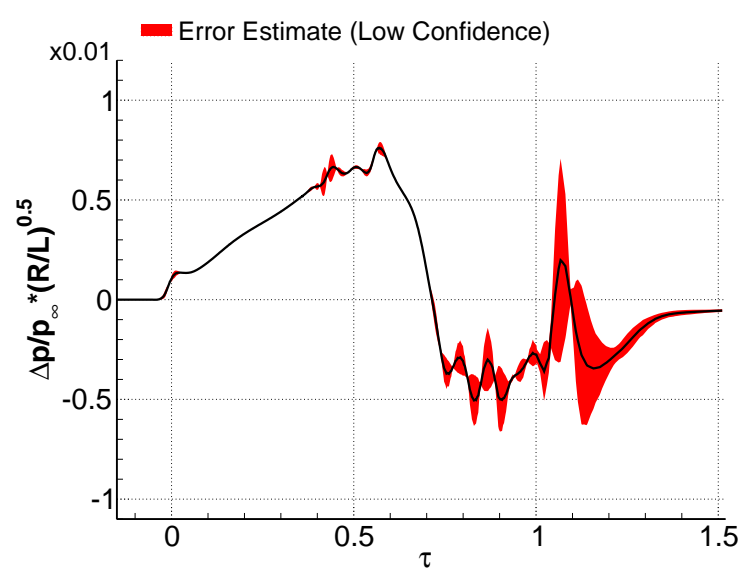

c) $\mathrm{PHI}=0^{\circ}: \mathrm{AB}, \mathrm{CC}($ shown $), \mathrm{CD}, \mathrm{DB}, \mathrm{GD}, \mathrm{HB}, \mathrm{IA}, \mathrm{JB}$.

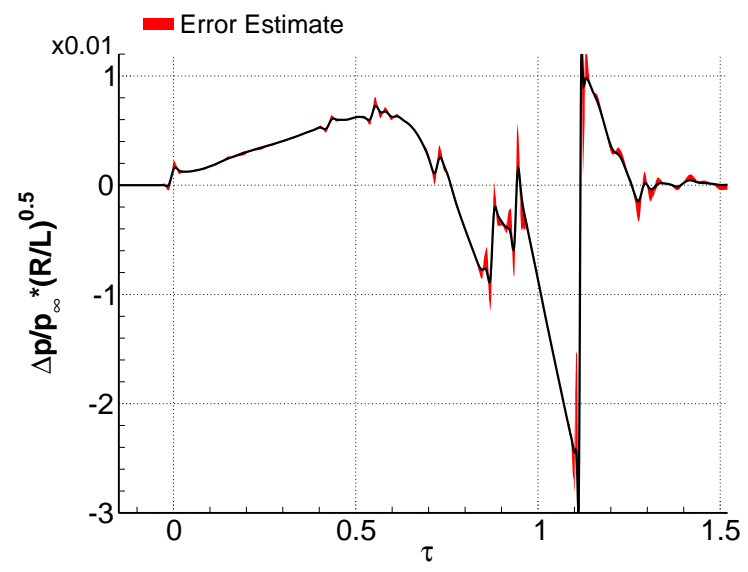

b) $\mathrm{PHI}=50^{\circ}$ : AE, EC, FA, GA (shown).

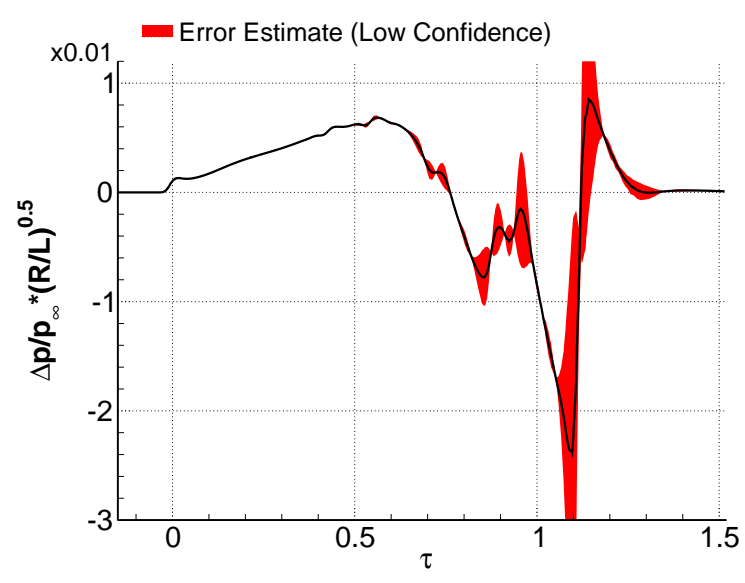

d) $\mathrm{PHI}=50^{\circ}: \mathrm{AB}, \mathrm{CC}$ (shown), CD, DB, GD, HB, IA, JB.

Figure 16. JWB Euler nearfield signatures computed on fine grids with discretization error estimates: $R=3, \mathrm{PHI}=0^{\circ}$ and $50^{\circ}$. 


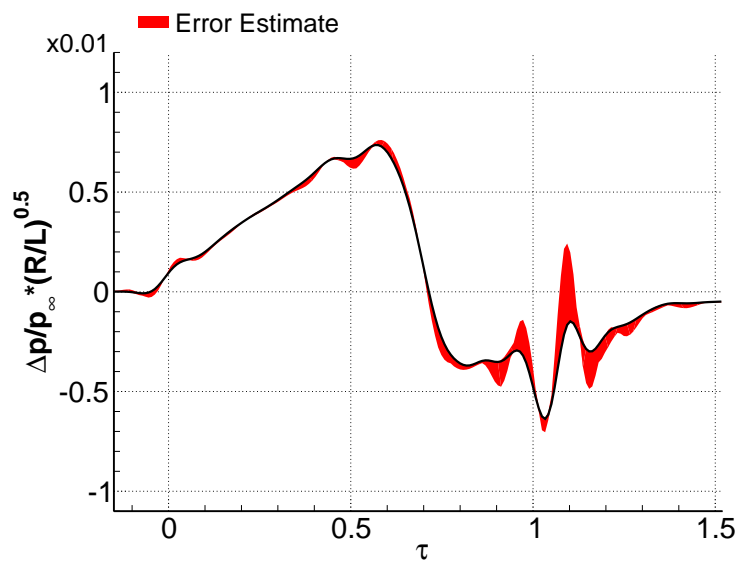

a) $\mathrm{PHI}=0^{\circ}: \mathbf{B B}$.

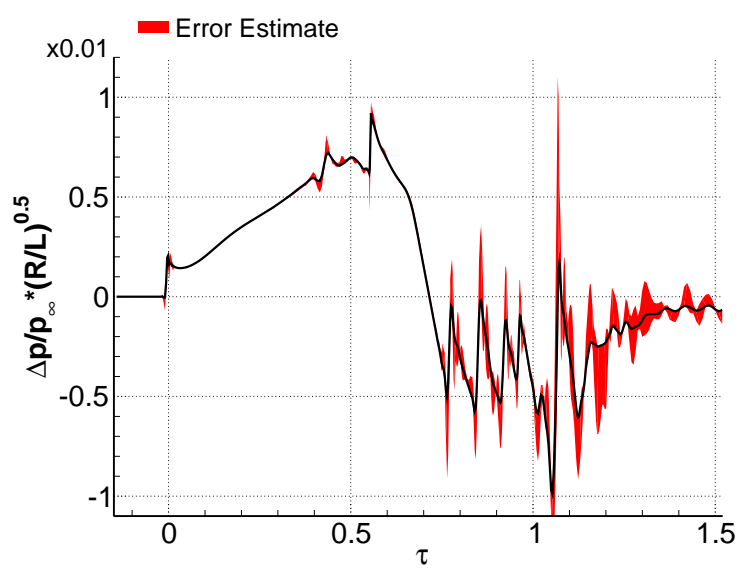

c) $\mathrm{PHI}=0^{\circ}:$ EA.

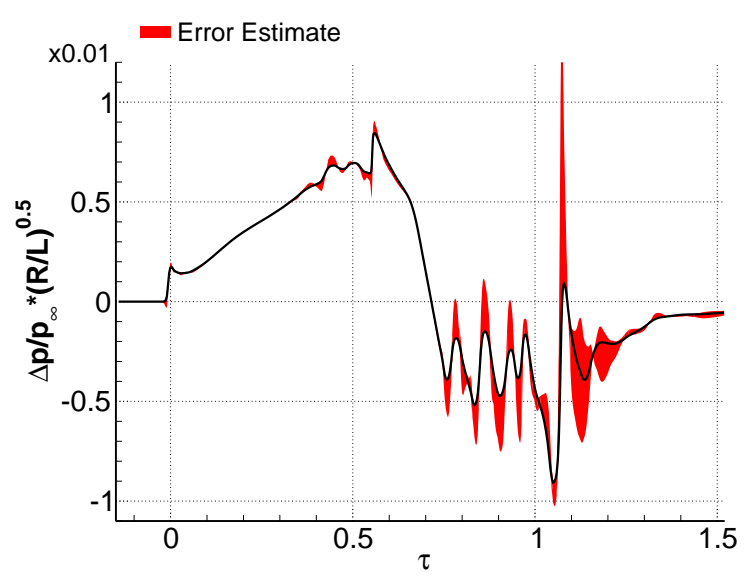

e) $\mathrm{PHI}=0^{\circ}: \mathbf{E B}$.

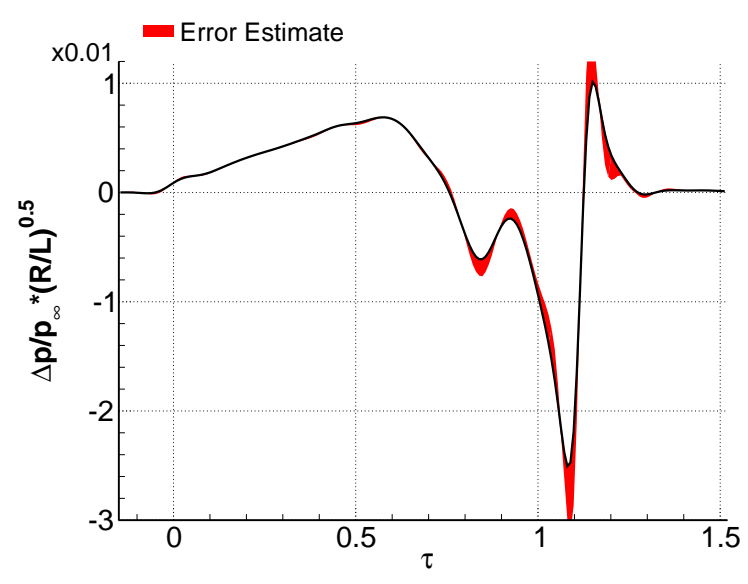

b) $\mathrm{PHI}=50^{\circ}: \mathrm{BB}$.

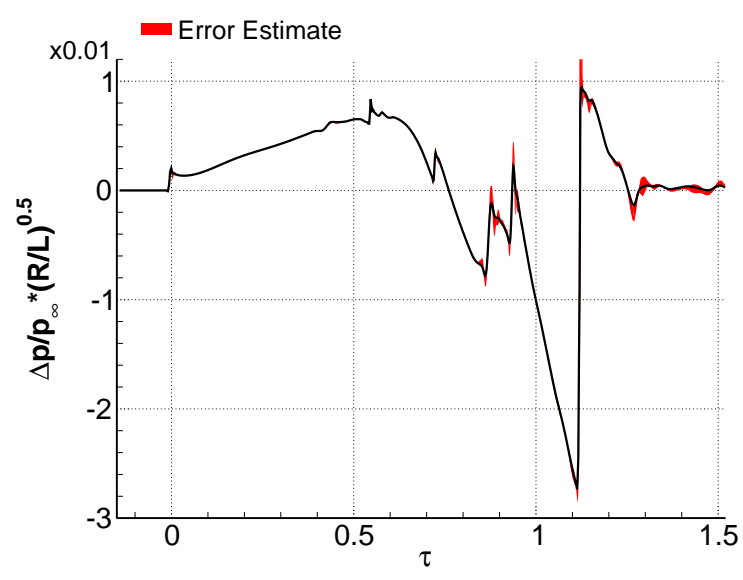

d) $\mathrm{PHI}=50^{\circ}$ : EA.

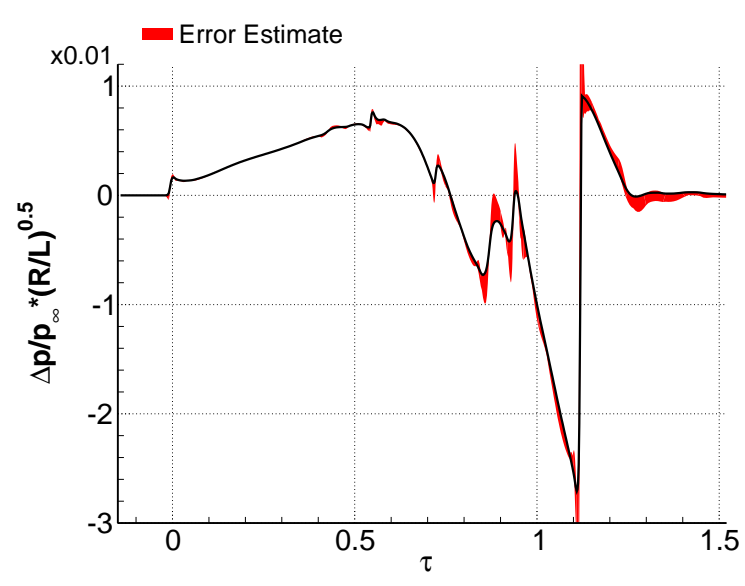

f) $\mathrm{PHI}=50^{\circ}: \mathbf{E B}$.

Figure 17. JWB RANS nearfield signatures computed on fine grids with discretization error estimates: $\mathrm{R}=3$, $\mathrm{PHI}=0^{\circ}$ and $50^{\circ}$. 


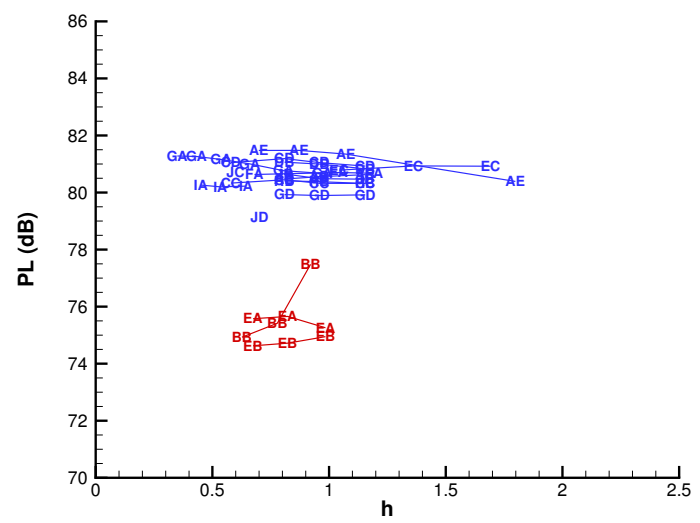

a) $\mathrm{R}=\mathbf{1}, \mathrm{PHI}=\mathbf{0}^{\circ}$.

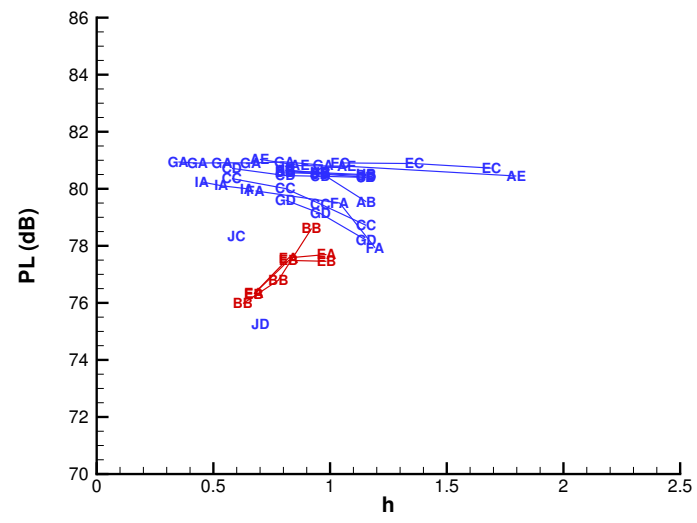

c) $\mathrm{R}=1, \mathrm{PHI}=10^{\circ}$.

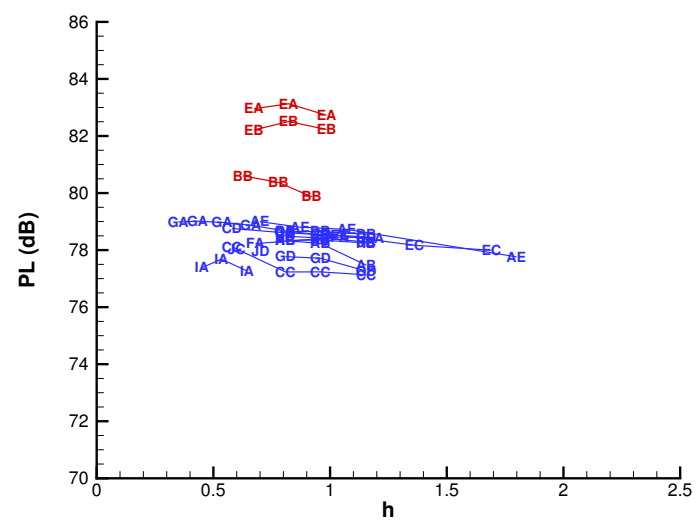

e) $\mathbf{R}=1, \mathrm{PHI}=20^{\circ}$.

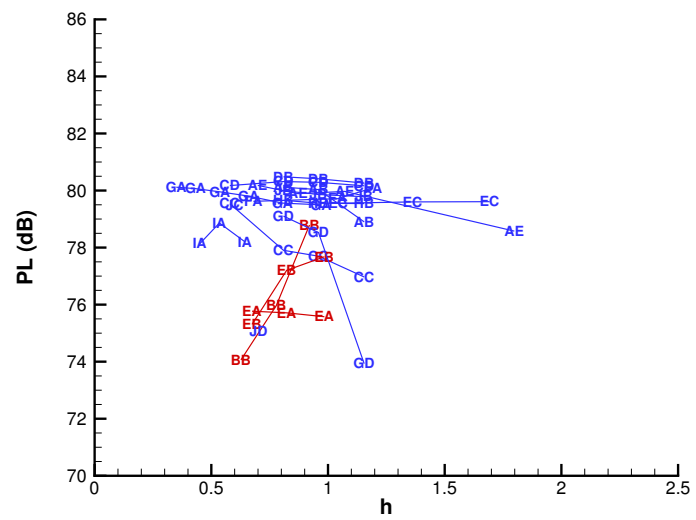

b) $\mathrm{R}=3, \mathrm{PHI}=\mathbf{0}^{\circ}$.

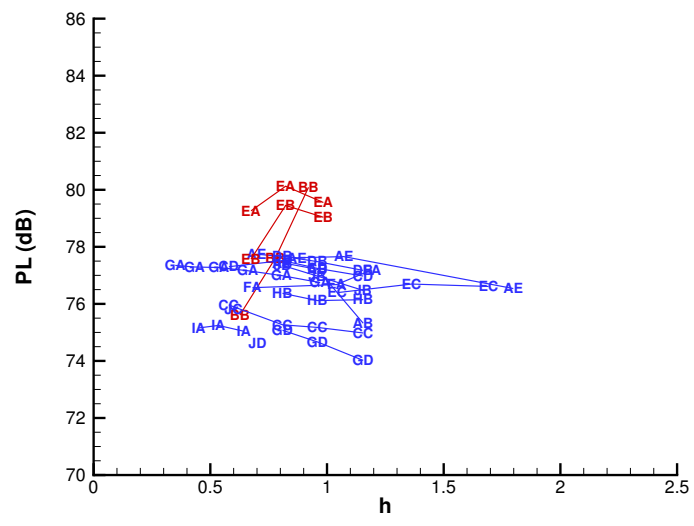

d) $\mathrm{R}=3, \mathrm{PHI}=10^{\circ}$.

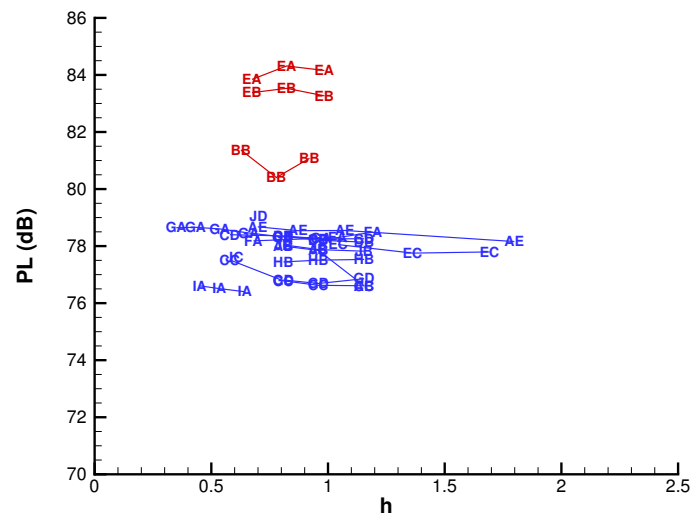

f) $\mathbf{R}=3, \mathbf{P H I}=20^{\circ}$.

Figure 18. JWB PL grid convergence, $\mathrm{PHI}=0^{\circ}-20^{\circ},-$ Euler, - RANS. 


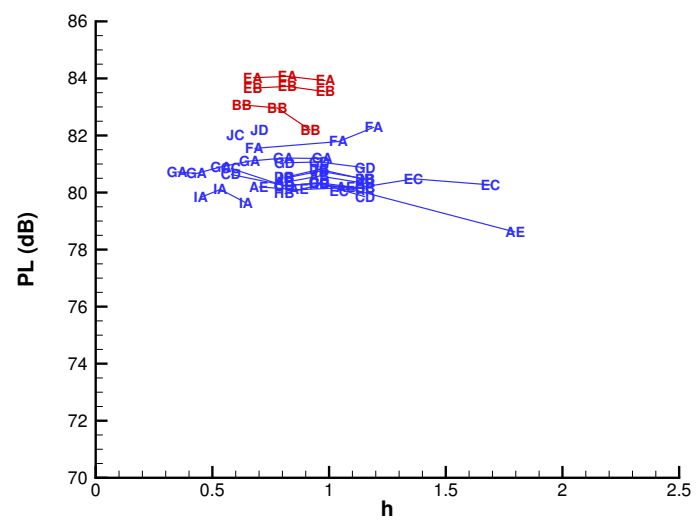

a) $\mathrm{R}=1, \mathrm{PHI}=30^{\circ}$.

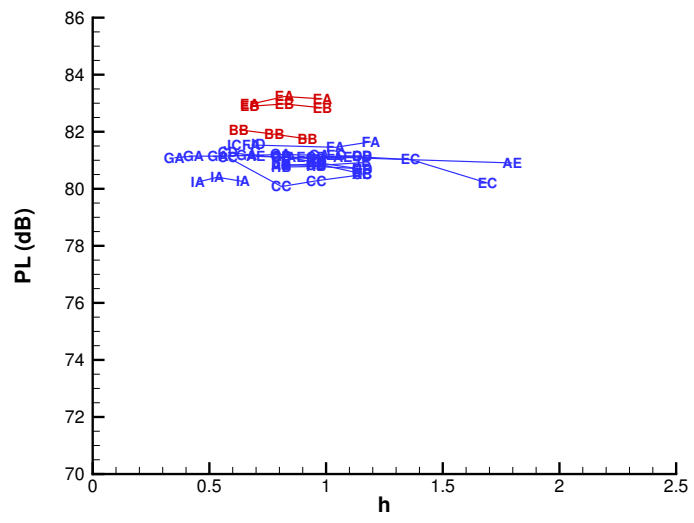

c) $\mathrm{R}=\mathbf{1}, \mathrm{PHI}=\mathbf{4 0 ^ { \circ }}$.

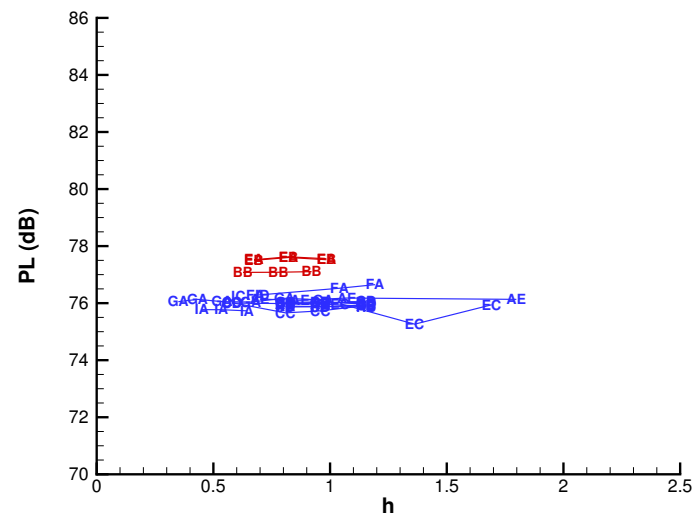

e) $\mathrm{R}=1, \mathrm{PHI}=50^{\circ}$.

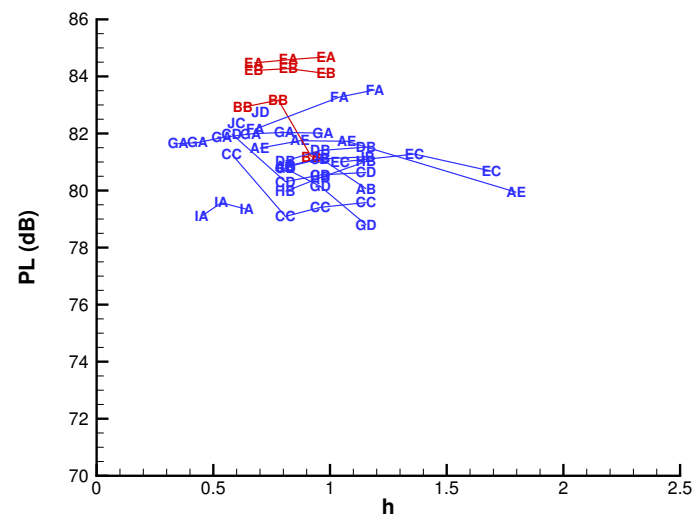

b) $\mathbf{R}=3, \mathrm{PHI}=30^{\circ}$.

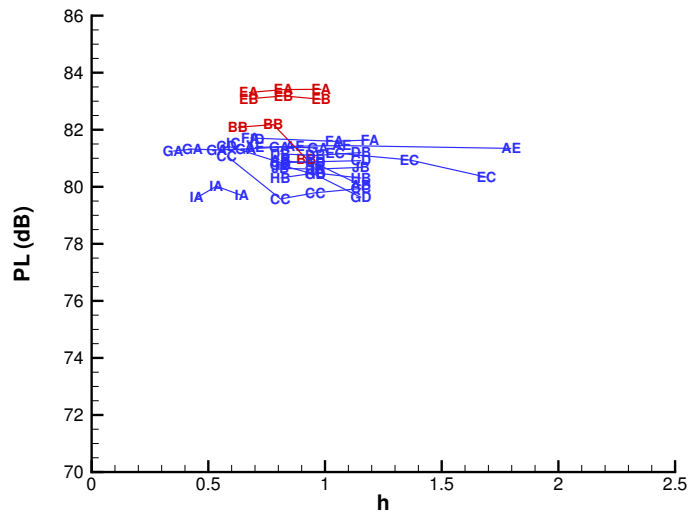

d) $\mathrm{R}=3, \mathrm{PHI}=40^{\circ}$.

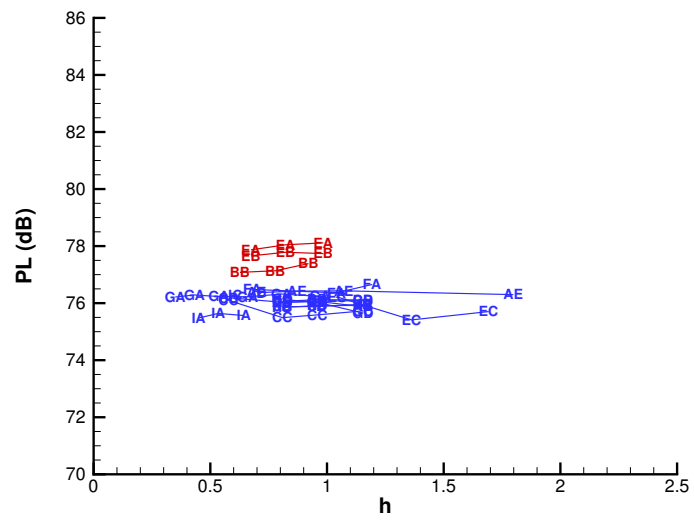

f) $\mathrm{R}=3, \mathrm{PHI}=50^{\circ}$.

Figure 19. JWB PL grid convergence, $\mathrm{PHI}=\mathbf{3 0}^{\circ}-\mathbf{5 0} 0^{\circ}$, -Euler, - RANS. 


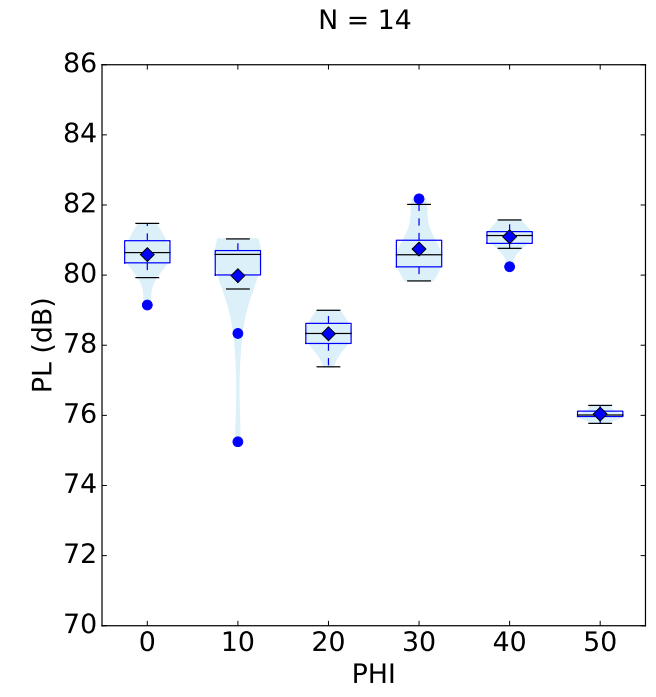

a) Euler R=1. PHI $=10^{\circ}$ : JD low. $\mathrm{PHI}=20^{\circ}$ : JC, JD low. $\mathrm{PHI}=30^{\circ}: \mathrm{JC}, \mathrm{JD}$ high. $\mathrm{PHI}=40^{\circ}$ : IA low.

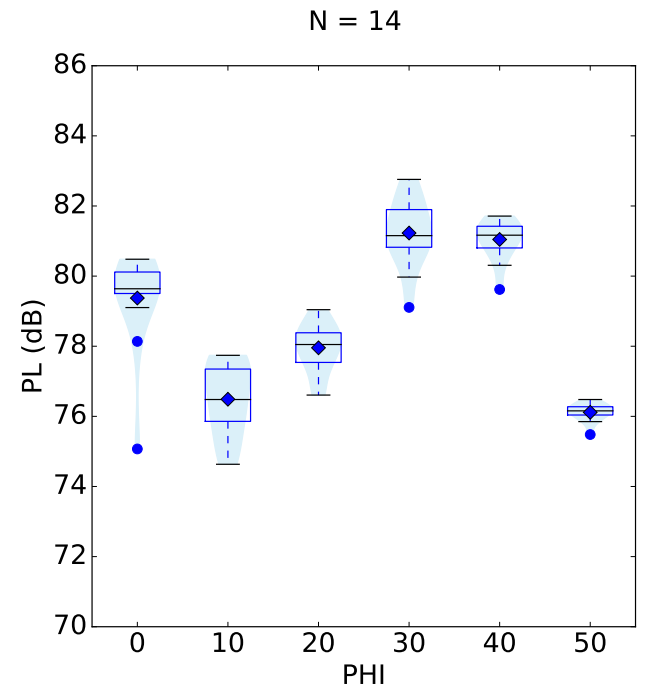

b) Euler $\mathrm{R}=3$. $\mathrm{PHI}=0^{\circ}$ : IA, JD low. $\mathrm{PHI}=\mathbf{3 0}^{\circ}, \mathbf{4 0}^{\circ}, \mathbf{5 0}^{\circ}$ : IA low.

Figure 20. JWB PL Euler fine grid statistics. 


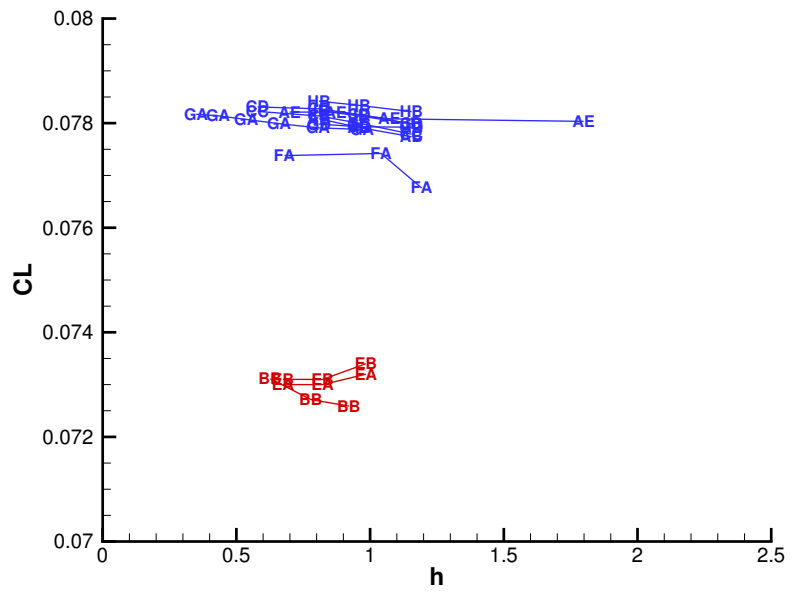

a) Lift Coefficient.

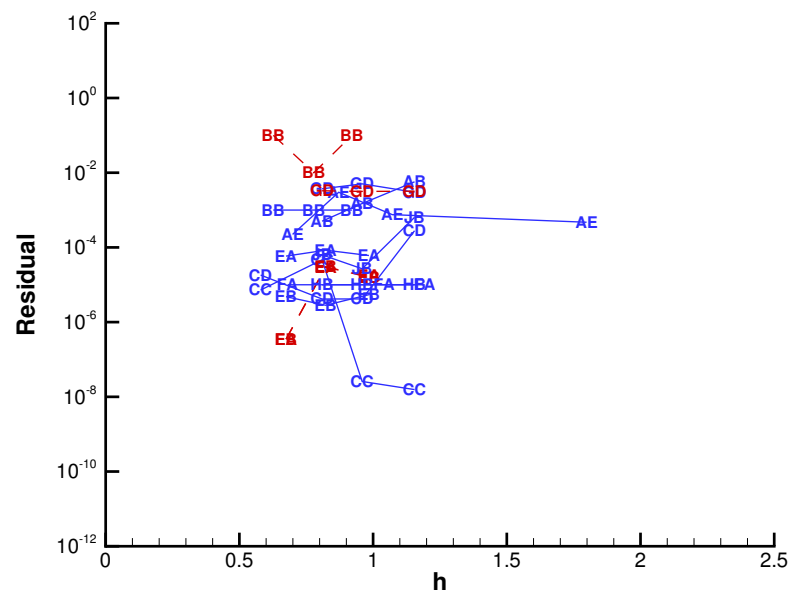

b) Residual.

Figure 21. JWB lift and iterative convergence, -Euler, -RANS. 


\section{C25F Submissions}

The submissions for the C25F model are listed in Table 8. Inviscid and viscous grid families with WS-Mixed and WS-Tet topology are provided for this case as detailed in the Required Grids section. This complex, required model has the most submissions.

Table 8. C25F submissions.

\begin{tabular}{|c|c|c|c|c|}
\hline Case & Code & Physics & Grid & Millions of Control Volumes $(h)$ \\
\hline $\mathrm{AC}$ & DLR-TAU-BJ & Euler & WS-Mixed & $103.7(0.46), 51.5(0.58), 26.9(0.72), 13.1(0.91), 6.3(1.17), 3.4(1.43)$ \\
\hline $\mathrm{AD}$ & DLR-TAU-BJ & Euler & WS-Tet & $26.9(0.72), 13.1(0.91), 6.3(1.17), 3.4(1.43)$ \\
\hline $\mathrm{AE}$ & DLR-TAU-V & RANS-SA & WS-Mixed & $69.8(0.52), 36.5(0.65), 17.9(0.82), 4.8(1.28)$ \\
\hline $\mathrm{AF}$ & DLR-TAU-V & RANS-SA & WS-Tet & $36.5(0.65), 17.9(0.82), 4.8(1.28)$ \\
\hline BB & HUNS3D & RANS-SA & HUNS3D-Mixed & $40.6(0.63), 30.4(0.69), 22.3(0.76)$ \\
\hline $\mathrm{CA}$ & FUN3D-VA & Euler & WS-Mixed & $103.7(0.46), 51.5(0.58), 26.9(0.72), 13.1(0.91), 6.3(1.17), 3.4(1.43)$ \\
\hline CB & FUN3D-VL & Euler & WS-Mixed & 103.7(0.46), 51.5(0.58), 26.9(0.72), 13.1(0.91), 6.3(1.17), 3.4(1.43) \\
\hline $\mathrm{CC}$ & FUN3D-VA & Euler & WS-Tet & 103.7(0.46), 51.5(0.58), 26.9(0.72), 13.1(0.91), 6.3(1.17), 3.4(1.43) \\
\hline $\mathrm{CE}$ & FUN3D-VA & RANS-SA & WS-Mixed & 69.8(0.52), 36.5(0.65), 17.9(0.82), 8.9(1.04), 4.8(1.28) \\
\hline $\mathrm{CF}$ & FUN3D-VL & RANS-SA & WS-Mixed & 69.8(0.52), 36.5(0.65), 17.9(0.82), 8.9(1.04), 4.8(1.28) \\
\hline CG & FUN3D-VA & RANS-SA & WS-Tet & 69.8(0.52), 36.5(0.65), 17.9(0.82), 8.9(1.04), 4.8(1.28) \\
\hline $\mathrm{CH}$ & FUN3D-VA & RANS-SA & ANSA-Mixed & $36.6(0.65)$ \\
\hline CI & FUN3D-VA & RANS-SA & Cliff-PW-Tet & $20.9(0.78)$ \\
\hline DA & JTAS & Euler & WS-Mixed & 103.7(0.46), 51.5(0.58), 26.9(0.72), 13.1(0.91), 6.3(1.17), 3.4(1.43) \\
\hline DB & JTAS & Euler & WS-Tet & $51.5(0.58), 26.9(0.72), 13.1(0.91), 6.3(1.17), 3.4(1.43)$ \\
\hline EA & LAVA-HWCNS & RANS-SA & CGT-Overset & $48.5(0.59), 25.4(0.73)$ \\
\hline EB & LAVA-Roe & RANS-SA & CGT-Overset & 48.5(0.59), 25.4(0.73) \\
\hline ED & LAVA & RANS-SA & STAR-CCM+Poly & $26.7(0.72), 14.2(0.89), 10.9(0.97)$ \\
\hline FA & Cart3D & Euler & Adapt-Cart & $18.9(0.81), 7.6(1.10), 3.8(1.38)$ \\
\hline GA & CartOVER & Euler & ft-Cart+Struct & $225.9(0.35), 122.0(0.43), 67.4(0.53), 38.1(0.64), 22.4(0.76), 12.8(0.92)$ \\
\hline GB & CartOVER & Euler & pre-Cart+Struct & 195.3(0.37), 131.2(0.42), 97.3(0.47), 78.8(0.50), 68.7(0.53), 62.4(0.54) \\
\hline $\mathrm{GC}$ & CartOVER & Euler & Adj-Cart+Struct & $225.9(0.35), 91.5(0.48), 65.9(0.53)$ \\
\hline $\mathrm{HC}$ & CFD++ & RANS-RKE & WS-Mixed & $36.5(0.65), 17.9(0.82), 8.9(1.04), 4.8(1.28)$ \\
\hline IA & USM3D & Euler & WS-Tet & 159.1(0.40), 37.1(0.65), 20.0(0.79) \\
\hline IB & USM3D & RANS-SA & WS-Tet & $28.1(0.71)$ \\
\hline JA & FUN3D-VL-HLLC & Euler & WS-Mixed & 103.7(0.46), 51.5(0.58), 26.9(0.72) \\
\hline $\mathrm{JC}$ & Cart3D & Euler & Adapt-Cart & $80.2(0.50)$ \\
\hline JD & Cart3D & Euler & Fixed-Cart & $20.0(0.79)$ \\
\hline KA & Wolf & Euler & WS-Tet & $26.9(0.72), 13.1(0.91), 6.3(1.17), 3.4(1.43)$ \\
\hline KB & Wolf-V6 & Euler & WS-Tet & 13.1(0.91), 6.3(1.17), 3.4(1.43) \\
\hline $\mathrm{KC}$ & Wolf & RANS-SA & WS-Tet & $8.9(1.04), 4.8(1.28)$ \\
\hline $\mathrm{KD}$ & Wolf-V6 & RANS-SA & WS-Tet & $8.9(1.04), 4.8(1.28)$ \\
\hline
\end{tabular}

The nearfield ensemble mean and standard deviation are shown in Fig. 22 near the centerline, Fig. 23 at intermediate offtrack angles, and Fig. 24 at large offtrack angles. This model has similar sample sizes for each physical model: 17 Euler and 14 RANS fine grid submissions. The differences in the Euler and RANS means is largest near the centerline and persists at all extraction distances. The difference between the means of the two physical modeling methods is larger than the standard deviation of either group. The differences are largest in the range of TAU=[0.8-1.0].

Figures 25 and 26 show selected signatures at $\mathrm{PHI}=0^{\circ}$ and $\mathrm{PHI}=50^{\circ}$, respectively, extracted from inviscid simulations at $\mathrm{R}=5$. One signature per participant group is shown. For participants who submitted multiple cases, we show the case with the least discretization error and we also try to balance signatures from WS-Mixed and WS-Tet grids. In cases where the discretization error analysis diverged and the participants submitted results on more than three meshes, we shifted the analysis to include the next coarser grid. The number of control volumes in the grids used to plot each signature is noted in the captions of Figs. 25 and 26.

In general, there is good agreement in the signatures among the participant groups at both $\mathrm{PHI}=0^{\circ}$ and $\mathrm{PHI}=50^{\circ}$. On-track signatures show the greatest sensitivity to grid refinement in the range of TAU=[0.8-1.0], as shown in Fig. 25 and also previously in Fig. 22. Furthermore, there are also areas of notable discretization error along the linear pressure ramp upstream of TAU $=0.4$ (see Figs. 25a, 25c, 25g, and 25h), indicating sensitivity to grid refinement and the surface tessellation, and in the wake (downstream of TAU=1.2). For case JA in Fig. 25g, the level of error is increasing as the grid is refined and consequently the error estimate is marked "Low Confidence." Figure 26 shows that the agreement among the signatures improves significantly at $\mathrm{PHI}=50^{\circ}$, with most discretization error being flagged in the wake.

Figure 27 shows selected signatures extracted from RANS simulations at $\mathrm{R}=5$ and $\mathrm{PHI}=0^{\circ}$. The degree of grid convergence of signatures from RANS simulations is not as good as those from Euler simulations. The level of error as the grid is refined increases in approximately $50 \%$ of the signatures. Cases AE, CE and CF use WS-Mixed grids, while case ED uses a STAR-CCM+Poly grid - these all generate very similar signatures, and we show case CE as 
an example in Fig. 27c. Cases AF and CG use the WS-Tet grids, and these also generate very similar signatures. Figure 27a shows the signature from case AF, which is in good agreement with Fig. 27c. Cases EA and EB predict signatures with a different shock pattern in the region TAU $=[0.8-1.0]$ and deeper expansions in the region TAU $=[1.0-$ 1.2]. Furthermore, case EA contains a large overshoot near TAU $=1.1$. The signature from Case HC in Fig. $27 \mathrm{f}$ agrees fairly well with those in Figs. 27a and 27c, but the discretization error analysis determines an unusually small observed order of accuracy of 0.21. Consequently, the error estimate is marked "Low Confidence" in Fig. 27f.

The PL grid convergence of the submissions is shown in Fig. 28 near the centerline, Fig. 29 at intermediate offtrack angles, and Fig. 30 at large offtrack angles. The Euler submissions are shown in blue and the RANS submissions are shown in red. The RANS submissions tended to have a higher PL than Euler at PHI of $0^{\circ}, 10^{\circ}$, and $50^{\circ}$. The Euler and RANS appear to convergence to similar values for PHI of $20^{\circ}, 30^{\circ}$, and $40^{\circ}$. The $0^{\circ}$ and $10^{\circ} \mathrm{PHI}$ Euler PL appear to curve upward at an $h$ of 0.5 indicating that a new flow feature may be resolved on these finer grids. This observation appears to be supported by the relatively large discretization error estimates in the range TAU $=[0.8-1.1]$ in Fig. 25. The linear error transport equation method of Derlaga, Park, and Rallabhandi [60] implicated the underresolved inlet shock that reflected off the wing and horizontal tail as a strong driver of the Euler C25F variation due to grid refinement. New points of inflection appeared in fine grid propagated ground signatures that were not seen on coarser grids. The EA and EB overset grid PL cases are louder than the other RANS cases, especially at smaller offtrack angle. This can be attributed to the differences in their nearfield signatures shown in Fig. 27.

Box, whisker, and violin plots of PL calculated from the fine grid Euler and RANS submissions are shown in Fig. 31. The PL values are plotted for each offtrack angle to produce PL carpet plots with submissions provided at one, three, and five body lengths. The interquartile range (box extent) is larger for the Euler submissions at $0^{\circ}-$ $20^{\circ} \mathrm{PHI}$ than $30^{\circ}-50^{\circ} \mathrm{PHI}$. There are more outliers for the RANS physical model than the Euler physical model. The high RANS outliers are EA and EB cases for most PHI and R and case BB is low for most PHI and R.

The convergence of lift and relative iterative convergence for the $\mathrm{C} 25 \mathrm{~F}$ submissions is shown in Fig. 32. The Euler submissions trend toward a higher lift value than the RANS submissions with the exception of JC and JD. The relative meanflow equation iterative convergence is shown in blue solid lines and the turbulence model relative convergence is shown in red with dashed lines. 


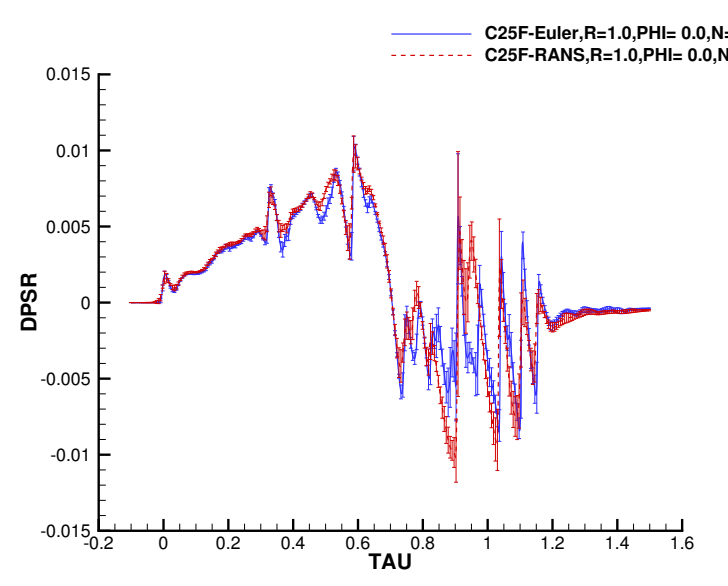

a) $\mathrm{R}=1, \mathrm{PHI}=\mathbf{0}^{\circ}$.

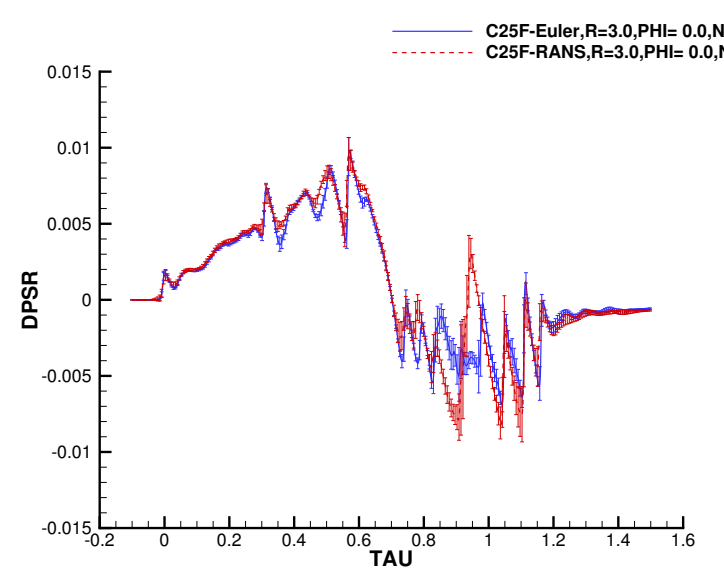

c) $\mathbf{R}=3, \mathbf{P H I}=0^{\circ}$.

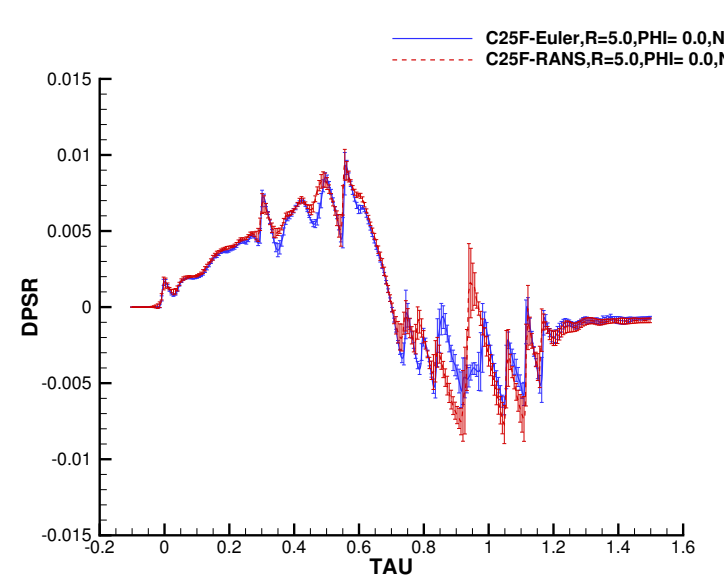

e) $\mathrm{R}=5, \mathrm{PHI}=0^{\circ}$.

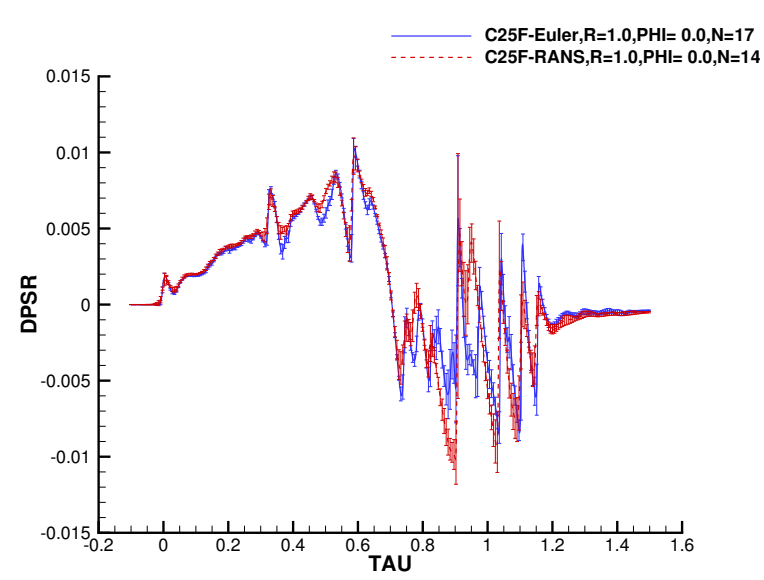

b) $\mathrm{R}=1, \mathrm{PHI}=10^{\circ}$.

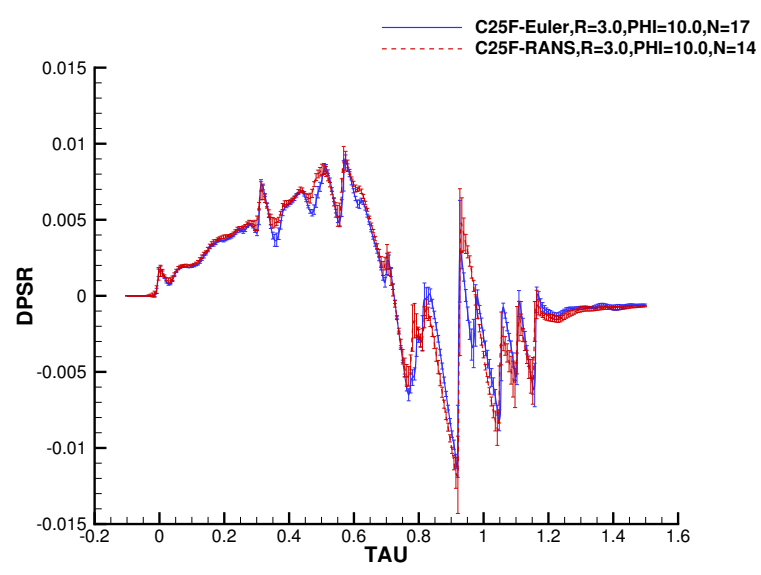

d) $\mathrm{R}=3, \mathrm{PHI}=10^{\circ}$.

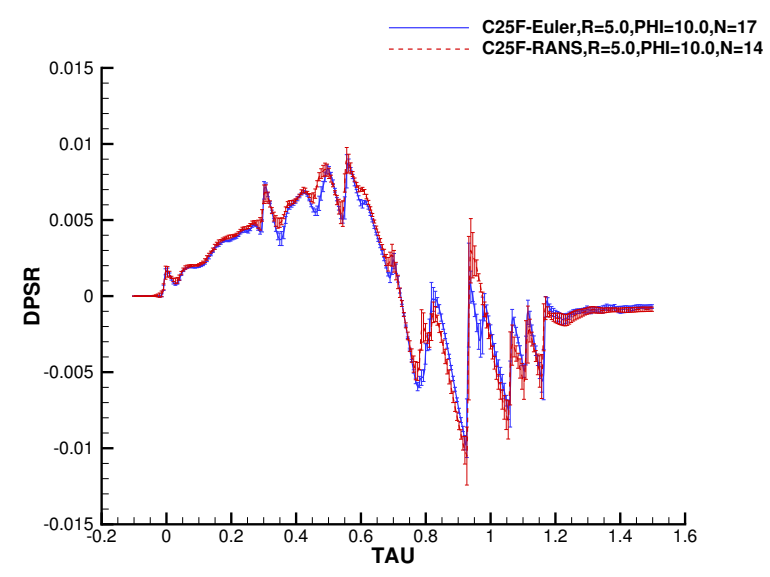

f) $\mathrm{R}=5, \mathrm{PHI}=10^{\circ}$.

Figure 22. $\mathrm{C} 25 \mathrm{~F}$ nearfield ensemble mean and standard deviation for fine grids, $\mathrm{PHI}=0^{\circ}-10^{\circ}$. 


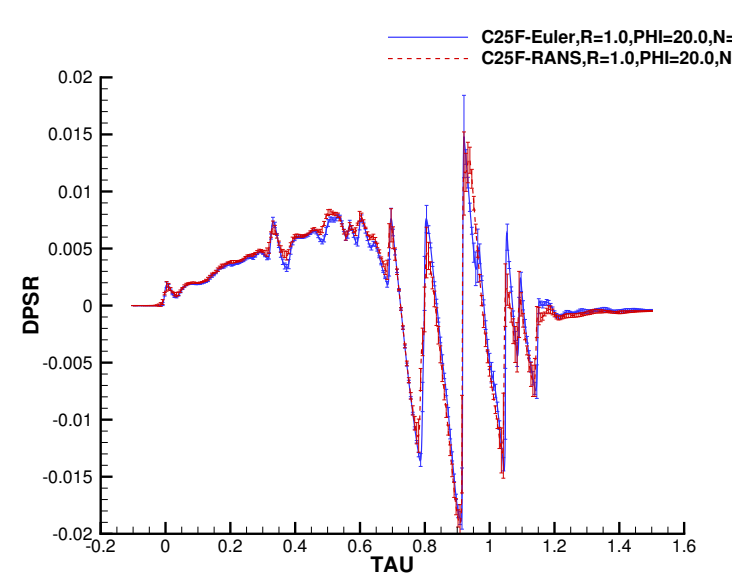

a) $\mathbf{R = 1}, \mathbf{P H I}=\mathbf{2 0}{ }^{\circ}$.

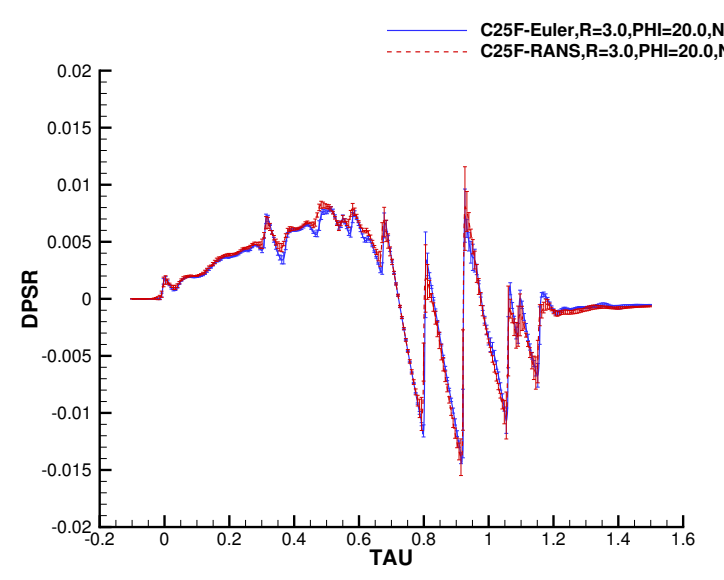

c) $\mathrm{R}=3, \mathbf{P H I}=20^{\circ}$.

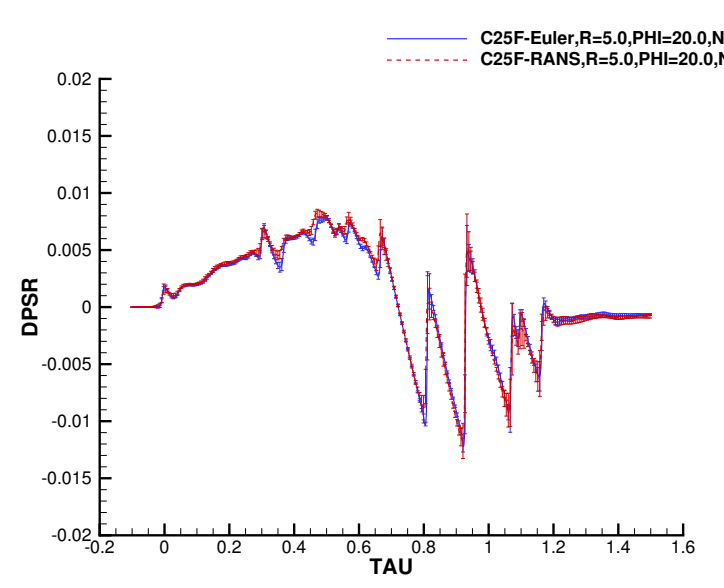

e) $\mathbf{R}=5$, $\mathrm{PHI}=20^{\circ}$.

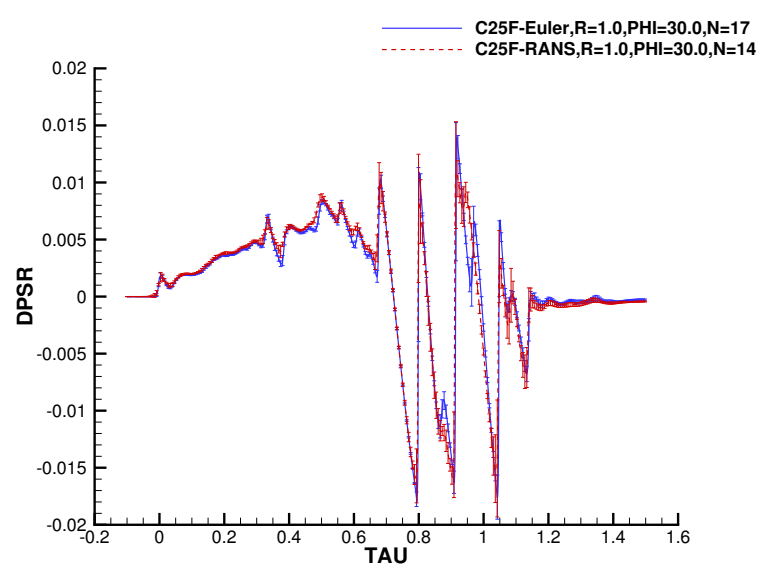

b) $\mathrm{R}=1, \mathrm{PHI}=30^{\circ}$.

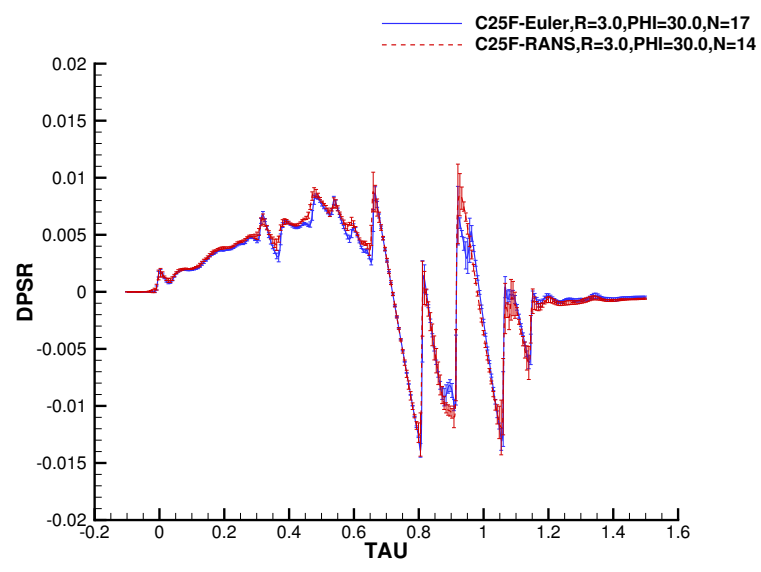

d) $\mathrm{R}=3, \mathrm{PHI}=30^{\circ}$.

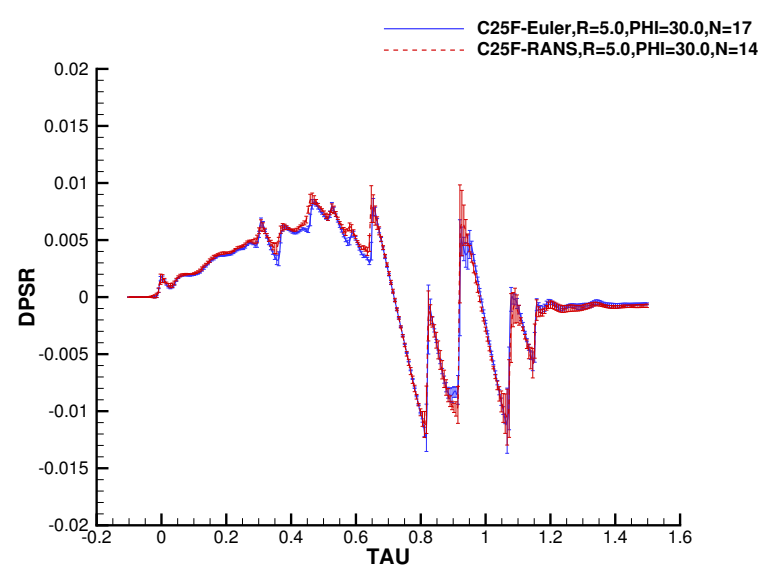

f) $\mathbf{R}=5, \mathrm{PHI}=30^{\circ}$.

Figure 23. $\mathrm{C25F}$ nearfield ensemble mean and standard deviation for fine grids, $\mathrm{PHI}=20^{\circ}-30^{\circ}$. 


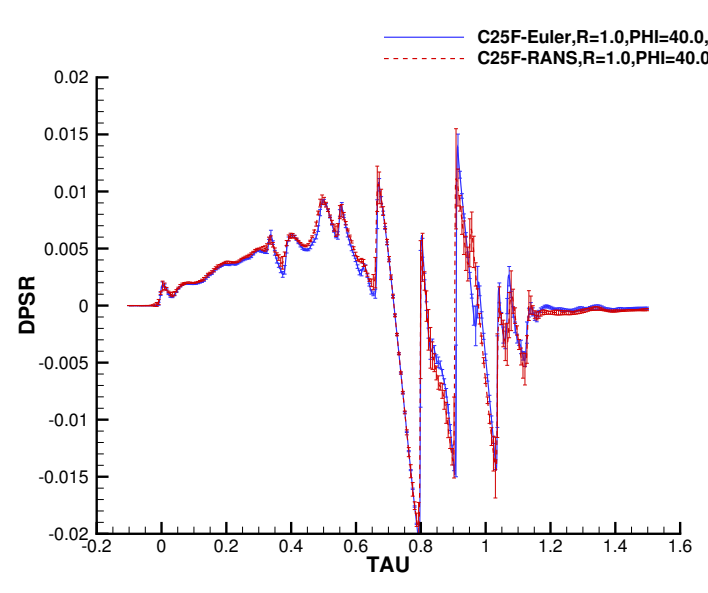

a) $\mathrm{R}=\mathbf{1}, \mathbf{P H I}=\mathbf{4 0}^{\circ}$.

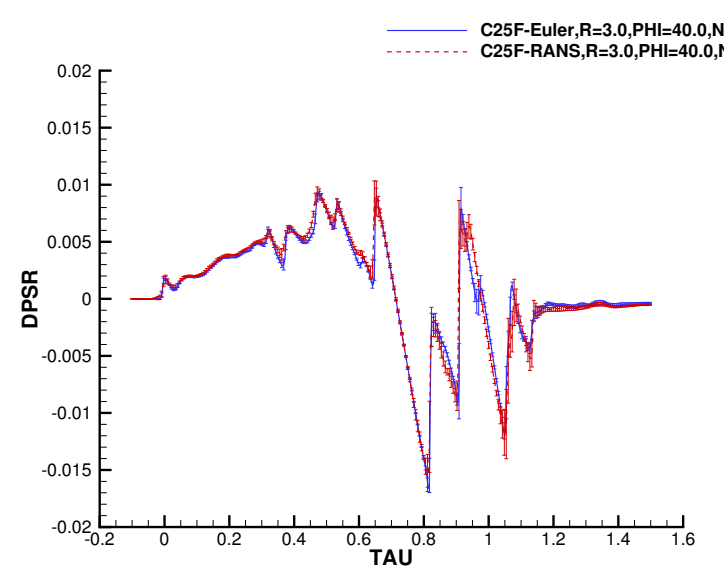

c) $\mathrm{R}=3, \mathrm{PHI}=\mathbf{4 0}{ }^{\circ}$.

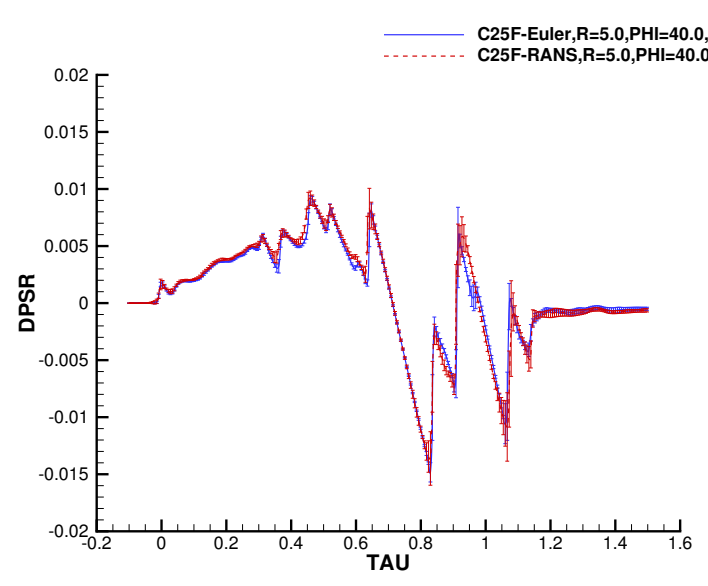

e) $\mathbf{R}=5$, $\mathrm{PHI}=\mathbf{4 0}$.

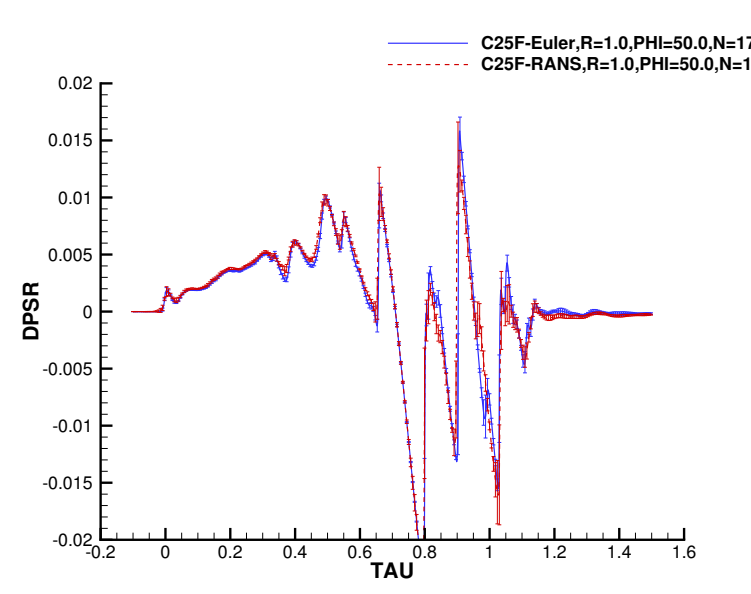

b) $\mathrm{R}=1, \mathrm{PHI}=50^{\circ}$.

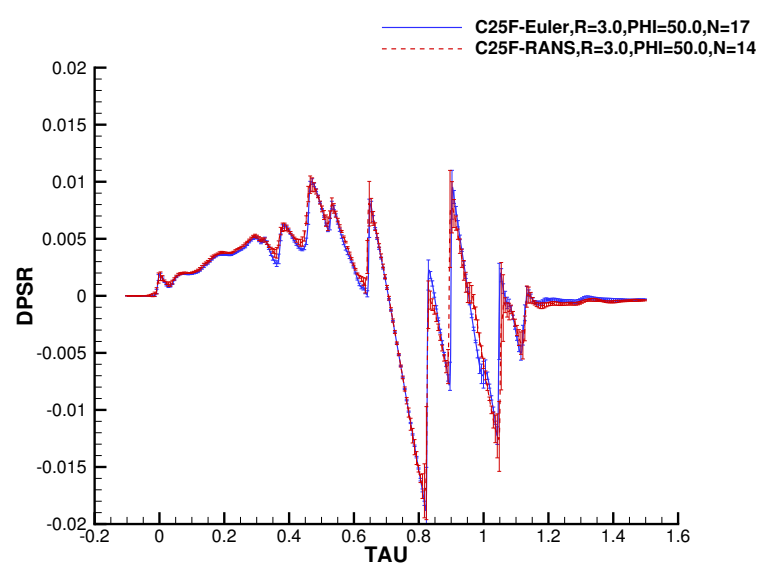

d) $\mathrm{R}=3, \mathrm{PHI}=50^{\circ}$.

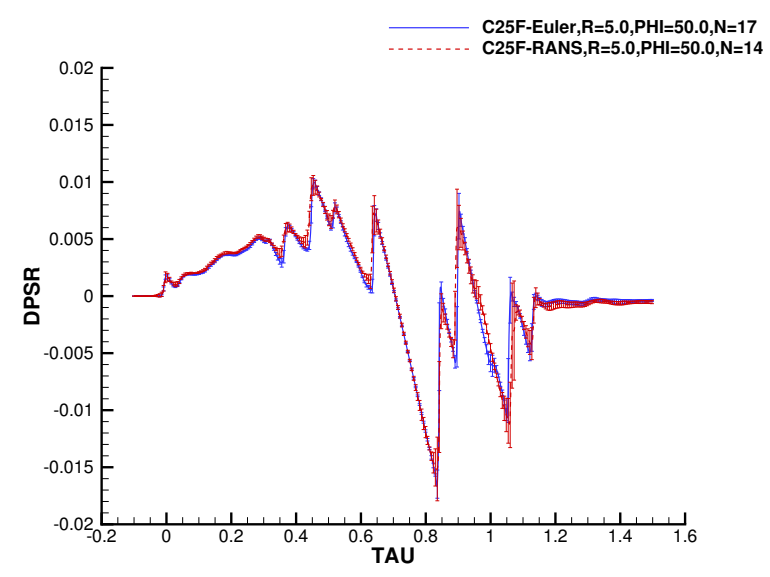

f) $\mathbf{R}=5, \mathrm{PHI}=50^{\circ}$.

Figure 24. $\mathrm{C25F}$ nearfield ensemble mean and standard deviation for fine grids, $\mathrm{PHI}=40^{\circ}-50^{\circ}$. 


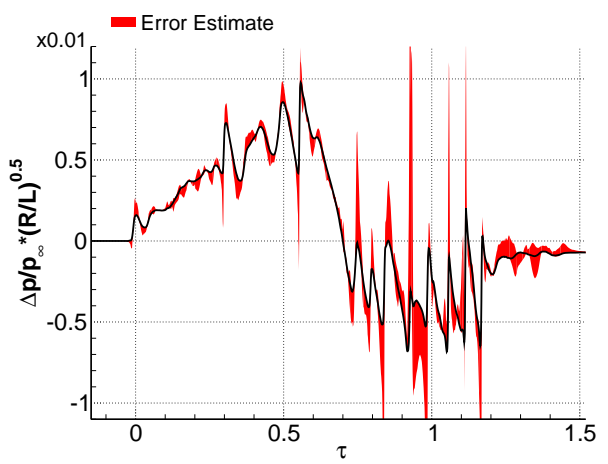

a) $\mathrm{AC}(\mathbf{5 1 . 5 M})$.

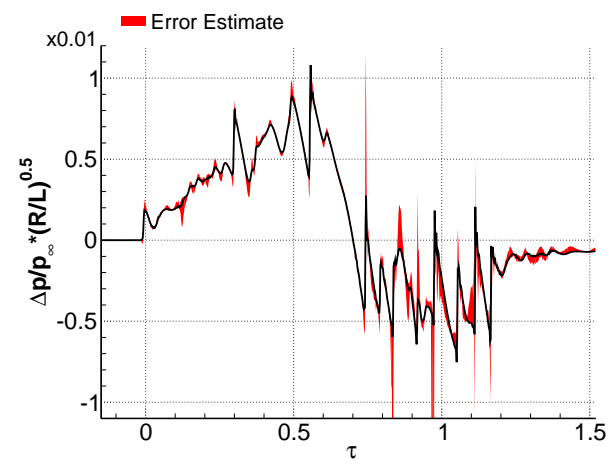

c) DA (51.5M).

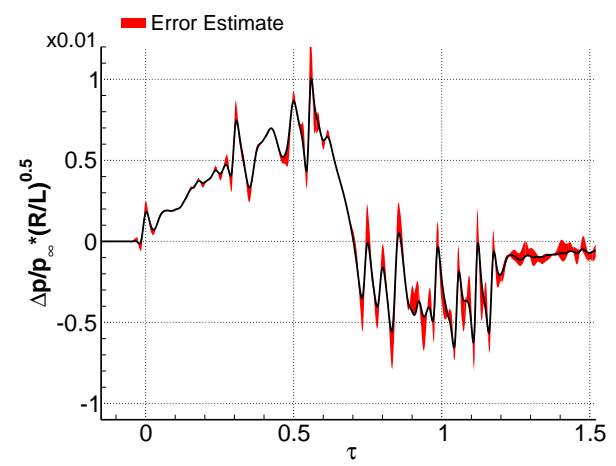

e) GA (122M).

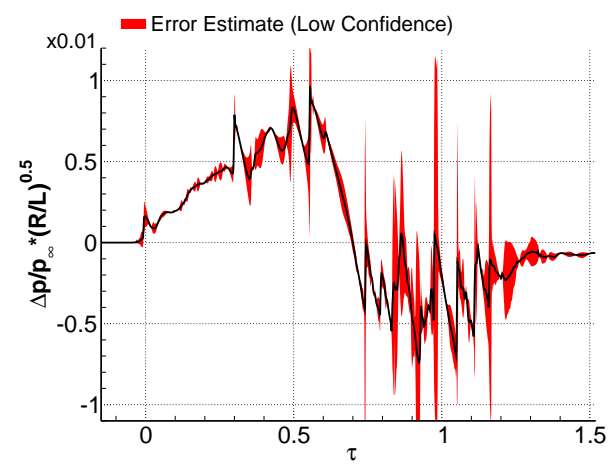

g) JA (103.9M).

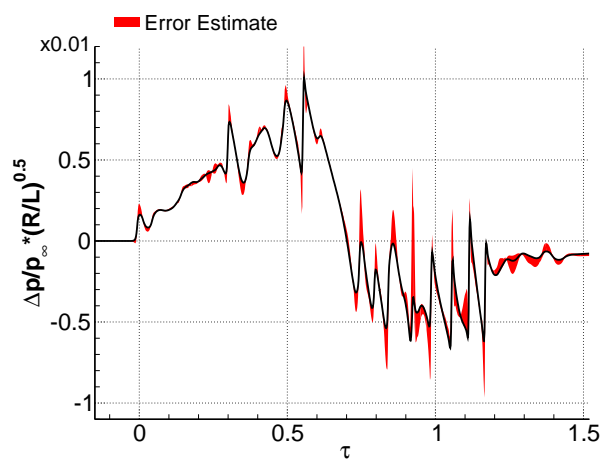

b) $\mathrm{CC}(103.7 \mathrm{M})$.

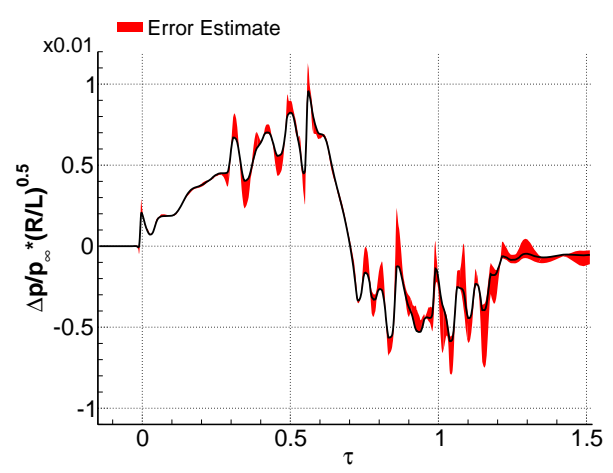

d) FA (18.9M).

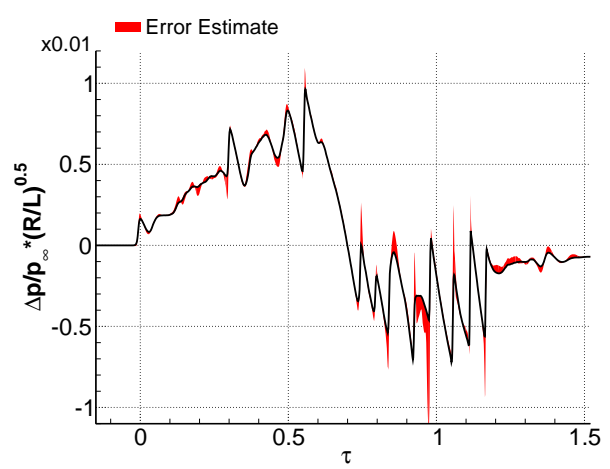

f) IA (159M).

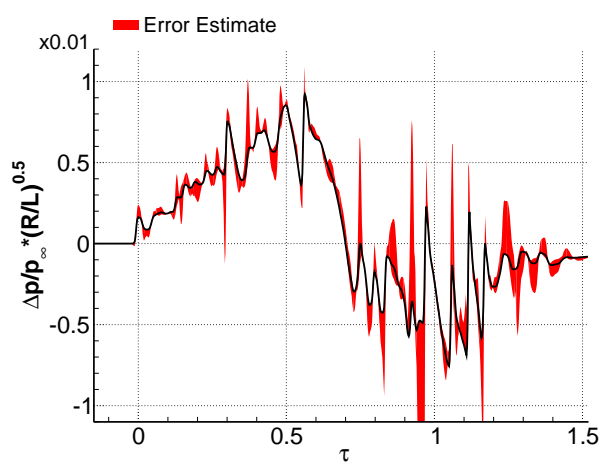

h) KB (13.1M).

Figure 25. $\mathrm{C25F}$ Euler nearfield signatures computed on fine grids with discretization error estimates: $\mathrm{R}=5, \mathrm{PHI}=\mathbf{0}^{\circ}$.

34 of 61

American Institute of Aeronautics and Astronautics 


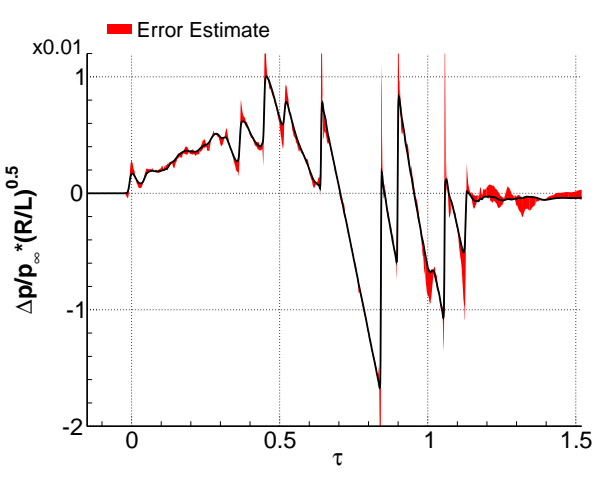

a) $\mathrm{AC}(51.5 \mathrm{M})$.

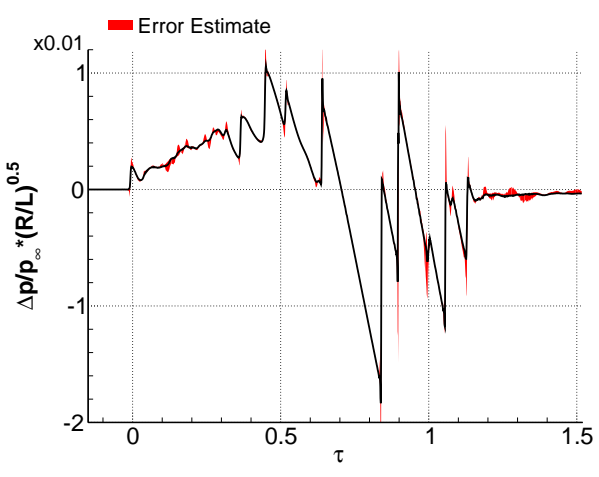

c) DA (51.5M).

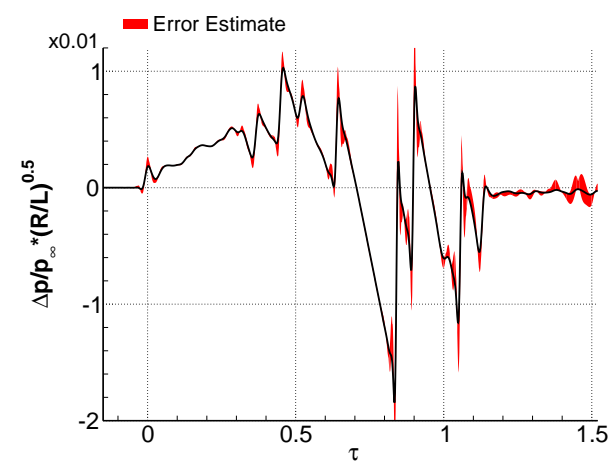

e) GA (122M).

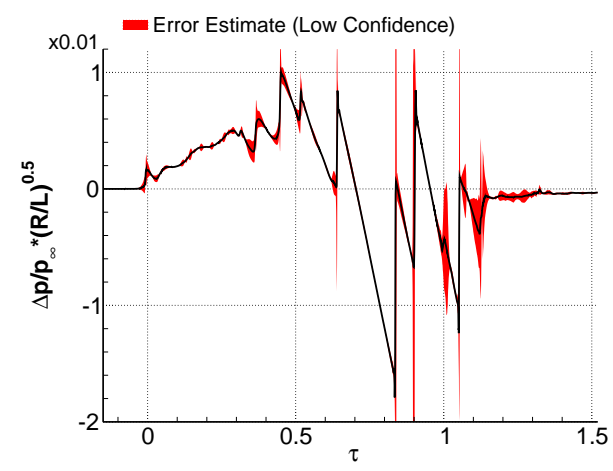

g) JA (103.9M).

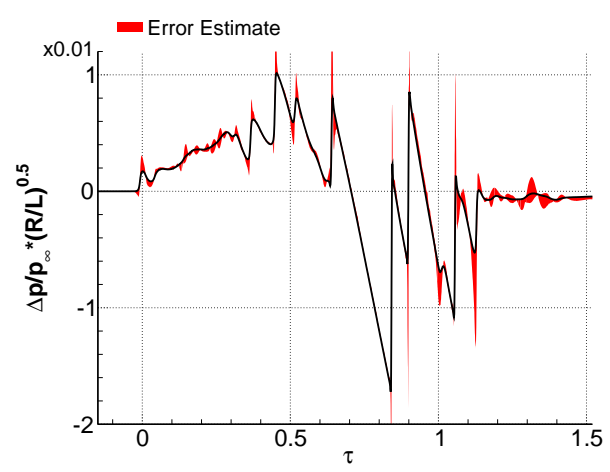

b) $\mathrm{CC}(103.7 \mathrm{M})$.

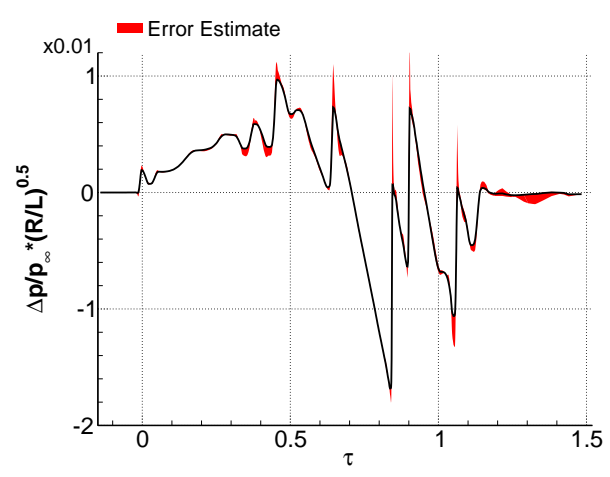

d) FA (18.9M).

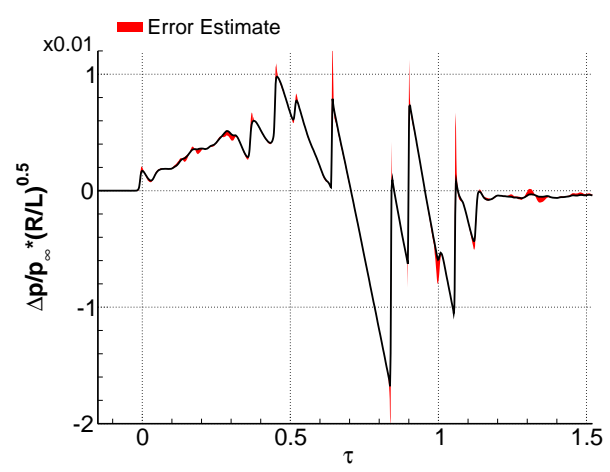

f) IA (159M).

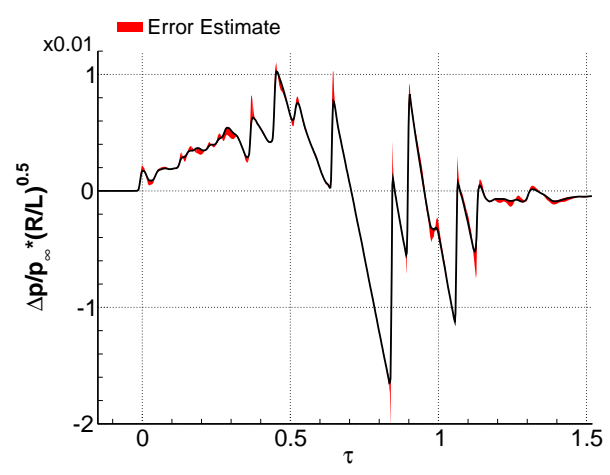

h) KB (13.1M).

Figure 26. $\mathrm{C25F}$ Euler nearfield signatures computed on fine grids with discretization error estimates: $\mathrm{R}=5, \mathrm{PHI}=\mathbf{5 0}^{\circ}$. 


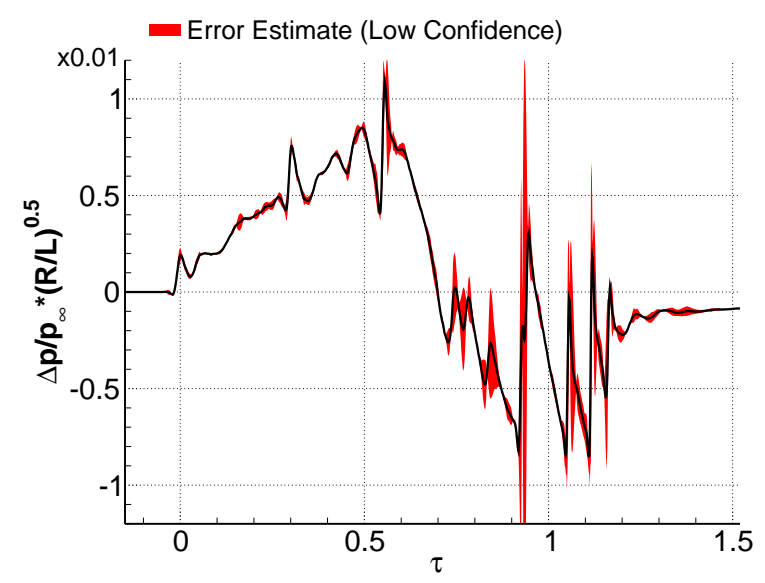

a) AF (shown, 36.5M), CG.

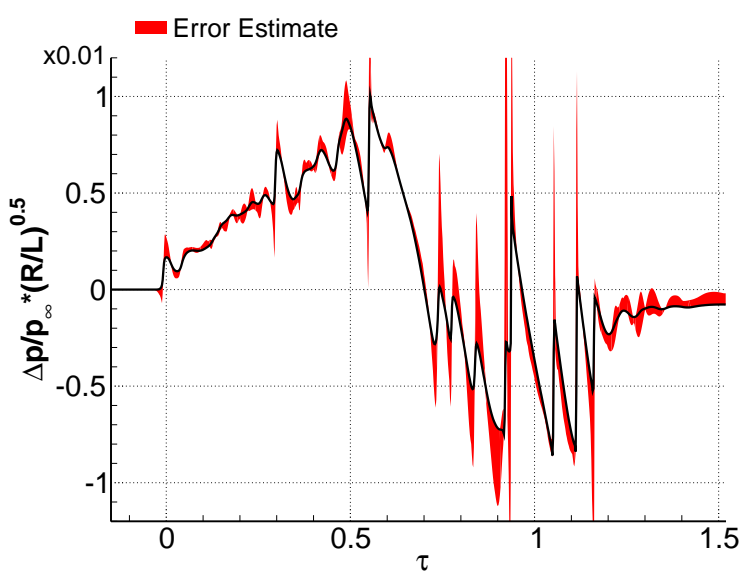

c) $\mathrm{AE}, \mathrm{CE}$ (shown, 69.8M), CF, ED.

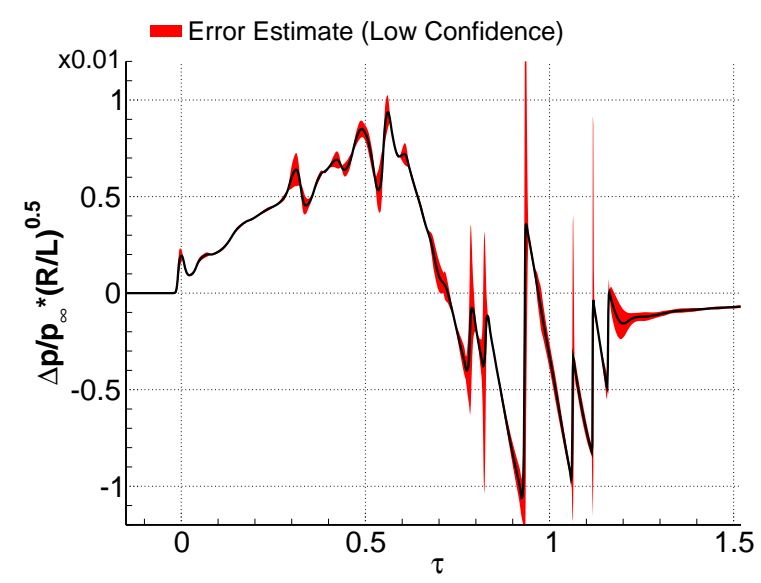

e) $\mathrm{EB}(48.5 \mathrm{M})$.

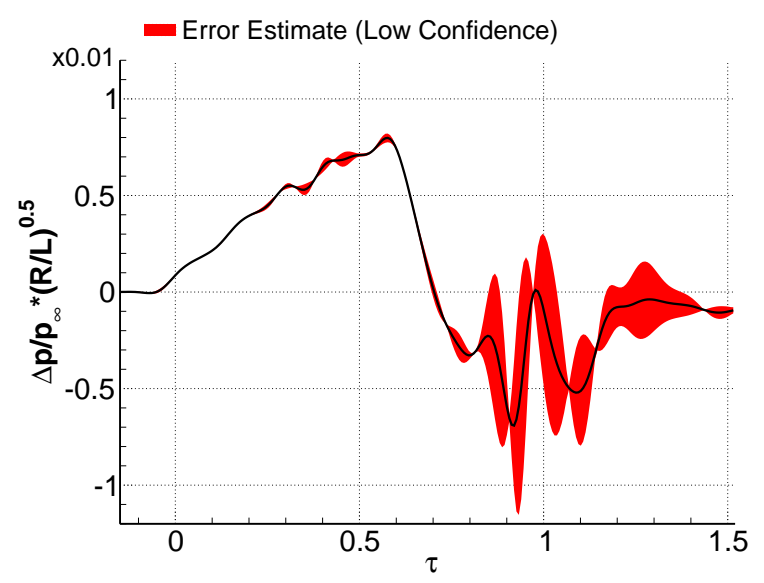

b) $\mathrm{BB}(40.6 \mathrm{M})$.

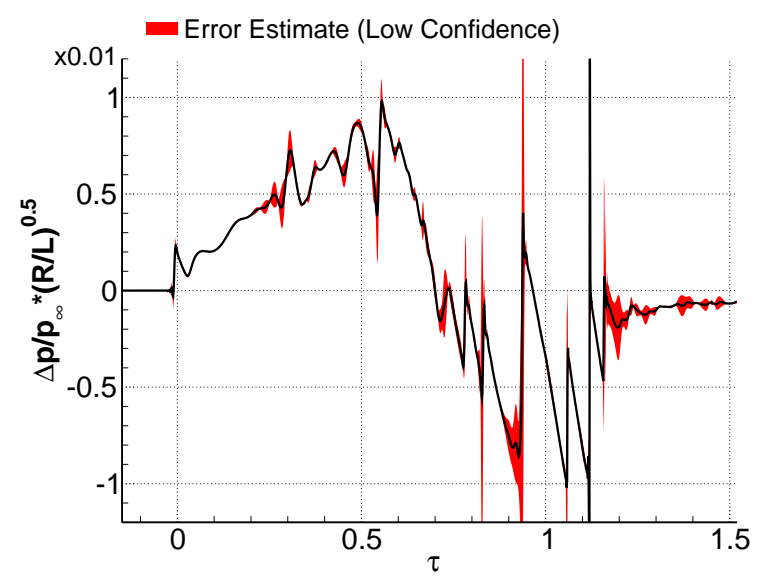

d) EA (48.5M).

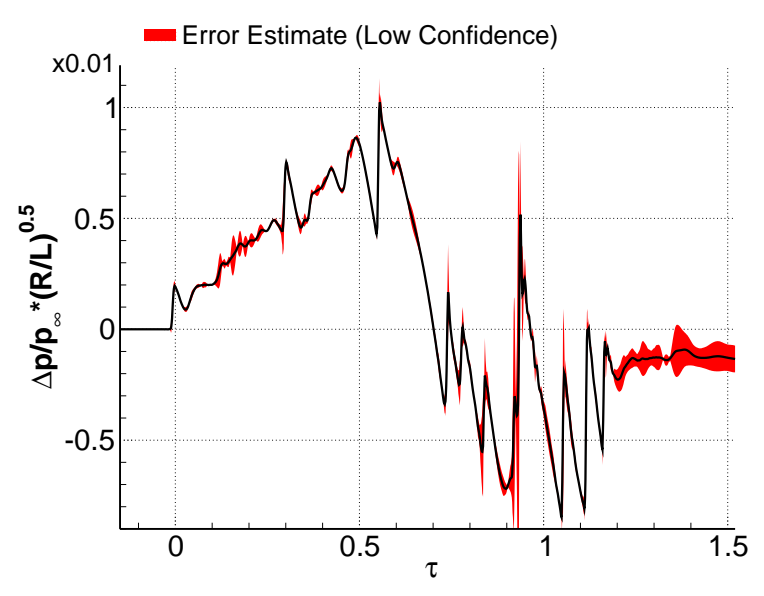

f) $\mathrm{HC}(36.5 \mathrm{M})$.

Figure 27. C25F RANS nearfield signatures computed on fine grids with discretization error estimates: $\mathrm{R}=5, \mathrm{PHI}=0^{\circ}$. 


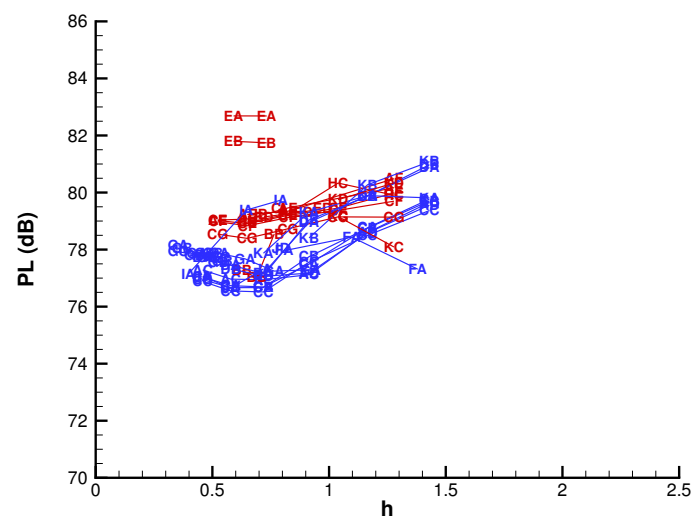

a) $\mathrm{R}=1, \mathrm{PHI}=0^{\circ}$.

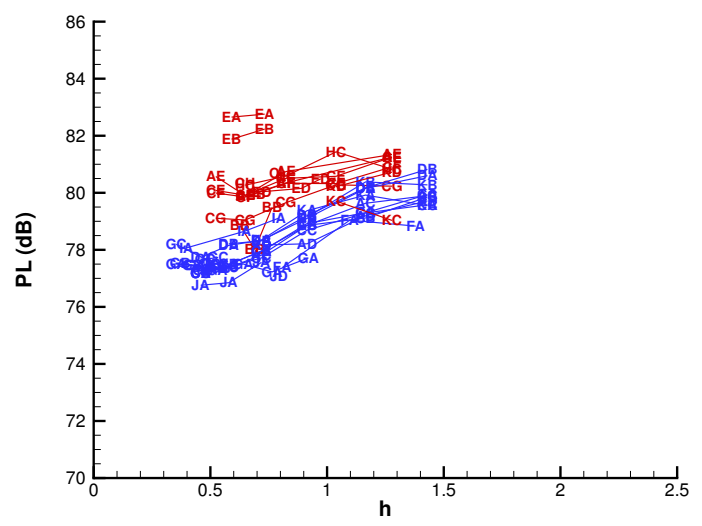

c) $\mathrm{R}=3, \mathrm{PHI}=0^{\circ}$.

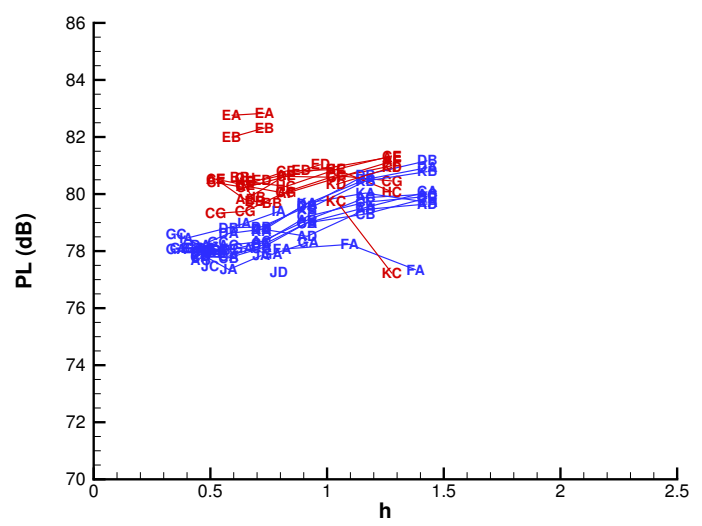

e) $\mathbf{R}=5, \mathrm{PHI}=0^{\circ}$.

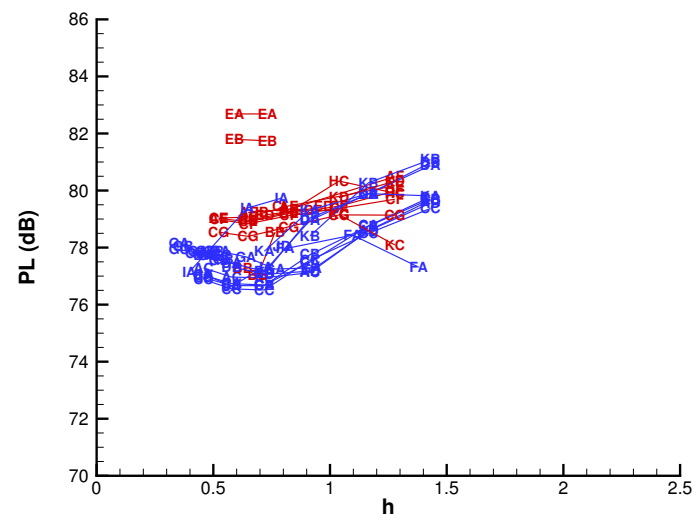

b) $\mathrm{R}=1, \mathrm{PHI}=10^{\circ}$.

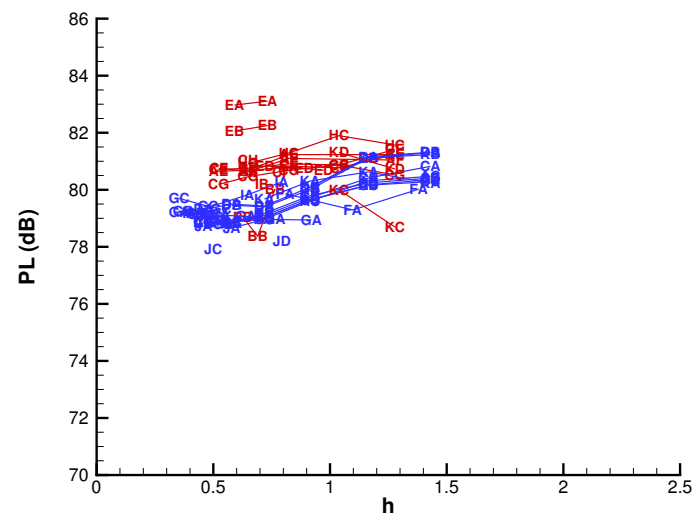

d) $\mathbf{R}=3, \mathbf{P H I}=10^{\circ}$.

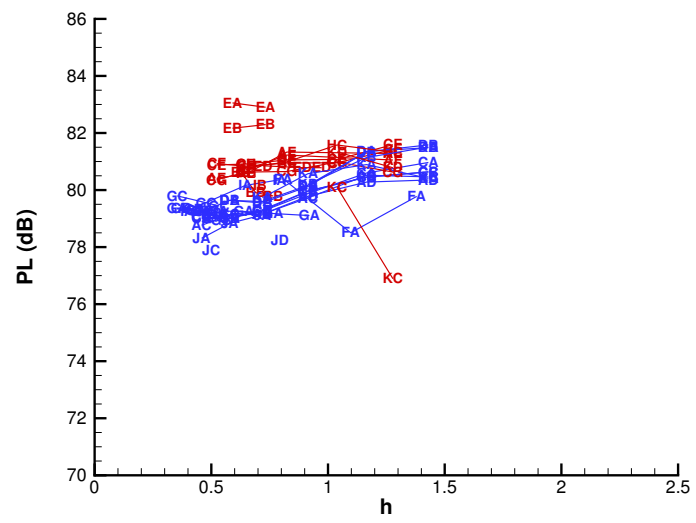

f) $\mathrm{R}=5, \mathrm{PHI}=10^{\circ}$.

Figure 28. C25F PL grid convergence, $\mathrm{PHI}=0^{\circ}-10^{\circ}$, -Euler, - RANS. 


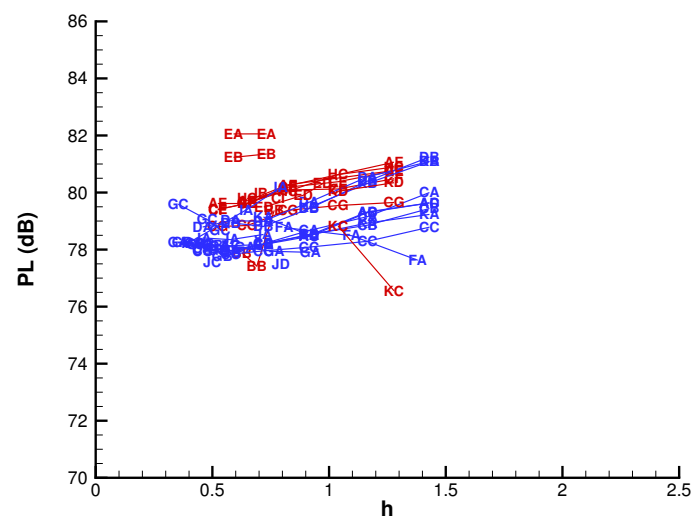

a) $\mathrm{R}=\mathbf{1}, \mathrm{PHI}=\mathbf{2 0}{ }^{\circ}$.

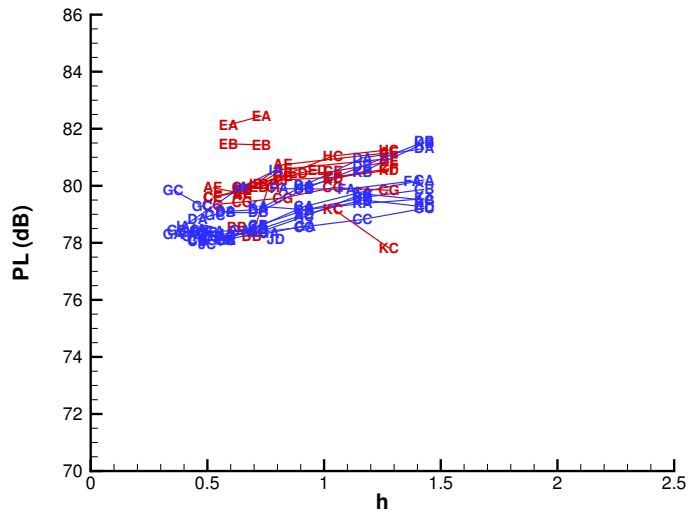

c) $\mathrm{R}=3, \mathrm{PHI}=20^{\circ}$.

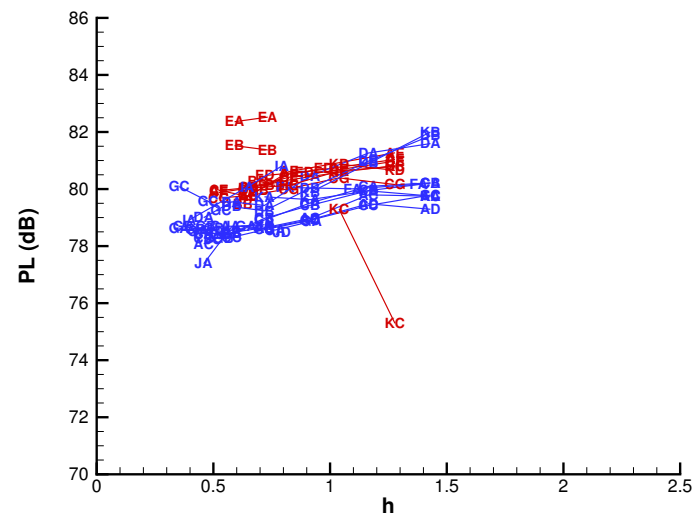

e) $\mathrm{R}=5, \mathrm{PHI}=20^{\circ}$.

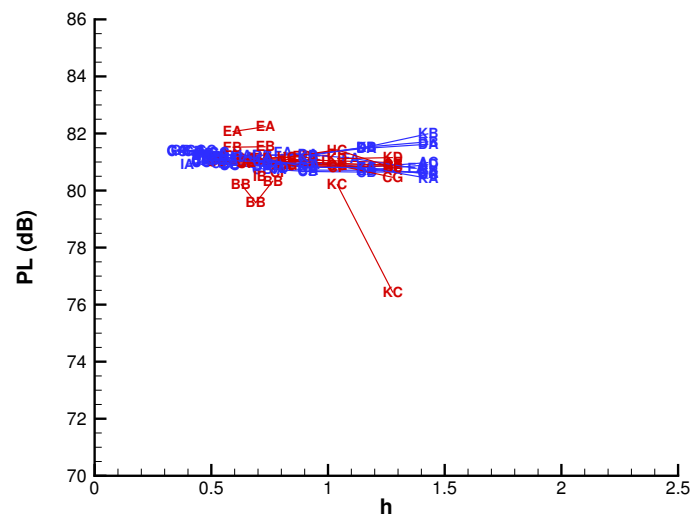

b) $\mathrm{R}=1, \mathrm{PHI}=30^{\circ}$.

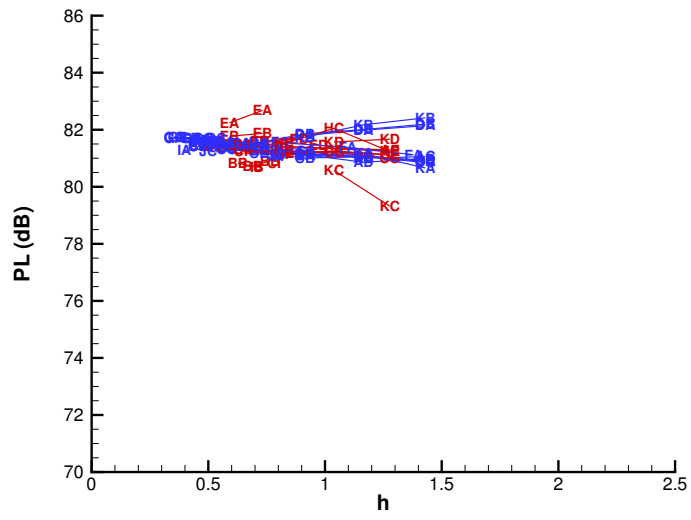

d) $\mathrm{R}=3, \mathrm{PHI}=30^{\circ}$.

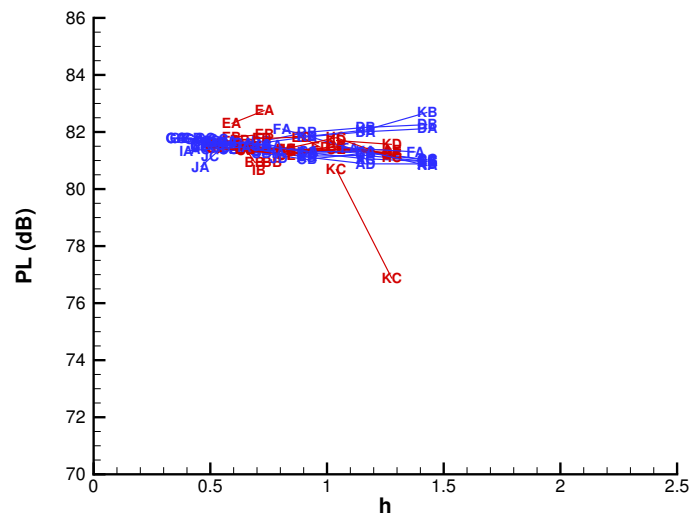

f) $\mathrm{R}=5, \mathrm{PHI}=30^{\circ}$

Figure 29. $\mathrm{C25F}$ PL grid convergence, $\mathrm{PHI}=\mathbf{2 0}^{\circ}-\mathbf{3 0}^{\circ}$, -Euler, -RANS. 


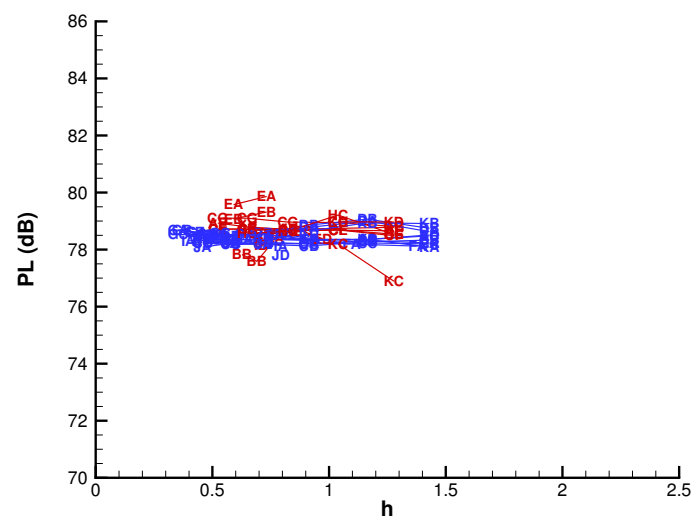

a) $\mathrm{R}=\mathbf{1}, \mathrm{PHI}=40^{\circ}$.

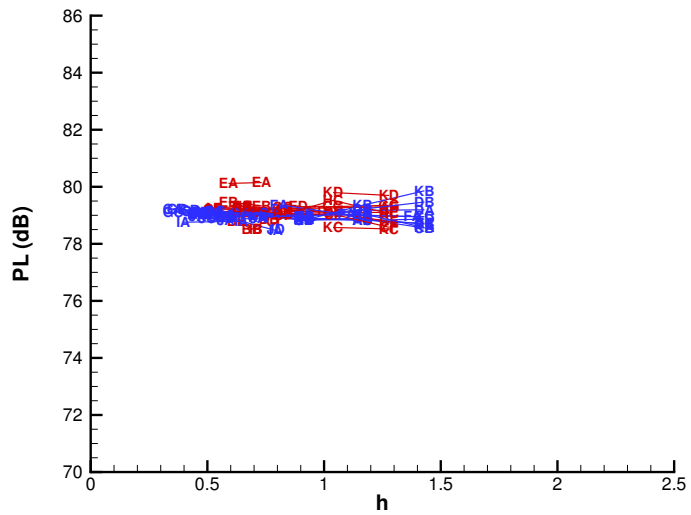

c) $\mathrm{R}=3, \mathrm{PHI}=40^{\circ}$.

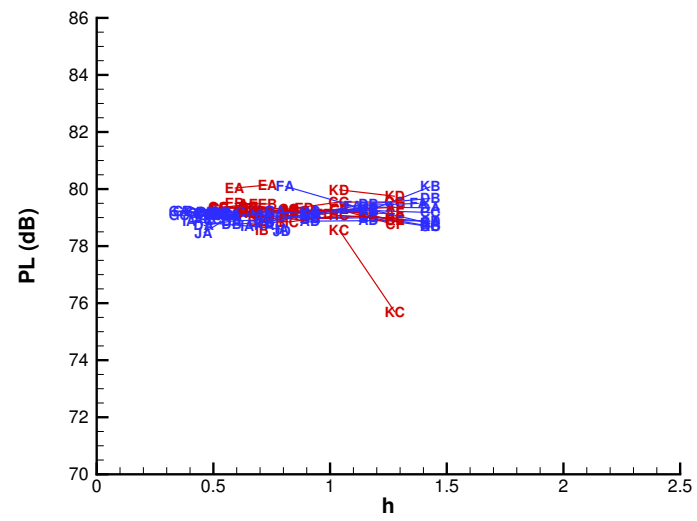

e) $\mathrm{R}=5, \mathrm{PHI}=40^{\circ}$.

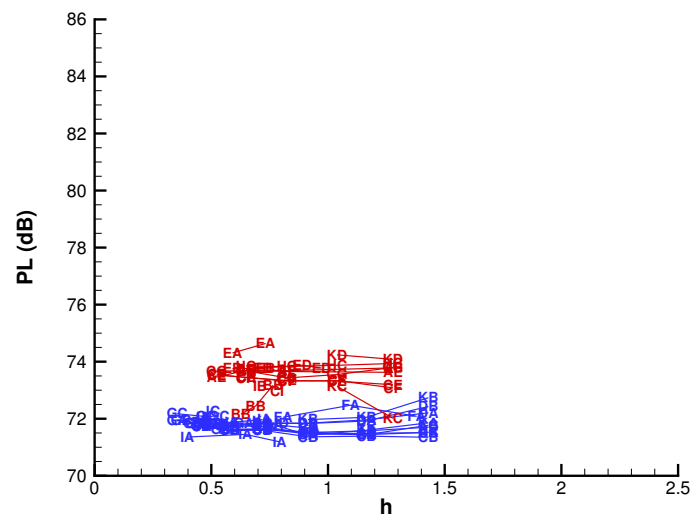

b) $\mathrm{R}=\mathbf{1}, \mathrm{PHI}=\mathbf{5 0}^{\circ}$.

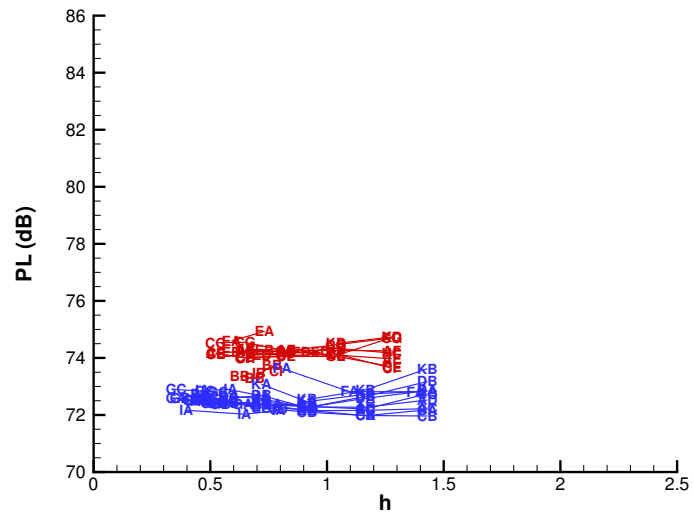

d) $\mathbf{R = 3}, \mathrm{PHI}=50^{\circ}$.

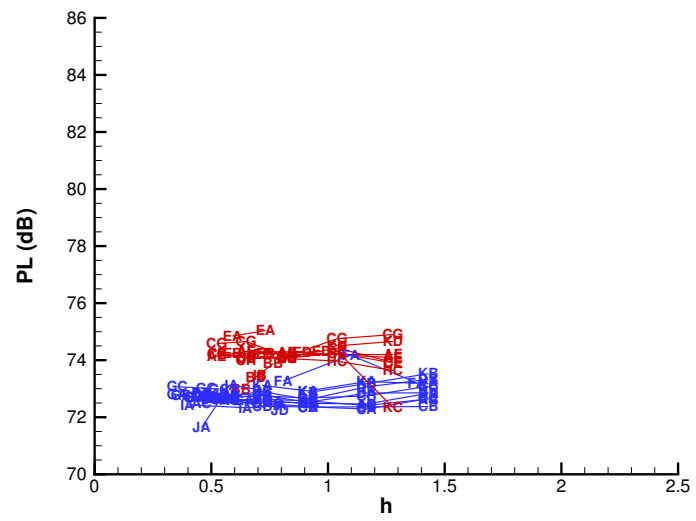

f) $\mathrm{R}=5, \mathrm{PHI}=50^{\circ}$.

Figure 30. C25F PL grid convergence, $\mathrm{PHI}=40^{\circ}-50^{\circ}$, -Euler, - RANS. 


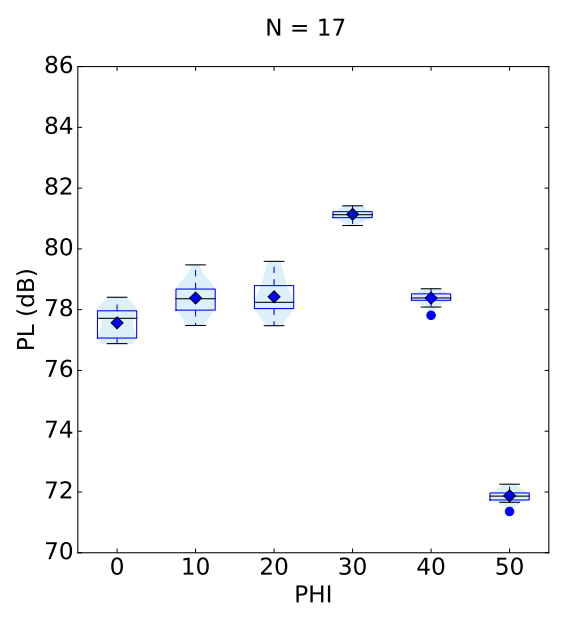

a) Euler $\mathrm{R}=1 . \mathrm{PHI}=\mathbf{4 0}^{\circ}$ : JD low. $\mathrm{PHI}=50^{\circ}$ : IA low.

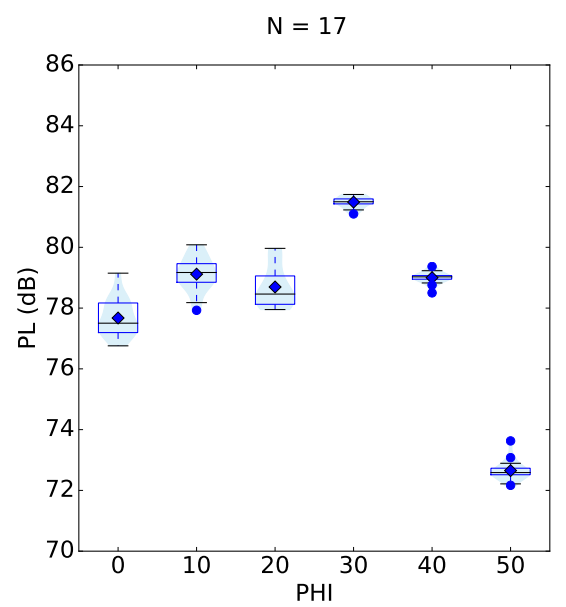

c) Euler R=3. $\mathrm{PHI}=10^{\circ}, 30^{\circ}$ : JC low. $\mathrm{PHI}=40^{\circ}$ : FA high. JD, IA low. PHI $=50^{\circ}$ : FA, KA high, IA low.

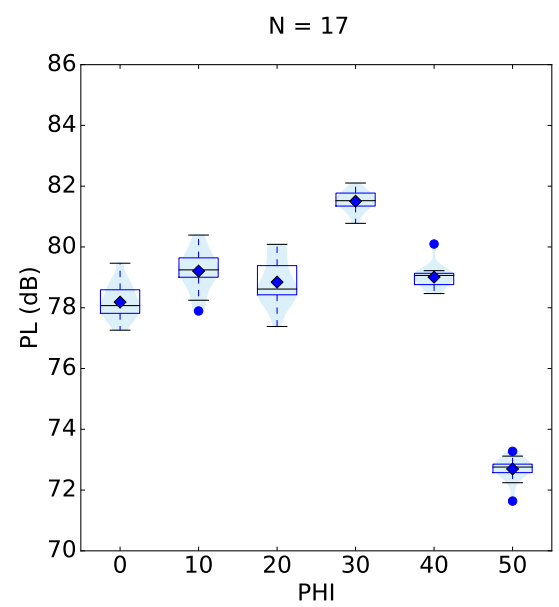

e) Euler $\mathrm{R}=5$. $\mathrm{PHI}=10^{\circ}$ : $\mathrm{JC}$ low. $\mathrm{PHI}=40^{\circ}$ : FA high. PHI $=50^{\circ}$ : FA high, JA low.

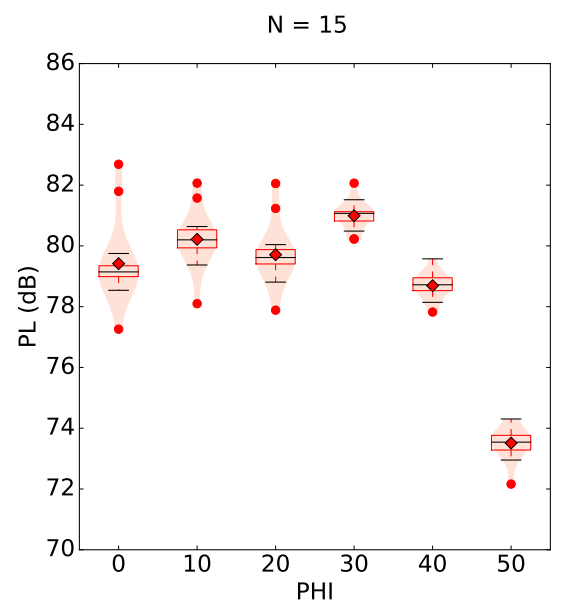

b) RANS R=1. PHI $=0^{\circ}, 10^{\circ}, 20^{\circ}$ : EA, EB high, BB low. $\mathrm{PHI}=30^{\circ}$ : $\mathrm{EA}$ high, $\mathrm{BB}$ low. $\mathrm{PHI}=4^{\circ}, \mathrm{50}^{\circ}$ : $\mathrm{BB}$ low.

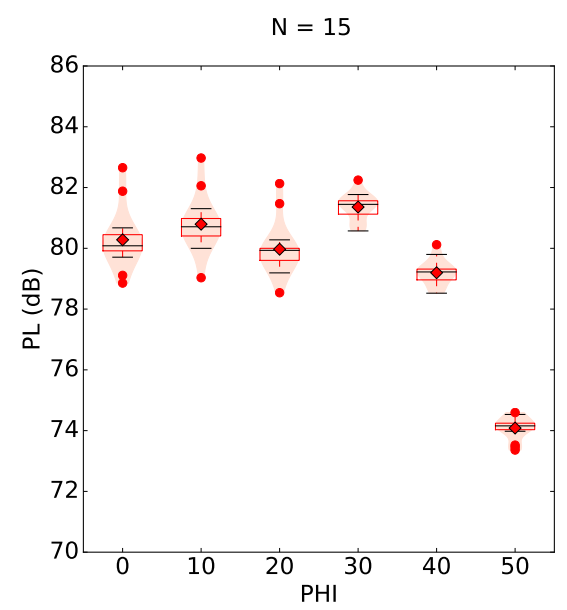

d) RANS R=3. PHI $=0^{\circ}$ : EA, EB high, BB, CG low. $\mathrm{PHI}=10^{\circ}, 2^{\circ}, 30^{\circ}$ : EA, EB high, BB low. $\mathrm{PHI}=40^{\circ}$ : EA high. $\mathrm{PHI}=50^{\circ}$ : EA high, BB, IB, CI low.

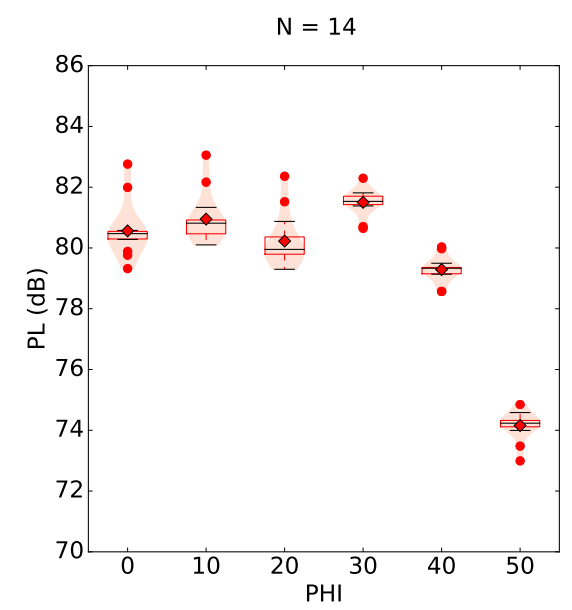

f) RANS R=5. $\mathrm{PHI}=0^{\circ}$ : EA, EB high, $\mathrm{CG}, \mathrm{KC}, \mathrm{IB}$ low. $\mathrm{PHI}=10^{\circ}, 20^{\circ}$ : EA, EB high. PHI $=30^{\circ}$ : EA high, IB, KC low. $\mathrm{PHI}=40^{\circ}$ : EA, KD high, KC low. $\mathrm{PHI}=50^{\circ}$ : EA high, BB, IB low.

Figure 31. C25F PL fine grid statistics. 


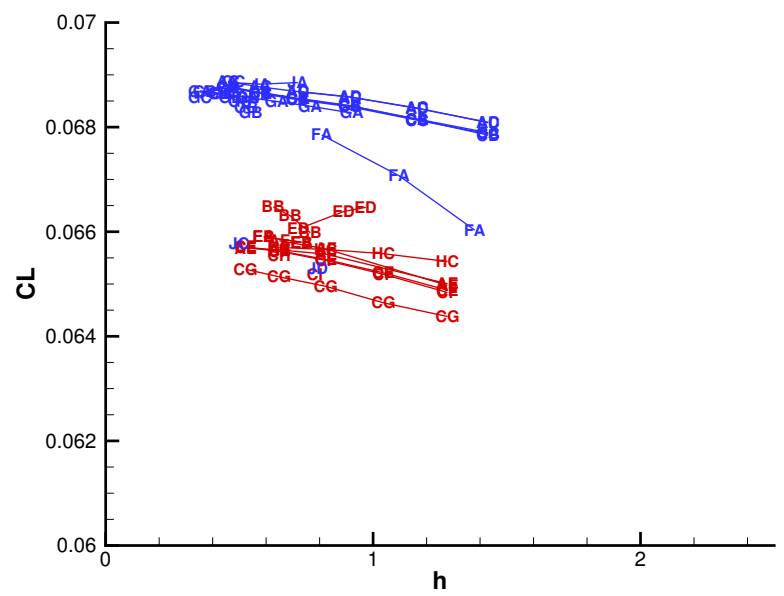

a) Lift Coefficient.

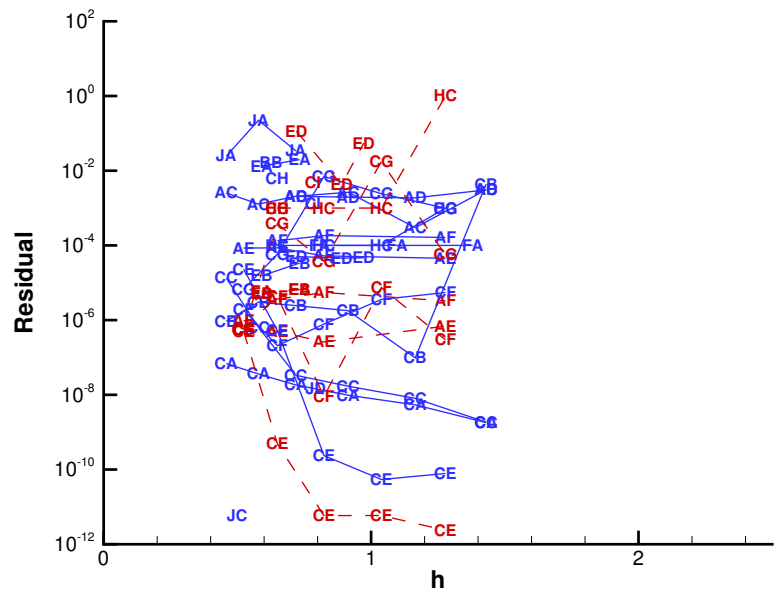

b) Residual.

Figure 32. C25F force and iterative convergence, -Euler, -RANS. 


\section{C25P Submissions}

The submissions for the C25P model are listed in Table 9. Inviscid and viscous grid families with WS-Mixed and WSTet topology are provided for this case as detailed in the Required Grids section. Details of the propulsion boundary conditions are provided in section $\mathrm{C} 25 \mathrm{~F}$ and $\mathrm{C} 25 \mathrm{P}$.

\begin{tabular}{lllll} 
& & \multicolumn{2}{c}{ Table 9. C25P submissions. } \\
Case & Code & Physics & Grid & Millions of Control Volumes $(h)$ \\
\hline CA & FUN3D-VA & Euler & WS-Mixed & $51.9(0.58), 27.1(0.72), 13.2(0.91), 6.4(1.16), 3.4(1.43)$ \\
CB & FUN3D-VA & Euler & WS-Tet & $51.9(0.58), 27.1(0.72), 13.2(0.91), 6.4(1.16), 3.4(1.43)$ \\
CC & FUN3D-VA & RANS-SA & WS-Mixed & $70.6(0.52), 36.9(0.65), 18.1(0.82), 9.1(1.03), 4.9(1.27)$ \\
CD & FUN3D-VA & RANS-SA & WS-Tet & $70.6(0.52), 36.9(0.65), 18.1(0.82), 9.1(1.03), 4.9(1.27)$ \\
CH & FUN3D-VA & RANS-SA & ANSA-Mixed & $39.4(0.63)$ \\
CI & FUN3D-VA & RANS-SA & PW-00001baf-Tet & $23.6(0.75), 24.6(0.74), 24.0(0.75)$ \\
FA & Cart3D & Euler & Adapt-Cart & $34.9(0.66), 11.7(0.95), 6.5(1.15)$ \\
HC & CFD++ & RANS-RKE & WS-Mixed & $18.1(0.82), 9.1(1.03), 4.9(1.27)$ \\
IA & USM3D & Euler & WS-Tet & $37.5(0.64), 20.0(0.79)$ \\
IB & USM3D & RANS-SA & WS-Tet & $53.3(0.57), 28.5(0.71)$ \\
ID & USM3D & RANS-SA & PW-00001baf-Tet & $138.7(0.42), 145.1(0.41)$ \\
KA & Wolf & Euler & WS-Tet & $27.1(0.72), 13.2(0.91), 6.4(1.16), 3.4(1.43)$ \\
KB & Wolf-V6 & Euler & WS-Tet & $27.1(0.72), 13.2(0.91), 6.4(1.16), 3.4(1.43)$ \\
KC & Wolf & RANS-SA & WS-Tet & $18.1(0.82), 9.1(1.03), 4.9(1.27)$ \\
KD & Wolf-V6 & RANS-SA & WS-Tet & $18.1(0.82)$
\end{tabular}

The nearfield ensemble mean and standard deviation are shown in Fig. 33 near the centerline, Fig. 34 at intermediate offtrack angles, and Fig. 35 at large offtrack angles. This model has 6 Euler and 9 RANS fine grid submission sample sizes. The differences in the Euler and RANS means is largest near the centerline and persists at all extraction distances. The difference between the means of the two physical modeling approaches is larger than the standard deviation of either group, particularly in the range of TAU $=[0.7-1.0]$ and the aft signature influenced by the plume.

Figure 36 shows selected signatures at $\mathrm{PHI}=0^{\circ}$ and $\mathrm{PHI}=20^{\circ}$, respectively, extracted from the inviscid simulations at $\mathrm{R}=5$. $\mathrm{PHI}=20^{\circ}$ was selected based on the PL results, which are presented later in this section. The number of control volumes in the grids used to plot the selected signatures is noted in the captions. Cases CA, CB and KA predict very similar signatures. We show case $\mathrm{CA}$ at $\mathrm{PHI}=0^{\circ}$ and $\mathrm{PHI}=20^{\circ}$ in Figs. $36 \mathrm{a}$ and $36 \mathrm{~b}$, respectively, as an example. On-track, case FA (Fig. 36c) and KB (Fig. 36e) predict a shallower expansion near TAU=0.95 when compared to case CA. Note that the error estimates in this region indicate moderate sensitivity to mesh refinement. Signatures predicted by KB contain numerous small pressure fluctuations that appear to be increasing in amplitude with grid refinement. At $\mathrm{PHI}=20^{\circ}$ (right side of Fig. 36), there is excellent agreement among the signatures with only small deviations in the expansion near TAU $=1.1$ and the pressure fluctuations in the wake region.

Figure 37 shows selected signatures at $\mathrm{PHI}=0^{\circ}$ and $\mathrm{PHI}=20^{\circ}$, respectively, extracted from the viscous simulations at $\mathrm{R}=5$. Signatures from cases $\mathrm{CC}$ and $\mathrm{CD}$ are very similar - we show signatures from CC in Figs. 37a and 37b. Despite the coarser mesh used in case HC, Figs. $37 \mathrm{c}$ and $37 \mathrm{~d}$ show that the HC signatures match closely those from CC. The largest pressure differences and error estimates are in the wake region, especially at $\mathrm{PHI}=20^{\circ}$. Signatures from case $\mathrm{KC}$ are not as well resolved, and the error analysis reveals that the level of error is increasing with grid refinement. Hence, the error estimate is marked "Low Confidence" in Figs. 37e and 37f.

The PL grid convergence of the submissions is shown in Fig. 38 near the centerline, Fig. 39 at intermediate offtrack angles, and Fig. 40 at large offtrack angles. The Euler submissions are shown in blue and the RANS submissions are shown in red. The agreement between Euler and RANS physical models is better for the C25P than the C25F at $\mathrm{PHI}=0^{\circ}$ and $10^{\circ}$. The Euler Case IA is quieter then those from other participants at one body length, but in agreement with the group at larger body lengths. The single grid $\mathrm{CH}$ is louder than other RANS cases beyond one body length. The trend of louder $\mathrm{CH}$ case continues at a $\mathrm{PHI}=20^{\circ}$. Without a grid refinement sequence, no trends can be formed for $\mathrm{CH}$. The variation in the RANS submissions is larger than the Euler submissions at PHI of $20^{\circ}$ and $30^{\circ}$ at one body length. The larger spread and trend reversal in $\mathrm{PL}$ at $\mathrm{PHI}=20^{\circ}$ (Fig. 39e) versus PHI $=0^{\circ}$ (Fig. 38e) could be due to the deeper expansions of the RANS signatures shown in Figs. 36 and 37. The Euler and RANS appear to converge to similar values for PHI of $40^{\circ}$ and $50^{\circ}$. The Euler submissions appear to converge to a lower PL value at $50^{\circ} \mathrm{PHI}$ but in a less pronounced manner than the $\mathrm{C} 25 \mathrm{~F}$.

Box, whisker, and violin plots of PL calculated from the fine grid Euler and RANS submissions are shown in Fig. 41. The PL values are plotted for each offtrack angle to produce PL carpet plots with submissions provided at one, three, and five body lengths. The interquartile range (box extent) is larger for the RANS submissions at $10^{\circ}-$ $20^{\circ} \mathrm{PHI}$, which is also observed in Fig. 39. A common trend with the $\mathrm{C} 25 \mathrm{~F}$ is more outliers for the RANS physical model than the Euler physical model and larger interquartile ranges. Case IA was a low outlier, and case KB was a 
high outlier for some Euler extraction locations. Cases $\mathrm{CH}, \mathrm{HC}$, and $\mathrm{KD}$ were high outliers and $\mathrm{KD}$ was a low outlier for some RANS extraction locations.

The convergence of lift and relative iterative convergence for the $\mathrm{C} 25 \mathrm{P}$ submissions is shown in Fig. 42. The Euler submissions trend toward a higher lift value than the RANS submissions, which is a common trend with the C25F. The relative meanflow equation iterative convergence is shown in blue solid lines and the turbulence model relative convergence is shown in red with dashed lines. The iterative convergence generally worsened with increasing grid refinement. Case CA had a dramatic reduction in iterative convergence on the finest grid. 


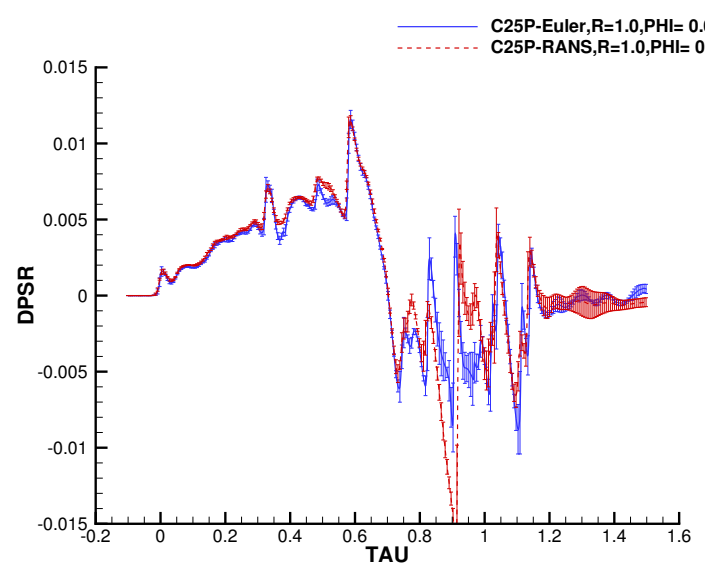

a) $\mathrm{R}=\mathbf{1}, \mathbf{P H I}=\mathbf{0}^{\circ}$.

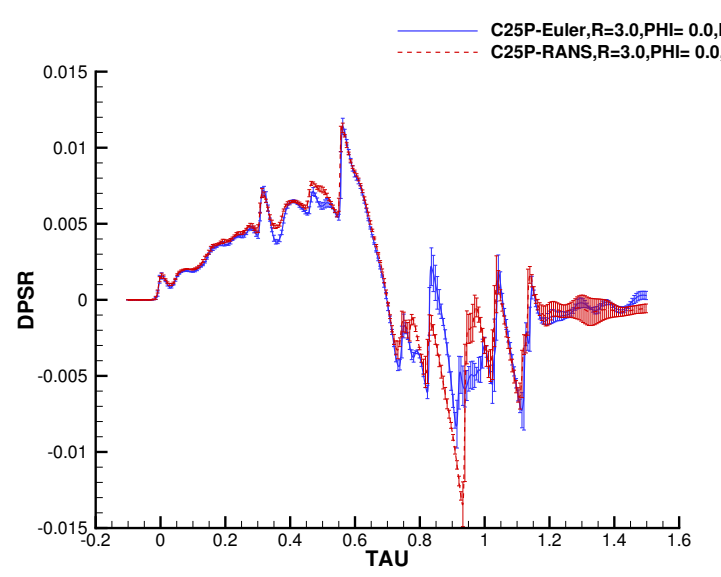

c) $\mathrm{R}=3, \mathrm{PHI}=\mathbf{0}^{\circ}$.

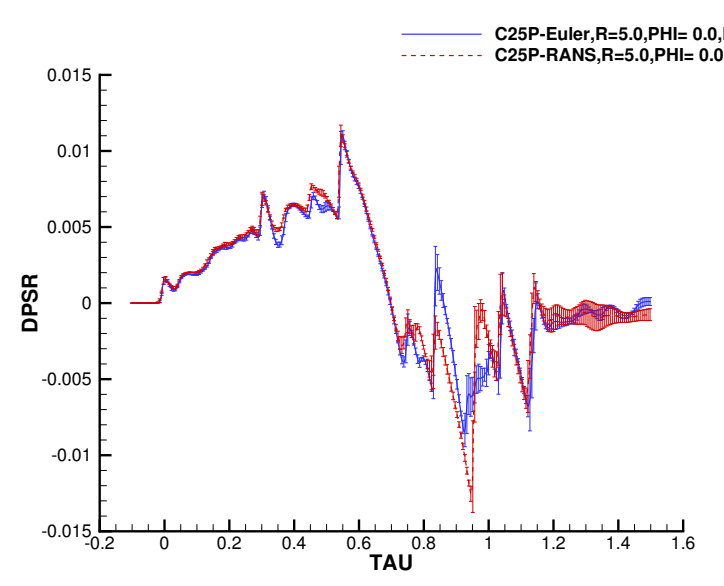

e) $\mathrm{R}=5$, $\mathrm{PHI}=0^{\circ}$.

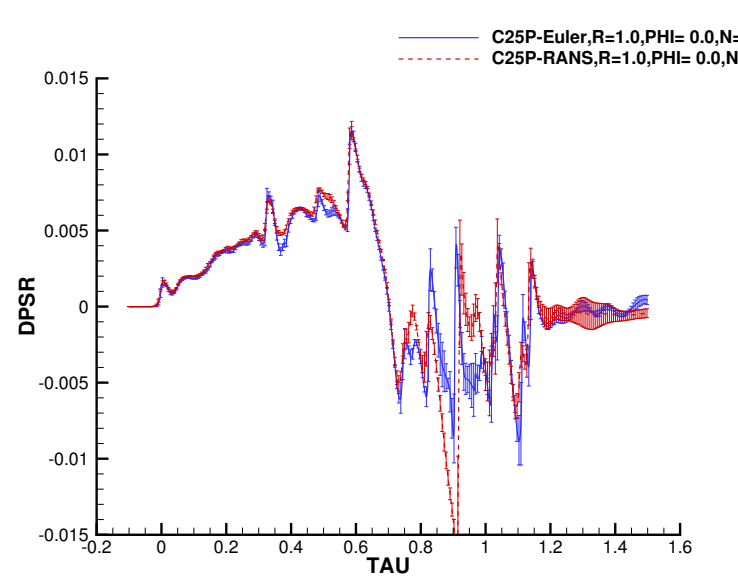

b) $\mathrm{R}=1, \mathrm{PHI}=10^{\circ}$.

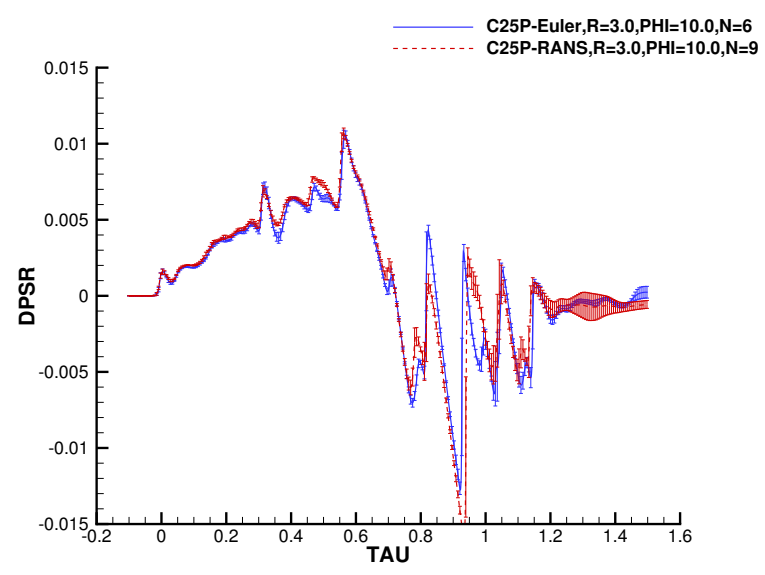

d) $\mathrm{R}=3, \mathrm{PHI}=10^{\circ}$.

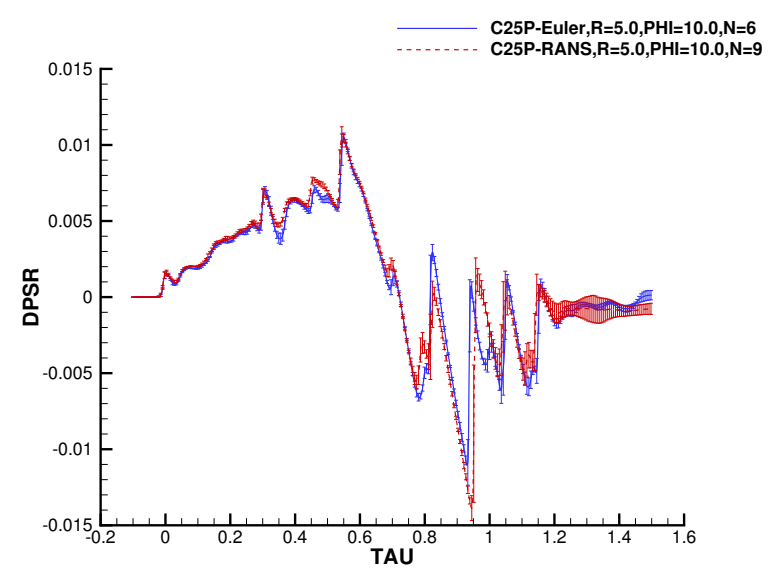

f) $\mathrm{R}=5, \mathrm{PHI}=10^{\circ}$.

Figure 33. $\mathrm{C25P}$ nearfield ensemble mean and standard deviation for fine grids, $\mathrm{PHI}=0^{\circ}-10^{\circ}$. 


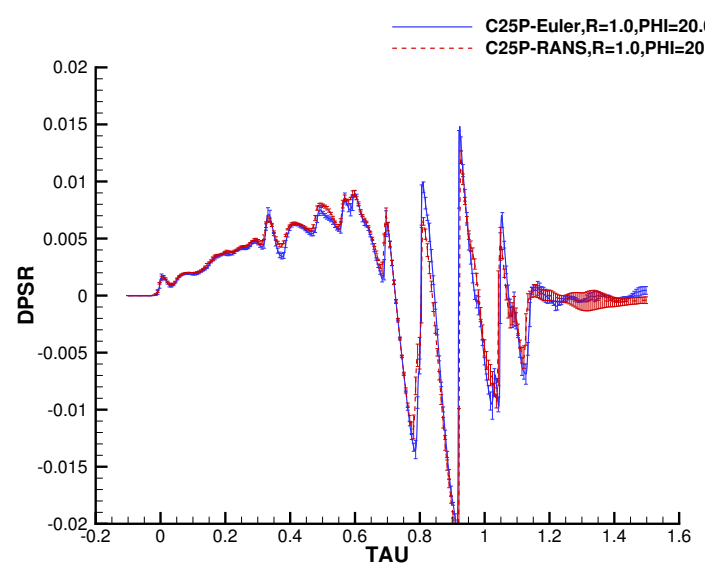

a) $\mathbf{R = 1}, \mathbf{P H I}=\mathbf{2 0}{ }^{\circ}$.

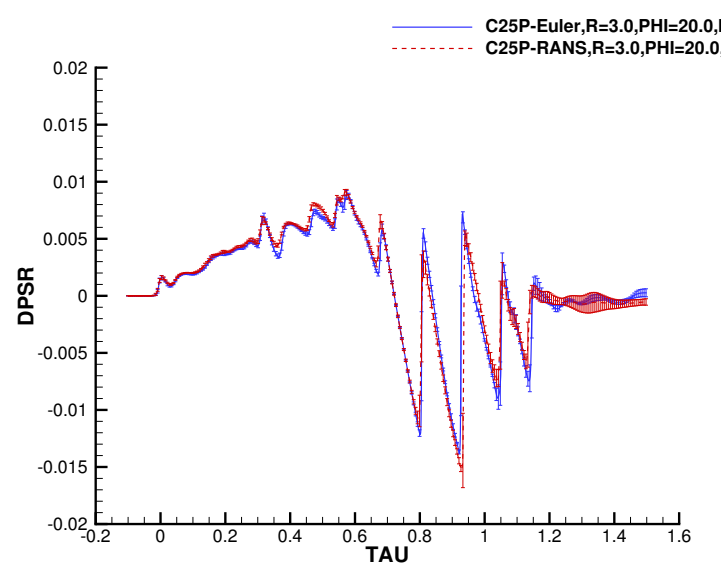

c) $\mathrm{R}=3, \mathrm{PHI}=20^{\circ}$.

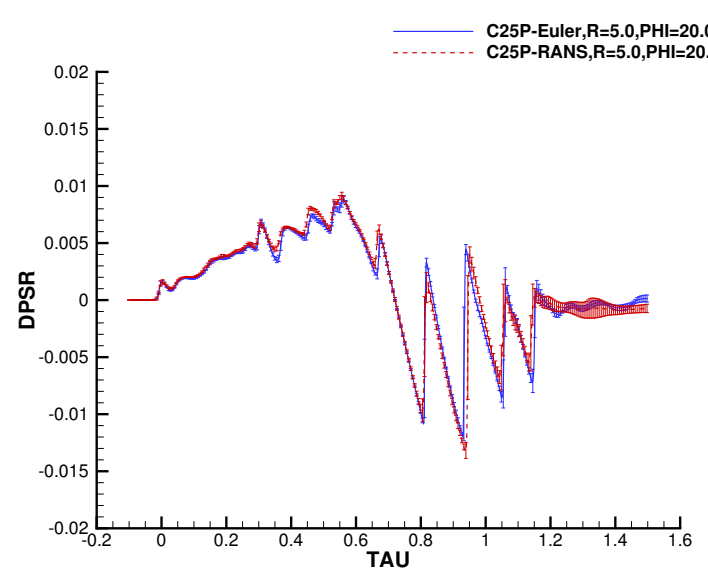

e) $\mathbf{R}=5$, $\mathrm{PHI}=20^{\circ}$.

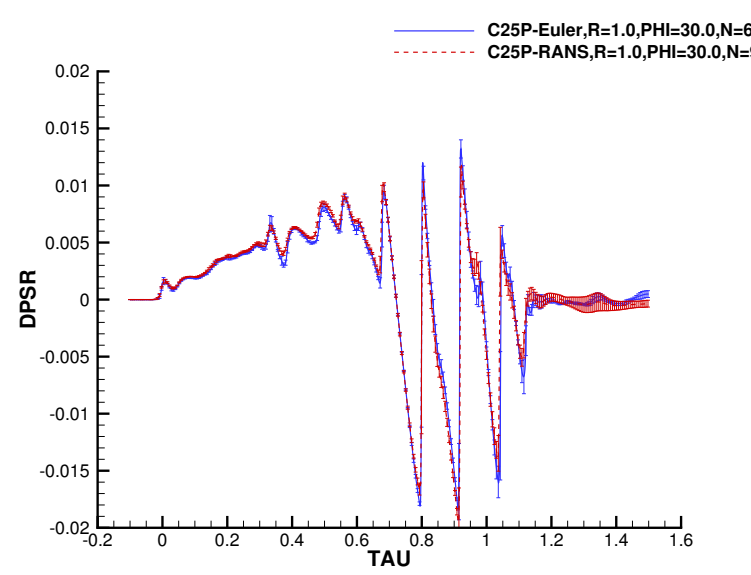

b) $\mathrm{R}=1, \mathrm{PHI}=30^{\circ}$.

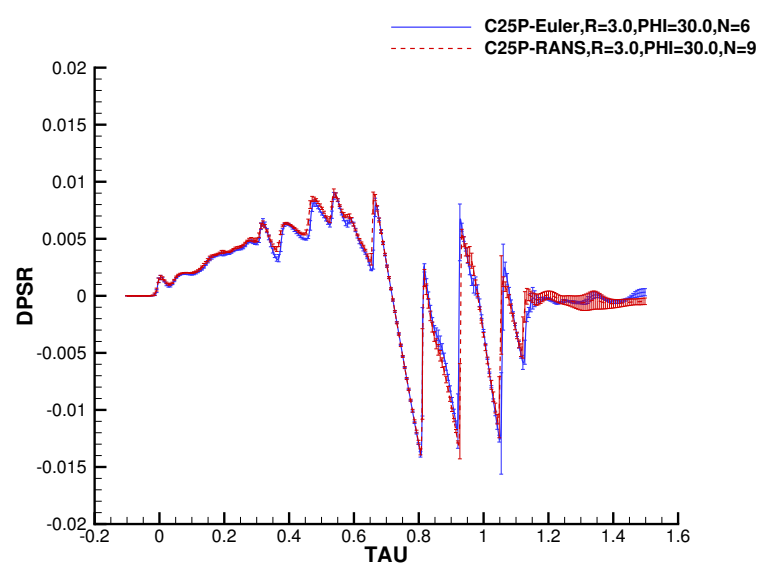

d) $\mathrm{R}=3, \mathrm{PHI}=30^{\circ}$.

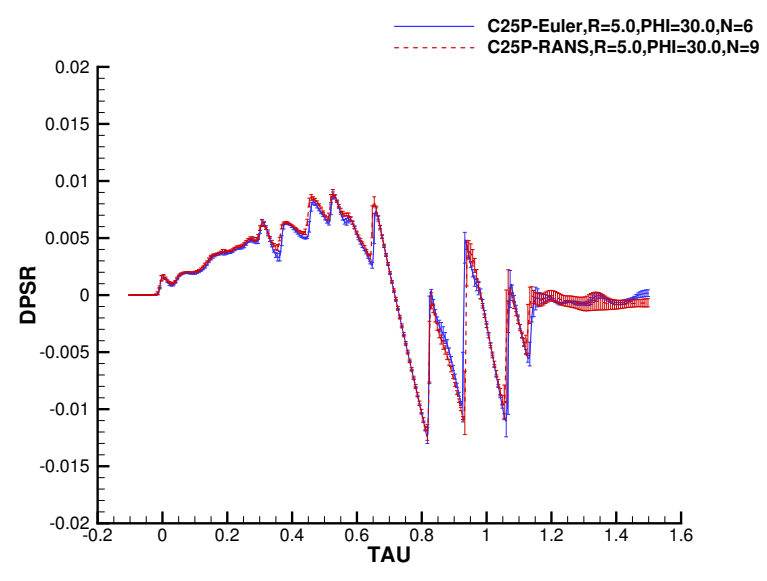

f) $\mathrm{R}=5, \mathrm{PHI}=30^{\circ}$.

Figure 34. $\mathrm{C25P}$ nearfield ensemble mean and standard deviation for fine grids, $\mathrm{PHI}=20^{\circ}-30^{\circ}$. 


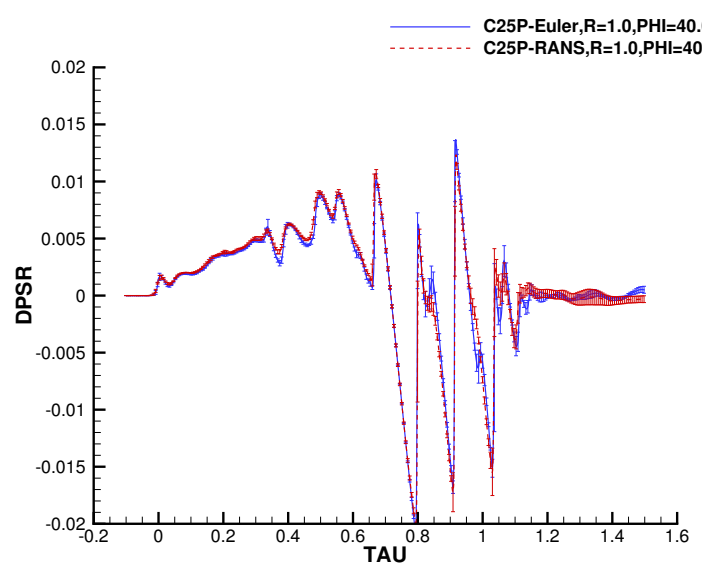

a) $\mathrm{R}=\mathbf{1}, \mathbf{P H I}=\mathbf{4 0}^{\circ}$.

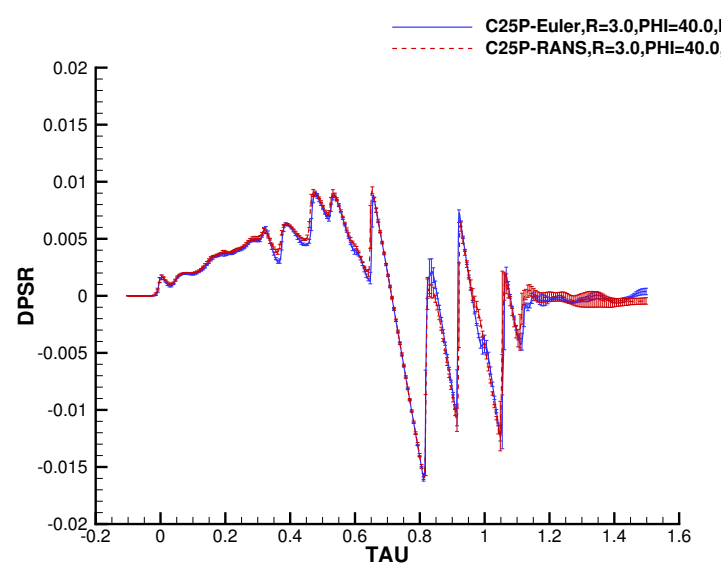

c) $\mathrm{R}=3, \mathrm{PHI}=40^{\circ}$.

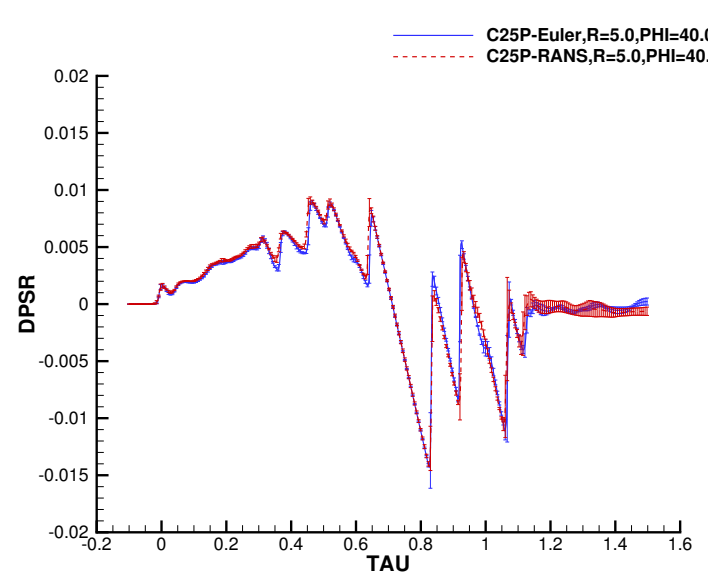

e) $\mathrm{R}=5$, $\mathrm{PHI}=\mathbf{4 0}$.

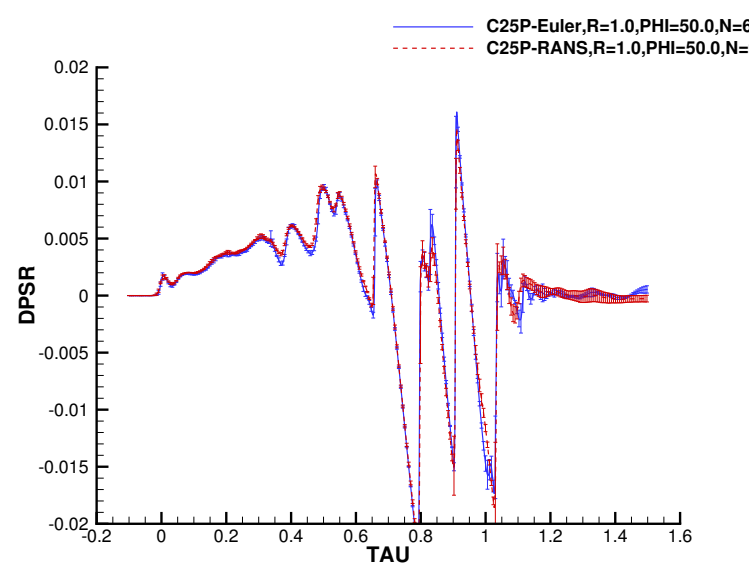

b) $\mathrm{R}=1, \mathrm{PHI}=50^{\circ}$.

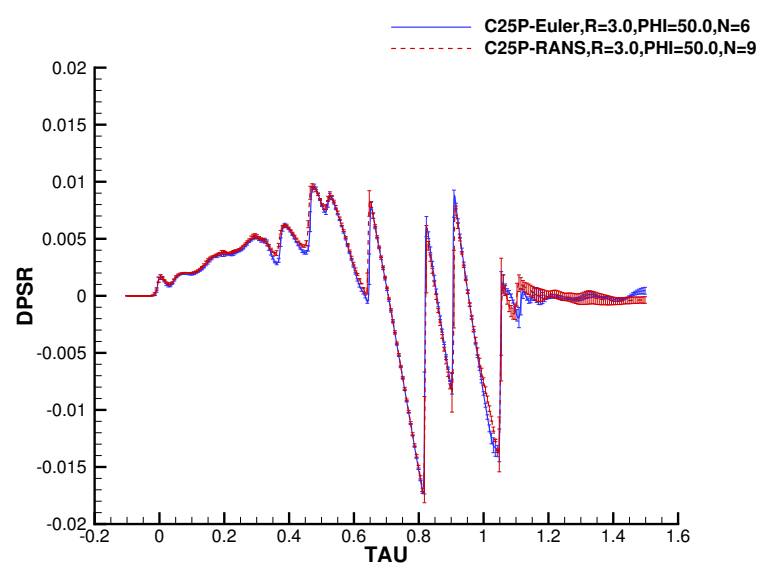

d) $\mathrm{R}=3, \mathrm{PHI}=50^{\circ}$.

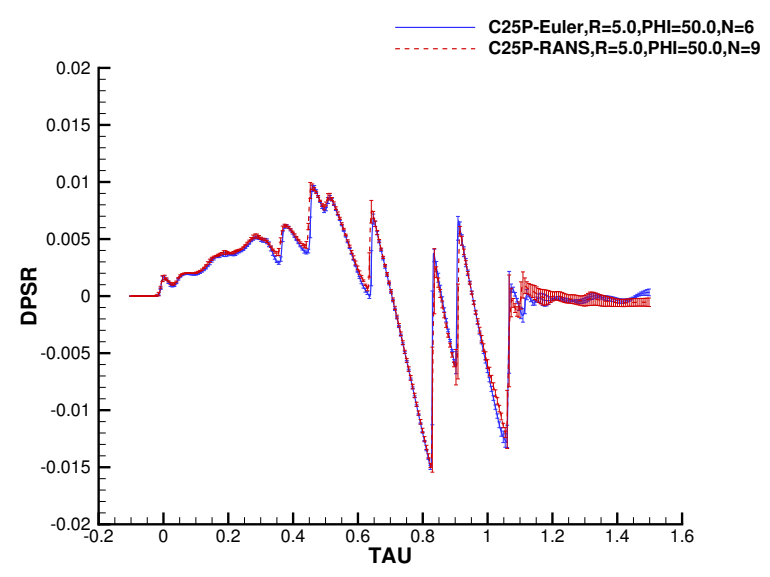

f) $\mathbf{R}=5, \mathrm{PHI}=50^{\circ}$.

Figure 35. $\mathrm{C25P}$ nearfield ensemble mean and standard deviation for fine grids, $\mathrm{PHI}=40^{\circ}-50^{\circ}$. 


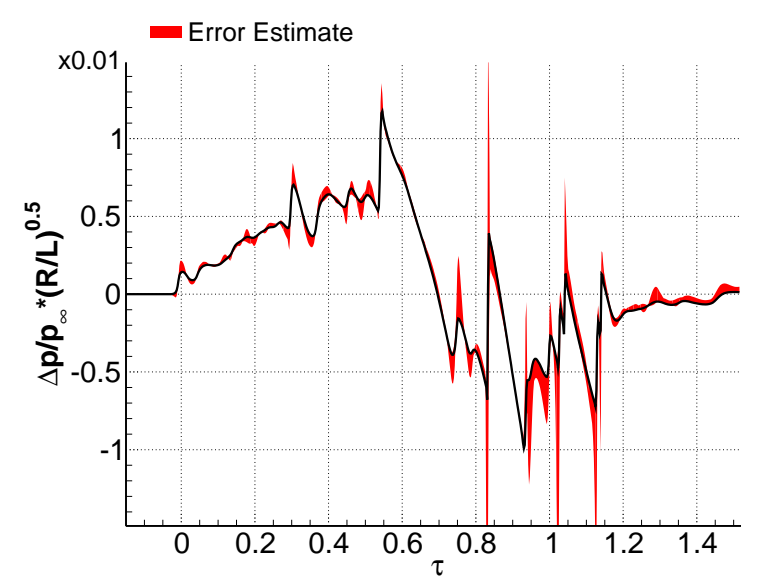

a) $\mathrm{PHI}=0^{\circ}$ : $\mathrm{CA}($ shown, 51.9M), CB, KA.

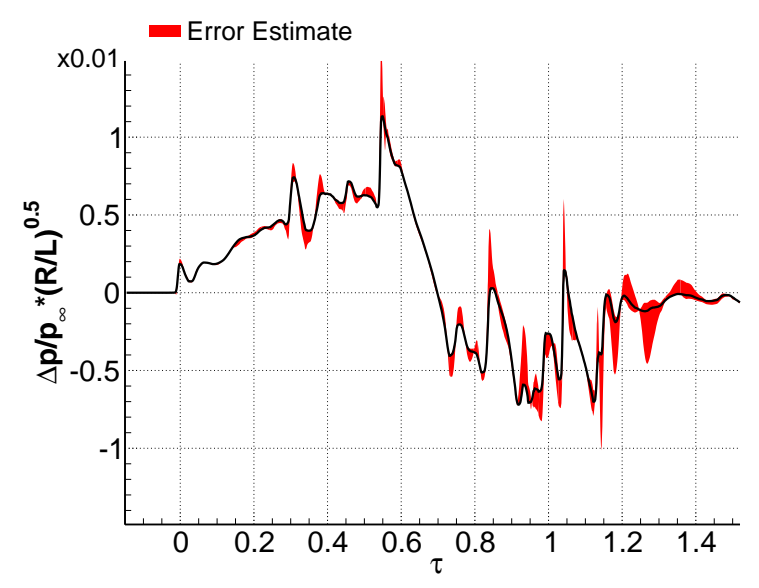

c) $\mathrm{PHI}=0^{\circ}$ : FA (34.9M).

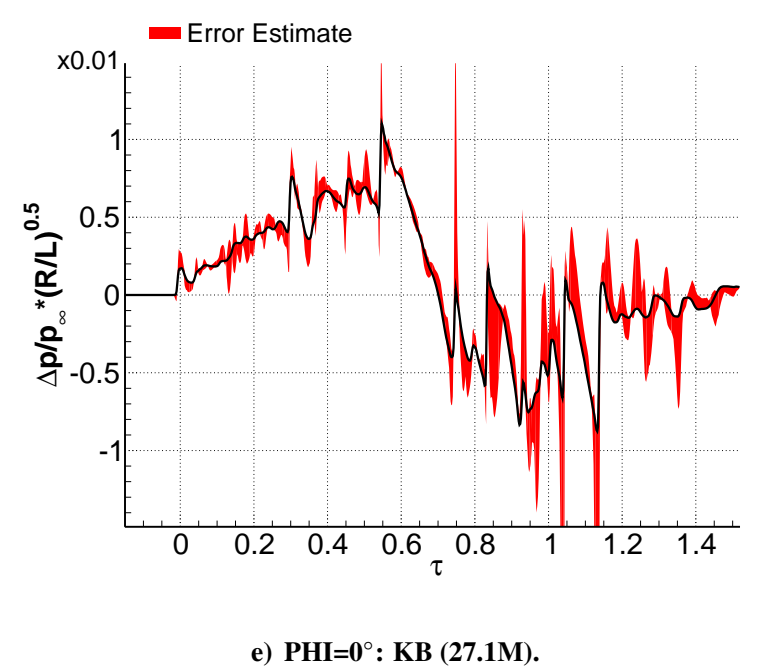

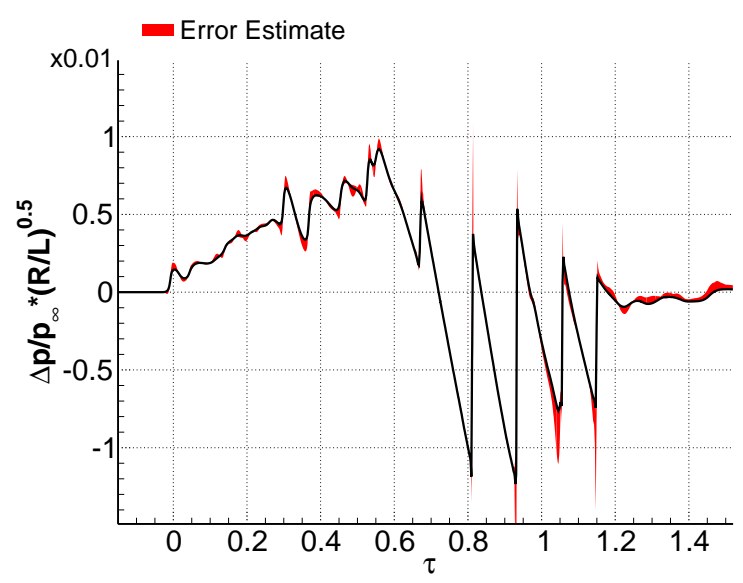

b) $\mathrm{PHI}=20^{\circ}$ : CA (shown, 51.9M), CB, KA.

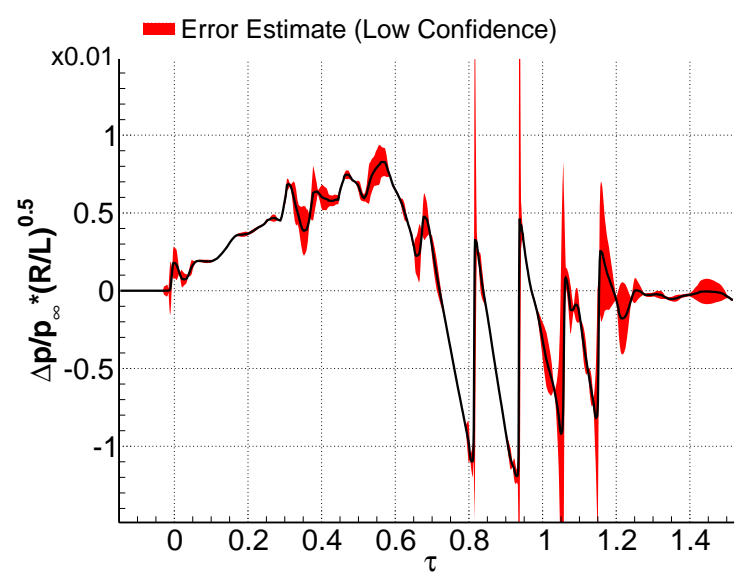

d) $\mathrm{PHI}=20^{\circ}$ : $\mathrm{FA}(34.9 \mathrm{M})$.

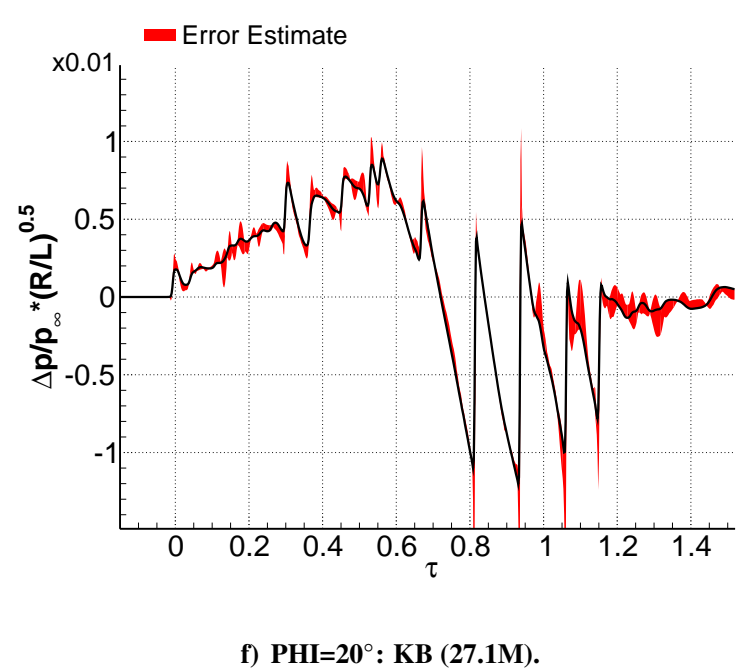

Figure 36. $\mathrm{C25P}$ Euler signatures computed on fine grids with discretization error estimates: $\mathrm{R}=5, \mathrm{PHI}=0^{\circ}(l \mathrm{eft})$ and $\mathrm{PHI}=20^{\circ}($ right $)$. 


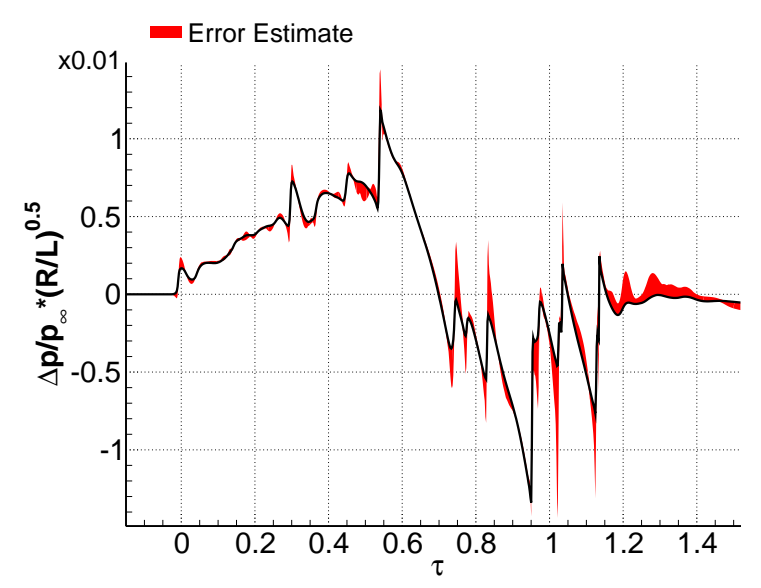

a) $\mathrm{PHI}=0^{\circ}$ : $\mathrm{CC}(\mathbf{7 0 . 6 M})$.

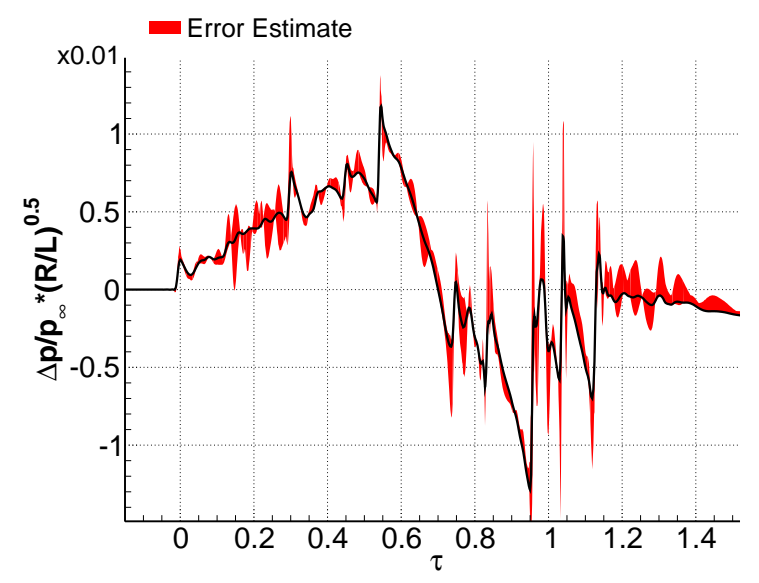

c) $\mathrm{PHI}=0^{\circ}: \mathrm{HC}(18.1 \mathrm{M})$.

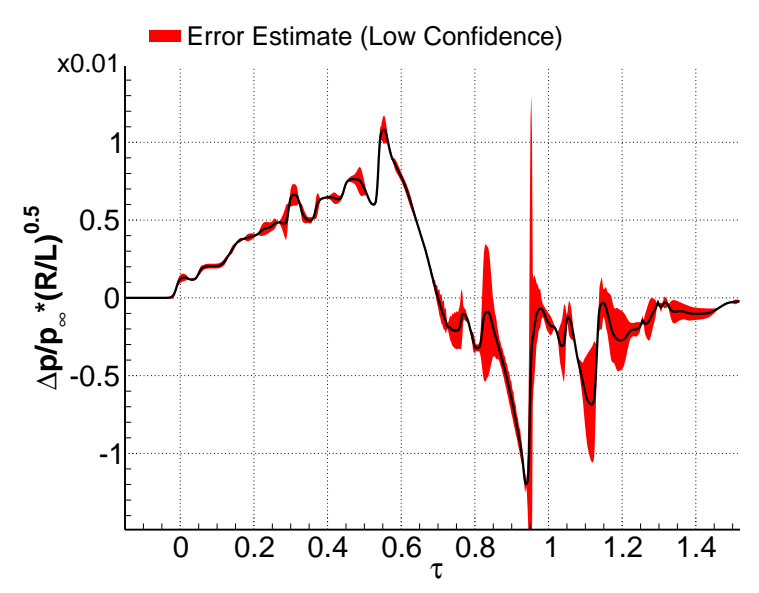

e) $\mathrm{PHI}=0^{\circ}: \mathrm{KC}(18.1 \mathrm{M})$.

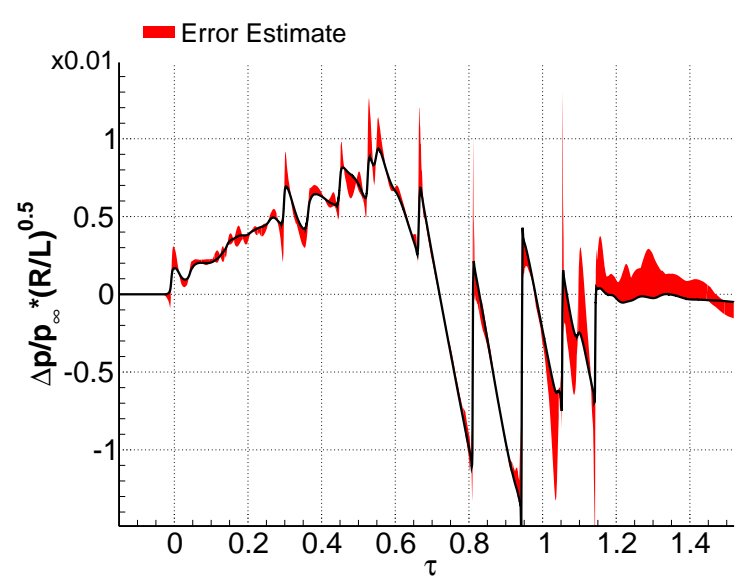

b) $\mathrm{PHI}=20^{\circ}$ : $\mathrm{CC}(70.6 \mathrm{M})$.

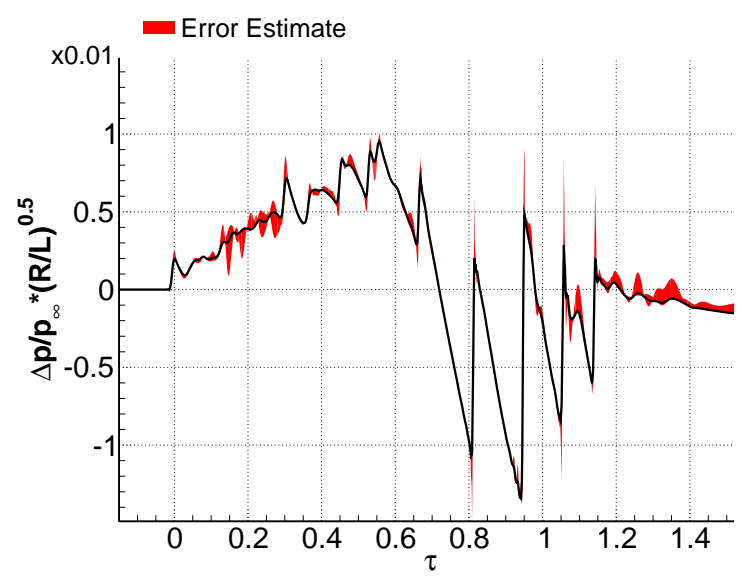

d) $\mathrm{PHI}=20^{\circ}: \mathrm{HC}(18.1 \mathrm{M})$.

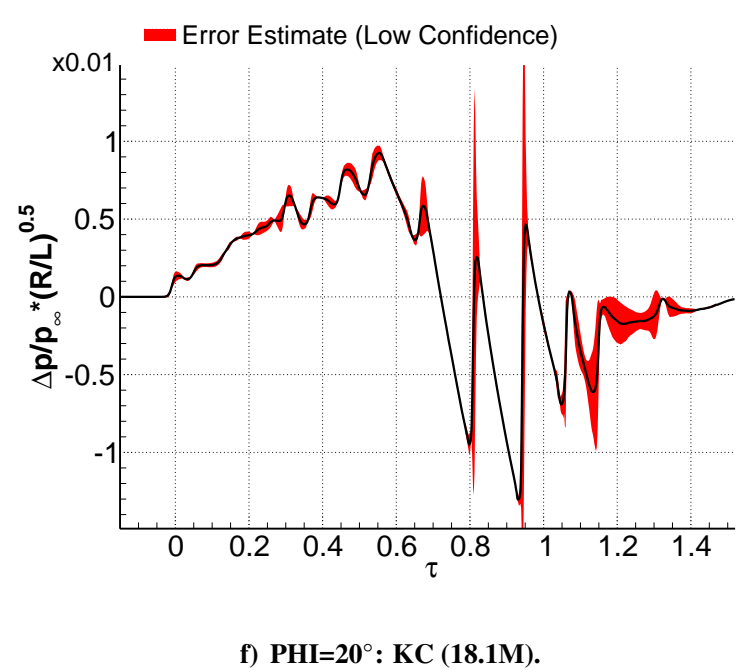

Figure 37. C25P RANS signatures computed on fine grids with discretization error estimates: $\mathrm{R}=5, \mathrm{PHI}=0^{\circ}($ left $)$ and $\mathrm{PHI}=20^{\circ}($ right $)$. 


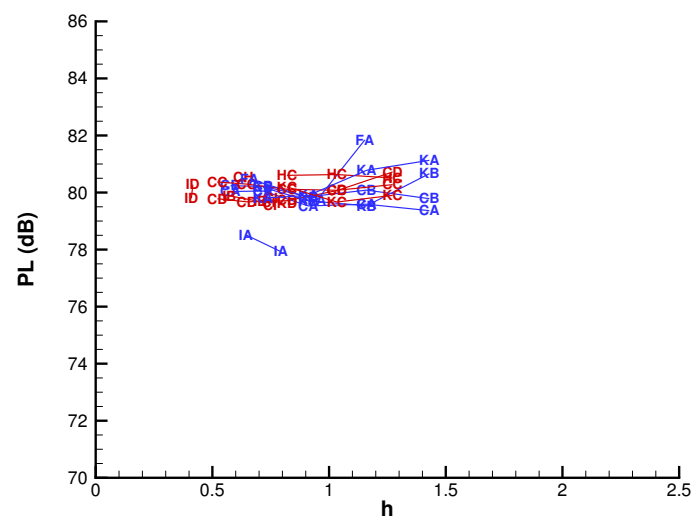

a) $\mathrm{R}=\mathbf{1}, \mathbf{P H I}=0^{\circ}$.

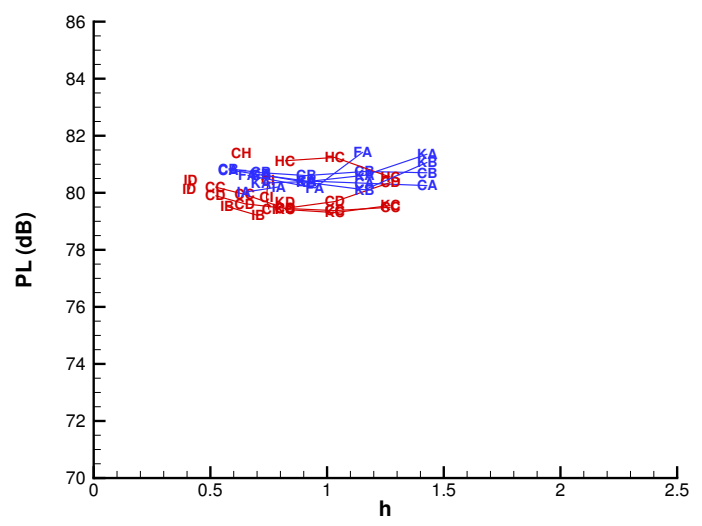

c) $\mathrm{R}=3, \mathrm{PHI}=0^{\circ}$.

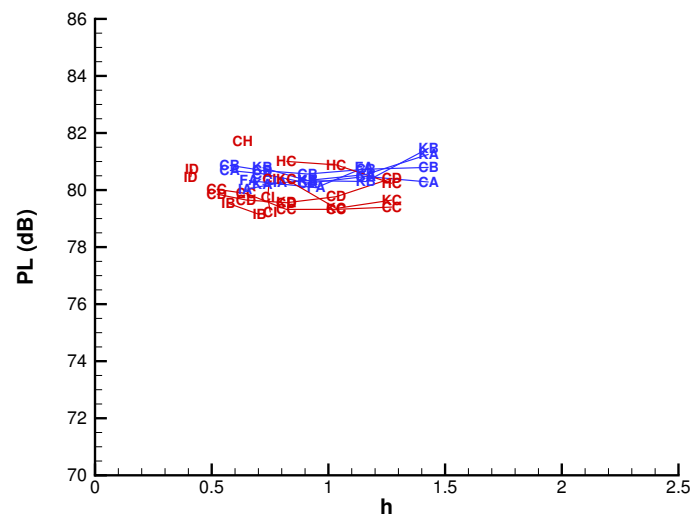

e) $\mathrm{R}=5$, $\mathrm{PHI}=0^{\circ}$.

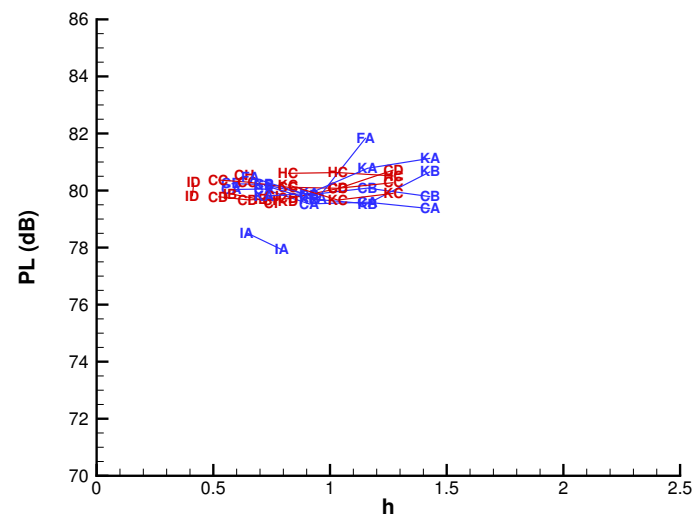

b) $\mathrm{R}=1, \mathrm{PHI}=10^{\circ}$.

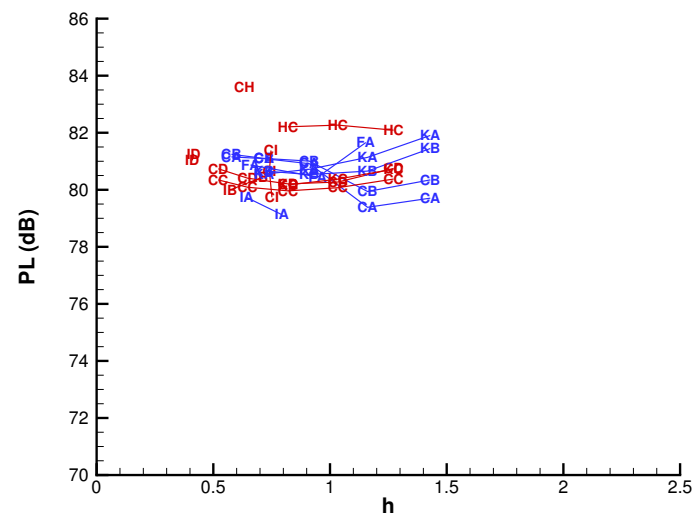

d) $\mathrm{R}=3, \mathrm{PHI}=10^{\circ}$.

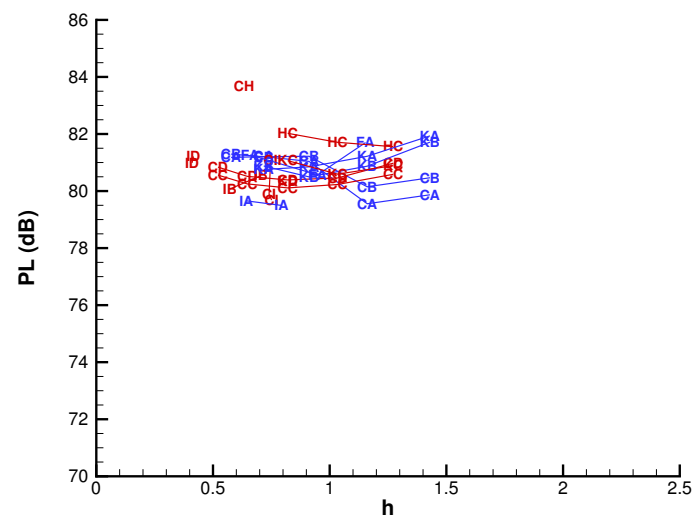

f) $\mathrm{R}=5, \mathrm{PHI}=10^{\circ}$.

Figure 38. C25P PL grid convergence, $\mathrm{PHI}=0^{\circ}-10^{\circ}$, -Euler, - RANS. 


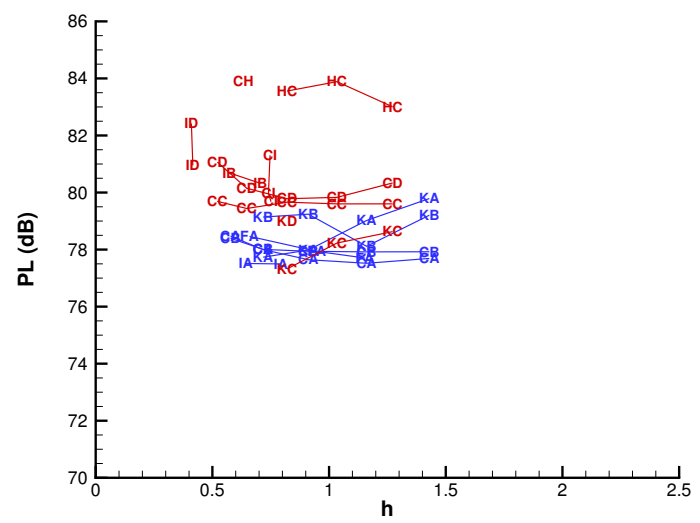

a) $\mathrm{R}=\mathbf{1}, \mathrm{PHI}=\mathbf{2 0}{ }^{\circ}$.

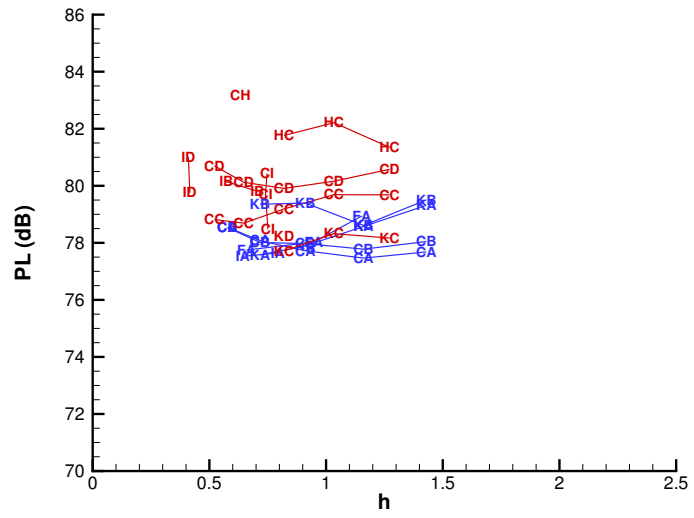

c) $\mathrm{R}=3, \mathrm{PHI}=20^{\circ}$

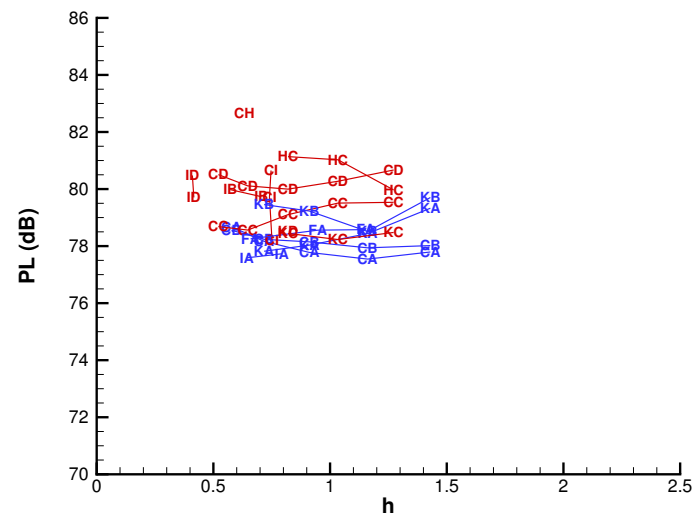

e) $\mathrm{R}=5, \mathrm{PHI}=20^{\circ}$.

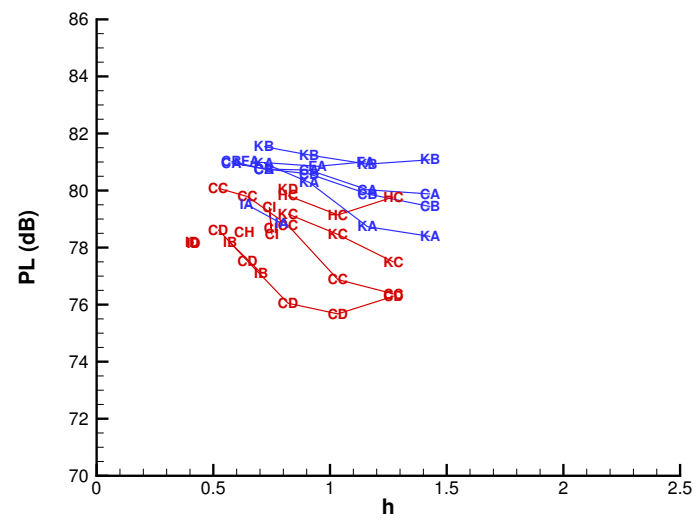

b) $\mathrm{R}=1, \mathrm{PHI}=30^{\circ}$.

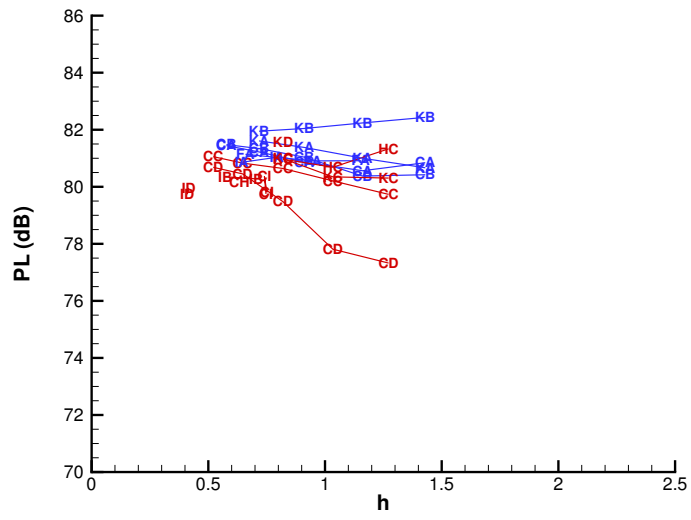

d) $\mathbf{R}=3, \mathbf{P H I}=30^{\circ}$.

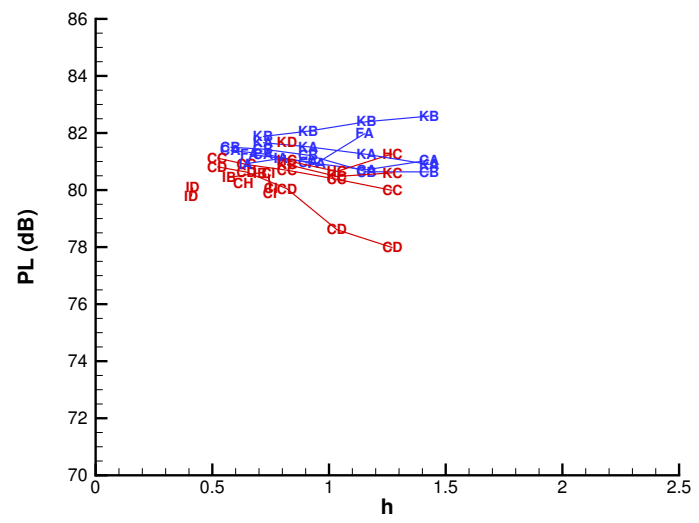

f) $\mathrm{R}=5, \mathrm{PHI}=30^{\circ}$.

Figure 39. C25P PL grid convergence, $\mathrm{PHI}=20^{\circ}-30^{\circ}$, -Euler, -RANS. 


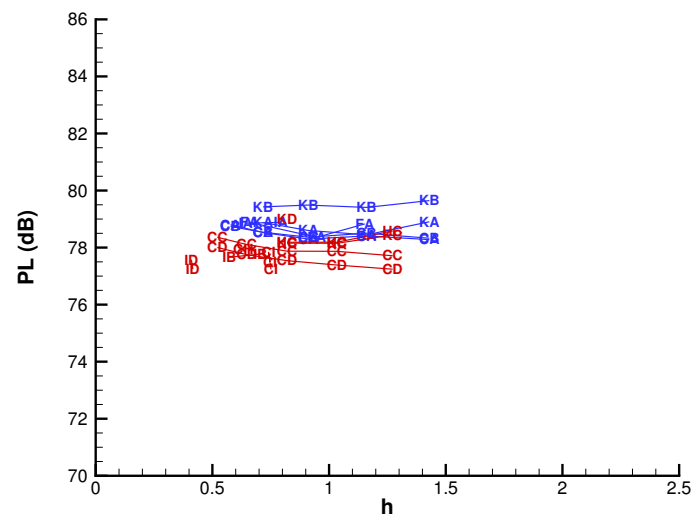

a) $\mathrm{R}=\mathbf{1}, \mathrm{PHI}=40^{\circ}$.

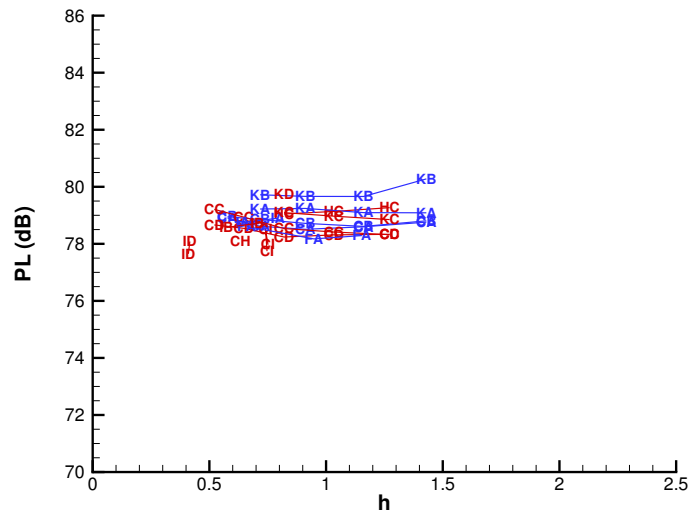

c) $\mathbf{R}=3, \mathrm{PHI}=40^{\circ}$.

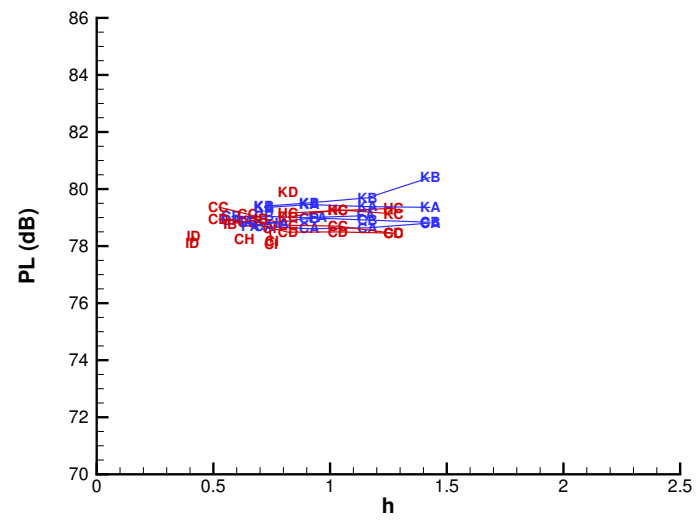

e) $\mathrm{R}=5$, $\mathrm{PHI}=40^{\circ}$.

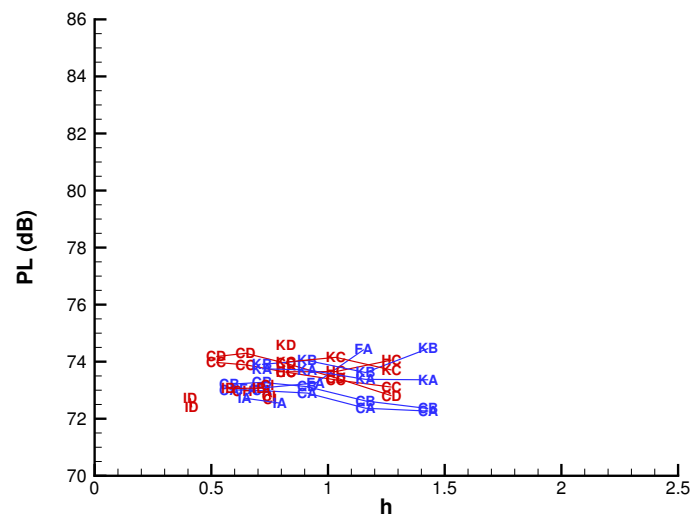

b) $\mathrm{R}=\mathbf{1}, \mathrm{PHI}=\mathbf{5 0}$.

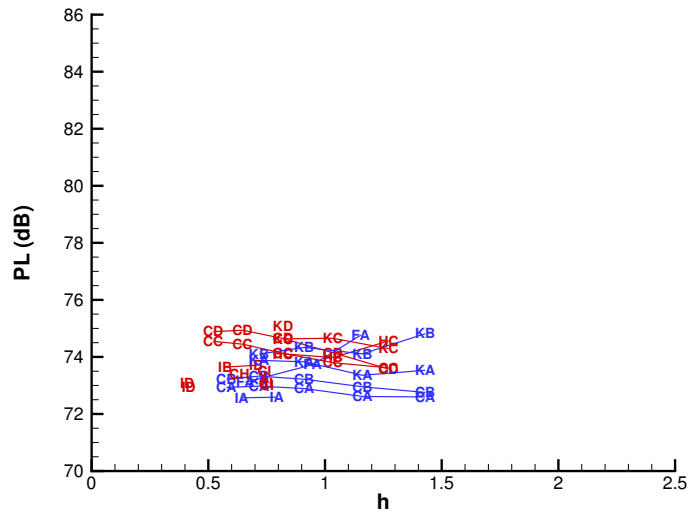

d) $\mathbf{R = 3}, \mathrm{PHI}=50^{\circ}$.

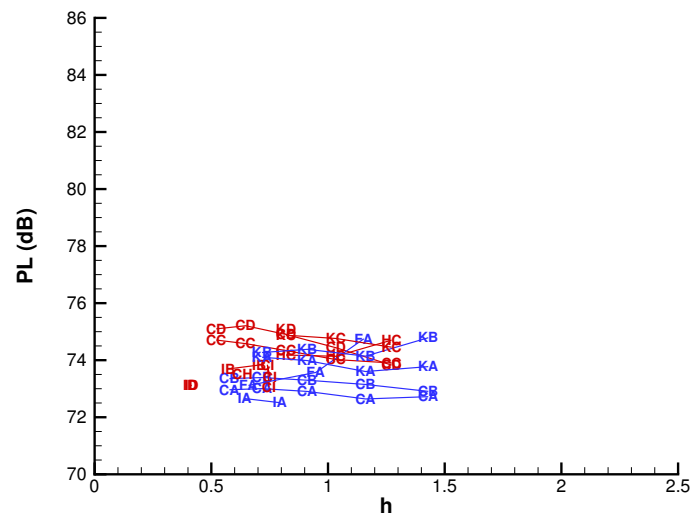

f) $\mathrm{R}=5, \mathrm{PHI}=50^{\circ}$.

Figure 40. C25P PL grid convergence, $\mathrm{PHI}=40^{\circ}-50^{\circ}$, -Euler, -RANS. 


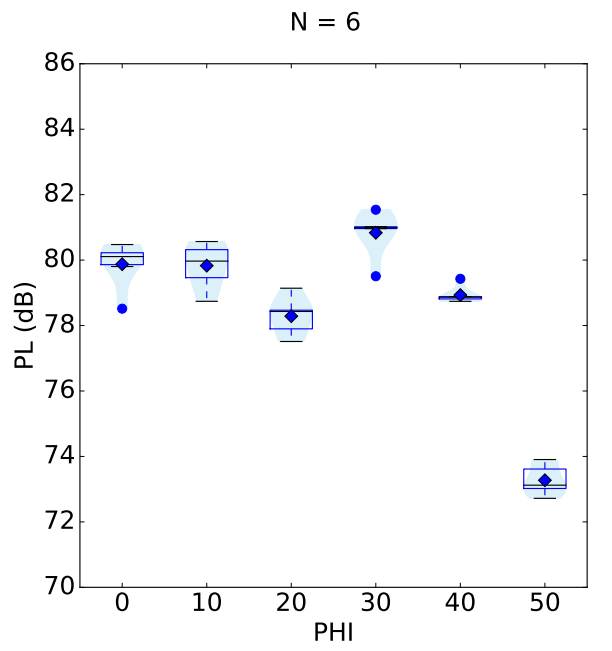

a) Euler $R=1$. $P H I=0^{\circ}$ : IA low. $P H I=30^{\circ}$ : IA low. $\mathrm{PHI}=\mathbf{4 0}^{\circ}$ : KB high.

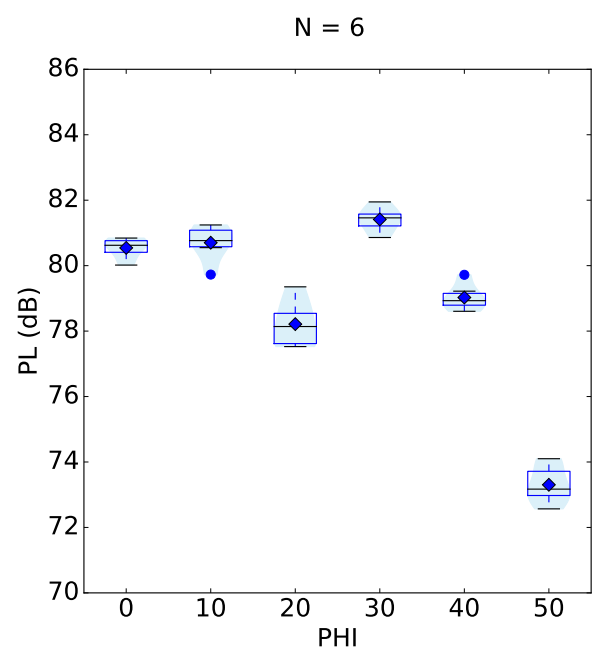

c) Euler $\mathrm{R}=3 . \mathrm{PHI}=10^{\circ}$ : $\mathrm{IA}$ low. $\mathrm{PHI}=40^{\circ}$ : $\mathrm{KB}$ high.

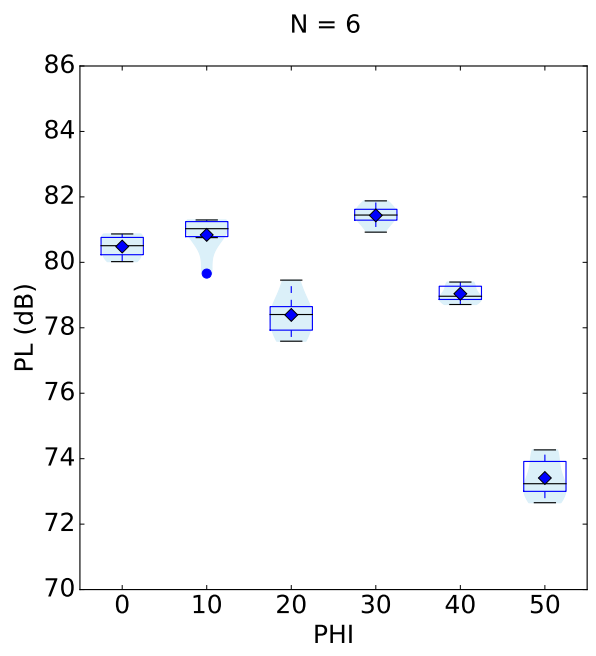

e) Euler $\mathrm{R}=5$. $\mathrm{PHI}=10^{\circ}$ : IA low.

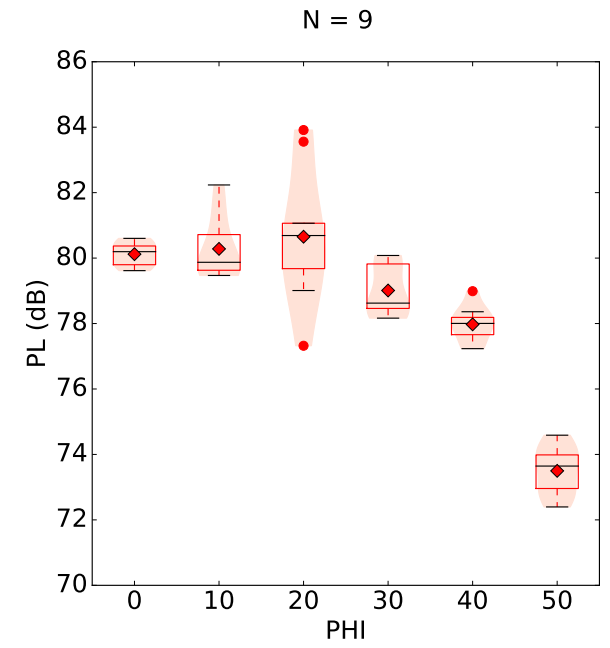

b) RANS R=1. $\mathrm{PHI}=20^{\circ}$ : $\mathrm{CH}, \mathrm{HC}$ high, $\mathrm{KC}$ low. $\mathrm{PHI}=\mathbf{4 0}^{\circ}$ : KD high.

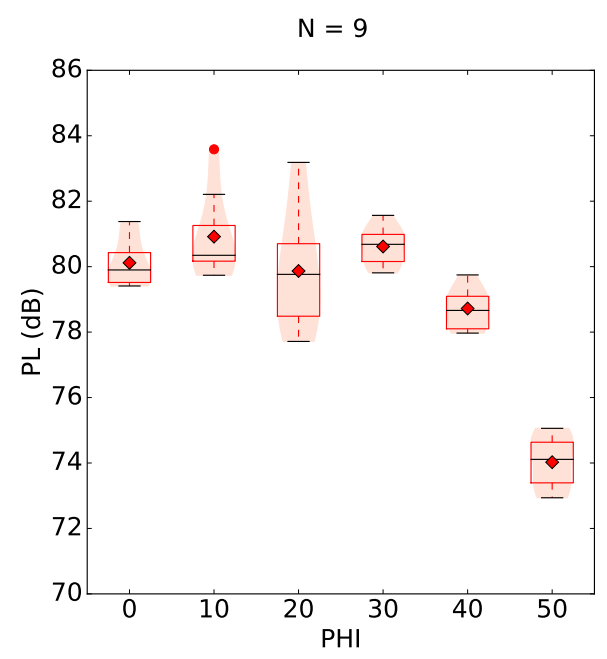

d) RANS R=3. $\mathrm{PHI}=10^{\circ}$ : $\mathrm{CH}$ high.

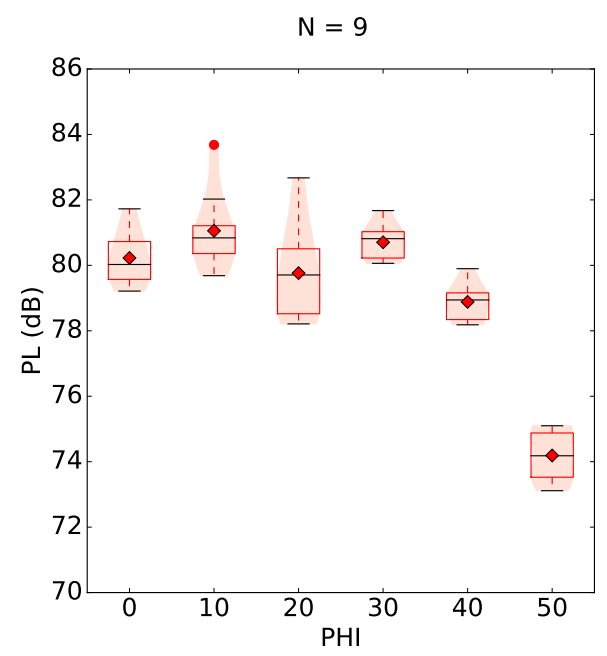

f) RANS R=5. $\mathrm{PHI}=10^{\circ}$ : $\mathrm{CH}$ high.

Figure 41. C25P PL fine grid statistics. 


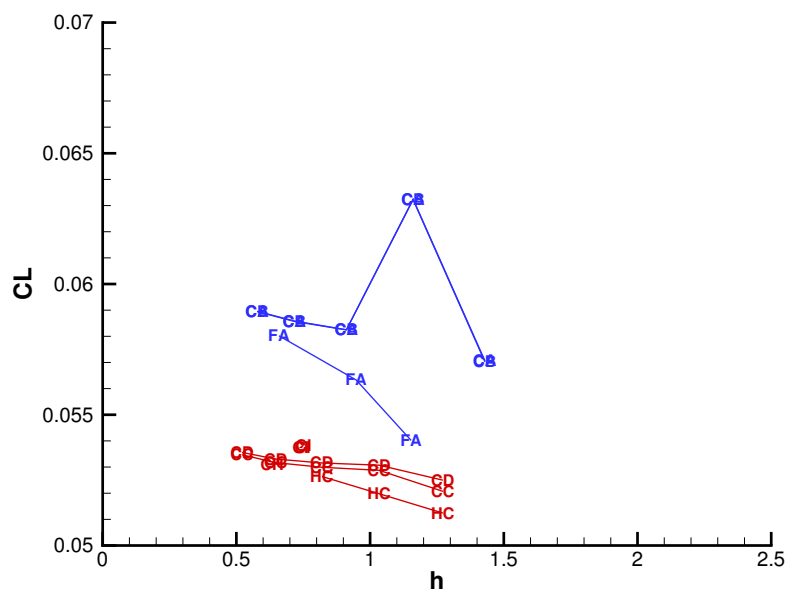

a) Lift Coefficient.

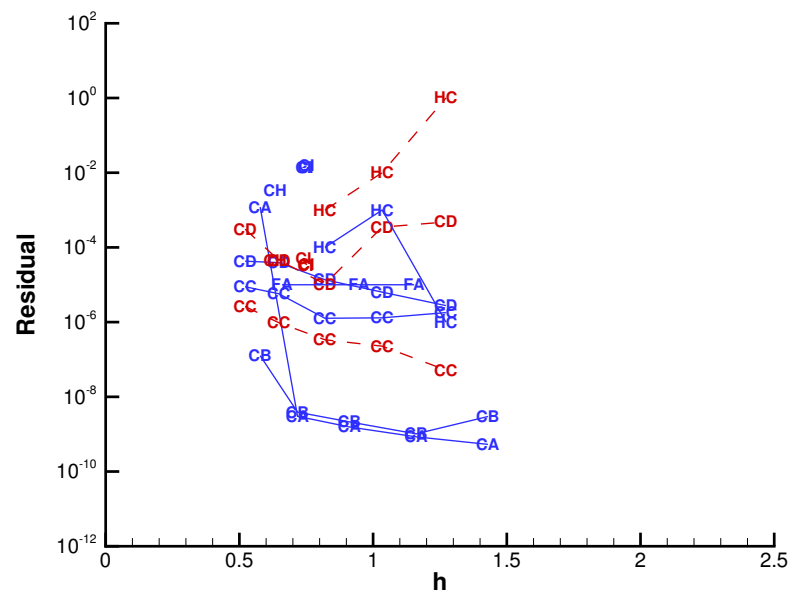

b) Residual.

Figure 42. C25P force and iterative convergence, -Euler, -RANS. 


\section{Participant Presentations}

During SBPW2 each participant group presented details on their experiences performing test cases. These presentations are available on the workshop FTP server and often provide details of investigations that did not result in full submissions. Evidence from these investigations contribute to understanding some of the issues identified in the analysis of submissions.

Participant A plotted the symmetry plane solution in R and TAU coordinates, which clearly illustrates the merging of shocks with expansions and the impact of farfield boundary condition reflections. The least-square gradient reconstruction method in DLR-TAU produced nonphysical solutions on purely tetrahedral grids (JWB). These spurious oscillations were suppressed with a Green-Gauss gradient reconstruction method. Backup slides examined the shocks produced by triangular coarse surface grids that under-resolved the conic nose geometry. Plotting a constant radius extraction surface showed scalloping of the signatures due to polyhedral core-collar grid interface.

Participant B used the WS-Mixed for the AXIE and only custom grids for the more complex cases. Their presentation contains details of these custom viscous grids. Participant $C$ showed iterative convergence issues in the aft large radius section of the AXIE grid by plotting the residual on the symmetry plane but these did not impact the signature extraction locations. The linear error transport equation method of Derlaga, Park, and Rallabhandi [60] was summarized. The error transport solution implicated the under resolved inlet shock that reflected off the wing and horizontal tail as a strong driver of the Euler $\mathrm{C} 25 \mathrm{~F}$ variation due to grid refinement. Their method incorporates atmospheric propagation via SBOOM and ground A-weighted sound exposure level calculation. The imprint of a wing tip conic structure onto the symmetry plane was also observed.

Participant D studied the impact of the $\kappa$ term in the Venkatakrishnan limiter [36] and chose a different $\kappa$ value for each configuration. Large $\kappa$ produced spurious oscillations that disappear with lower $\kappa$ values. They applied a multipole technique that dramatically reduced ground signatures differences between nearfield signatures at different extraction radii.

Participant E reported that low unstructured workshop grid surface resolution created high frequency disturbances near the body that persisted to the nearfield extraction locations. The unstructured grid produced an effective thickening of the boundary layer as compared the overset grid system. These overset grid systems used a slight misalignment to the freestream Mach angle to reduce spurious behavior of the high-order finite difference scheme.

Participant $\mathrm{F}$ also indicated that the workshop surface mesh produced pressure disturbances due to the unstructured triangle surface grid. The adaptive scheme to reduce estimated errors at sensor locations was required to resolve these surface grid facets, which lowered efficiency. These near body oscillations damped at larger distances from the model. These oscillations are suppressed with the introduction of a custom structured surface grid. Participant $F$ also gave details of the conic disturbance emanating form the wing tip vorticies. They showed extremely well-resolved symmetry plane solutions that highlight the complex plume shock interaction for the $\mathrm{C} 25 \mathrm{P}$ tail region.

Participant G produced the largest Euler simulations that showed small PL sensitivity to grid refinement for the finest grids. Details are also provided for the JWB workshop grid generation method. Participant H used equivalent area calculation method to study the three winged workshop models. They showed a large impact of multipole farfield correction.

Participant I compared nearfield and propagated ground signatures from the WS-Tet grids and multiple custom grids. The C25P case showed an expansion in the plume for the Pointwise custom grids that was not seen by other participants. The pressure field on the symmetry plane indicates this expansion initiates in the plume aft of the tail geometry. Participant $J$ examined the impact of flux function and reconstruction limiter in FUN3D for the C25F. The van Leer flux function with the van Albada reconstruction limiter was the most dissipative. Other combinations produced consistent results with only subtle differences. They used a space marching method [68] to reduce the grid requirements.

Participant K showed nonlinear error transport [69] of the JWB. They show very well-resolved nearfield pressure solutions based on controlling interpolation error or nearfield pressure target with goal-based anisotropic grid adaptation. They also provide resolution comparisons of the workshop-provided grids to the adapted grids with transverse grid slices. These slices show the complex shock structure above and below the $\mathrm{C} 25 \mathrm{~F}$.

\section{Discussion}

To reduce the burden and complexity of data submissions, the requested extraction locations were limited. Gathering AXIE off-centerline data could be used to verify that participant methods produced axisymmetric nearfield pressure fields. Multipole correction [30] was shown to reduce dependency on extraction distance by Participants D and G in their workshop presentations. Requesting data in a format that is compatible with multipole correction techniques would enable this correction to reduce the influence of extraction radius at the cost of increased submission and data 
reduction complexity. Larger offtrack angles would have less spanwise distortion and could be used to isolate spanwise distortion without a multipole correction.

Gathering the solution on the model surface, symmetry plane, transverse plane, or entire volume from participants may illustrate differences between submissions that contribute to differences at extraction locations. This additional information may connect nearfield signature differences to CFD scheme properties and grid construction. Renderings of these surfaces in participant talks was very illustrative. The availability of these 2D and 3D data sets would allow differences between submissions or ensemble statistics to be computed by interpolation to a common grid system.

A consistent nearfield signature windowing process was used in SBPW1 and SBPW2. The similarities in the nearfield SBPW2 signatures and the uniform model size allow a single nondimensional window for all models and extraction locations. Most methods produce freestream conditions in the forward portion of the signature, which reduces the influence of windowing there. The aft portion of the signature is typically more sensitive to windowing. This is due to a number of factors. The aft shock of a these models can be a significant contributor to overall loudness as seen in the SBPW1 optional complex configuration, where extremely minor nearfield signature differences resulted in uncoalesced or coalesced aft shock on the ground. This aft shock sensitivity is due to a historic emphasis and maturity of forward signature shaping. Techniques to shape aft signatures are a recent development. The aft portion of the signature returns to freestream over many body lengths. The use of extended CFD aft domains to fully capture this behavior is inefficient. Whitham [70] proposed a pressure distribution behind the rear shock to account for this asymptotic behavior, but it requires an estimate of the equivalent area. Linear ramping is used, consistent with SBPW1, because this windowing procedure is defined solely by geometry and is easier to replicate.

Discretizing curved surfaces with unstructured linear triangles can result in poor representation of the underlying continuous geometry surface normal. An equivalent problem is accurately resolving solution gradients, as examined by Shewchuk [71]. Inaccurate or noisy surface normals can result in small shocks and expansions at every surface slope change in supersonic flow. This can be mitigated with structured-grid generation in the curvature direction as shown in the workshop presentation of Participants E and F. Anisotropic grid generation based on curvature would reduce normal errors when compared to uniform isotropic triangles. Higher-order (quadratic) triangles could reduce surface normal errors but would require additional implementation in grid generation and flow solvers to accommodate higher-order surface fidelity.

The outer boundary of the workshop-provided grids was close enough to the five body length extraction location that farfield boundary reflections impacted the submissions of some participants. This issue was most noticeable in aft portions of signatures at five body lengths as identified in the presentation of Participant A. The boundary proximity placed requirements on the participant code farfield boundary condition implementation. Increasing the outer boundary extent would eliminate this concern for an increase in collar grid sizes.

\section{A. Flux Functions, Gradient Reconstruction, Reconstruction Limiters, and Grids}

NASA 2008 workshop summary [4] observed, "The two adjoint-based adaptation techniques produced identical signatures, except in the extrema, where limiter behavior is important or where the boundary conditions or geometry differed." Impact of limiters on nearfield pressure signatures was also studied by Park [72]. Those observations are based on signatures with coalesced $\mathrm{N}$-waves on the ground, making them louder than the SBPW2 model ground signatures. The issues related to accurate convective discretization are more important for the quieter signatures of SBPW2 that contain important expansions, shock-expansion cancellations, higher spatial frequency content, and other important smooth regions.

Participant A indicated that the Green-Gauss gradient reconstruction method was required for DLR-TAU on the workshop-provided grids. Participant D indicated that the topology of the collar grid was not ideal and that the solution was impacted by the limiter setting in JTAS. FUN3D has many flux function and reconstruction limiter options exercised by Participants C, G, and J. There were a variety of schemes exercised in submissions and other investigations documented in participant presentations. Differences were pronounced for the JWB and C25F due to the chioce of convective scheme. The inviscid FUN3D with van Albada reconstruction limiter case CC was low in PL on coarser grids than the unlimited reconstruction CD for the JWB. The van Albada limiter implementation in FUN3D has a dependency on the size of grid units [39], which is meters for the SBPW2 grids. Nikbay et al. [73] also observed a dependency on limiter, but this effect may be amplified on coarser grids. The variability due to gradient reconstruction and reconstruction limiting may indicate that additional research into the discretization of convective terms should be explored for finite-volume methods.

All these issues are closely related to the grids used, in particular cell quality and density. There are significant impacts of higher cell aspect ratios, nonsmooth size transitions and directional alignment with the Mach angle on the level of discretization error and spurious solution artifacts. While for some cases the spatial convergence of the 
signatures is excellent with a relatively small amount of discretization error on the finest meshes, see for example inviscid $\mathrm{C} 25 \mathrm{~F}$ at $\mathrm{R}=5$ and higher off-track angles, in many other cases there are significant differences in the fine mesh signatures with diverging error estimates.

\section{Progress Between SBPW1 and SBPW2}

A majority of the recommendations from the SBPW1 summary have been addressed in the execution of SBPW2. Iterative convergence and force and moment data was collected for SBPW2. The length units were standardized to meters and geometry and grids were provided in an orientation that did not require rotation before simulation. Uniformlyrefined grid systems were provided for all required and optional cases. Adaptive grid submissions were requested for final adapted and intermediate coarser grids to establish adaptive grid convergence trends. The required SBPW1 models produced coalesced $\mathrm{N}$-wave signatures on the ground. The use of quieter models with shaped signatures that persist to the ground has introduced new challenges for SBPW2. A range of geometric complexities that produce similar nearfield signatures was used in SBPW2. The concern about undershoots and overshoots at discontinuities has grown into a larger concern about convective discretization (gradient reconstruction and reconstruction limiting). This new concern is likely the result of the more resolved meshes available for SBPW2 and models that use complex shock and expansion cancellation processes to produce shaped signatures that persist to the ground.

The mean PL of fine grid submissions is much lower. The SBPW1 mean fine grid axisymmetric PL was 91.8 $\mathrm{dB}$, and the mean fine grid wing body was 95.5. The SBPW2 mean fine grid axisymmetric PL was $77.7 \mathrm{~dB}$, and the mean fine grid wing body was 79.4 for Euler submissions. This is a significant reduction because PL is a logarithmic function of sound energy. The logarithmic behavior of PL makes standard deviation difficult to compare between the two workshops. The SBPW1 fine grid axisymmetric PL standard deviation was $0.3 \mathrm{~dB}$, and the wing body standard deviation was 0.2. The SBPW2 fine grid axisymmetric PL standard deviation was $0.6 \mathrm{~dB}$, and the wing body was 1.4 for Euler submissions. The lower mean PL of SBPW2 make it more relevant to analysis that would support a certification standard for sonic boom. An acceptable PL limit is still being developed, but existing research indicates that a PL greater than $90 \mathrm{~dB}$ will likely be unacceptable.

The SBPW2 models are more complex than SBPW1, and more submissions were provided by participants. Workshop-provided grids and statistics are available for the required flow-through nacelle full configuration. The equivalent SBPW1 flow-through nacelle full configuration lacked sufficient number of submissions, and the variation was too large to produce meaningful statistics. There are a sufficient number of submissions to compute meaningful statistics for the required flow-through nacelle full configuration and optional full configuration with propulsion boundary conditions.

\section{Recommendations and Next Steps}

The NASA Supersonics Project sonic boom goal for future supersonic aircraft is 65 to 70 PL (dB) [74]. Henne [15] proposes a small quiet supersonic jet (QSJ) with 68 to 85 PL (dB). Using models with PL values near $80 \mathrm{~dB}$ in SBPW2 uncovered many issues not seen in SBPW1. Setting a target of 75 or lower may continue to pressure analysis and design methods to maintain the clear pace of international community improvement. A $75 \mathrm{~dB}$ PL target would also continue to address a relevant vehicle concept with an acceptability that could contribute to the formulation of a certification standard.

Having a range of geometric complexity was important to the participants of SBPW1 and SBPW2. A future workshop should continue to include both an axisymmetric configuration and a full-configuration (wing, body, tail, and nacelle) with complex nearfield signatures that produce mid-70s PL on the ground. Having an axisymmetric configuration that produces a signature with the complexity of the full configuration may help to isolate geometry complexity as a source of PL variation. The complex interaction and reflection between the C25F and C25P inlet, tail, nozzle, and plume is representative of the challenges of a full configuration. The shock/plume interaction experiment combines wind tunnel measurements [75] with existing comparisons to simulation [76]. This data set may provide a configuration to isolate these complex shock interaction effects. A formation of multiple axisymmetric bodies may isolate the spanwise distribution of acoustic disturbances as a source of PL variation.

Unfortunately, a cursory survey of publicly available geometries with wind tunnel measurements did not yield a sufficiently quiet configuration. This lack of available data opens up the possibility of performing the next workshop on a new configuration. The Low Boom Flight Demonstration Quiet Supersonic Transport (QueSST) X-plane may be a candidate with flight and possibly wind tunnel data in a time frame that is compatible with a future workshop.

The uniformly-refined workshop-provided grids were extremely valuable for SBPW1 and SBPW2. Future workshops should provide these grid systems for the required and optional test cases to encourage the widest participation. A balance must be made for data requests. The more data that is requested can aid comparisons between methods but 
increases the burden for participants. Planes or volumes of solution data should be requested to augment the current extraction line submissions. This would allow differences in nearfield signatures to be connected to regional differences in the solution. Symmetry plane and transverse plane solutions should be mandatory with optional volume solutions. The Helicopter Hover Prediction Workshop 2016 partnered with solution visualization experts to evaluate participant volume solutions [77]. Future workshops should also request data in a format that enables multipole correction to reduce the dependency on extraction location.

Establishing sonic boom prediction benchmark cases in the form of the Turbulence Modeling Resource Website $[78,79]$ is advocated by the participants. The SBPW1 and SBPW2 data sets are available and could form a critical mass of models and test cases. New SBPW2 participants used the SBPW1 case description and submission database to prepare for SBPW2 participation, which demonstrates its utility [35,38]. Establishing this initial sonic boom prediction benchmark resource would need to focus on making this existing data easy to access. A combined website, case description, and submitted results repository is being established by the Unstructured Grid Adaptation Working Group that could serve as a low overhead template for a sonic boom benchmark [80].

\section{Conclusions}

A summary and statistical analysis is provided for the Second AIAA Sonic Boom Workshop. There are three required models of increasing complexity: AXIE, JWB, and C25F. The C25P configuration with propulsion boundary conditions is optional. These models are designed with similar nearfield signatures to isolate geometry and shock/expansion interaction effects from propagation effects in the nearfield. Mean nearfield signatures at three body lengths are shown to reinforce this similarity and representative Euler symmetry plane solutions are provided to illustrate the range of complexity and flow interaction these models provide.

Eleven international participant groups submitted extracted nearfield signatures with forces, moment, and interative convergence norms. Government agencies, industry, and academia from five countries are represented in these groups. These participants optionally provided propagated ground and ground loudness levels. Pointwise statistics of the nearfield signatures are computed by interpolating to common $1 \mathrm{~cm}$ resolution. The nearfield submissions are propagated to the ground and noise levels are computed. This allows the grid convergence and statistical distribution of a noise levels to be computed with a uniform application of atmospheric propagation. Descriptive statistics of PL are provided in the form of box, whisker, and violin plots. The complete data set is available from the workshop FTP server to enable independent analysis, which is strongly encouraged. This data set is also used by participants in their publications. Grid convergence of these nearfield signatures is also analyzed with Richardson extrapolation.

The combination of nearfield statistics, nearfield grid convergence, PL convergence, and fine grid PL statistics provides a complete picture into the state of the art in nearfield sonic boom prediction by this international group of participants. The IQR contains the middle $50 \%$ of participants. Outliers are defined as 1.5 times the length of the IQR outside of the IQR. If the probability distribution is normal, outliers would be outside a 95\% likelihood. The AXIE model had the smallest IQR, but it increased dramatically at five body lengths. The JWB had a larger IQR than the AXIE, and JWB outliers were at a larger distance from the median. C25F had more outliers but a smaller IQR than the C25P. The IQR was $1 \mathrm{~dB}$ or less for the majority of extraction locations, and the median PL varied between 82 and $72 \mathrm{~dB}$, where the median PL dropped with offtrack angle for angles 30 degrees or greater. Median centerline PL was in the range of 80 to $78 \mathrm{~dB}$.

Progress since the first workshop was identified, where a majority of recommendations were accommodated including much quieter configurations. The complete configuration with a flow-through nacelle showed the most dramatic improvement between the two workshops. The current workshop cases are more relevant to vehicles with low annoyance than the first workshop. The models with quieter ground noise levels than the first workshop exposed weaknesses in analysis methods. Moving to even quieter configurations for a subsequent workshop would continue to pressure analysis methods to improve. New recommendations for potential improvements to the analysis methods and a possible subsequent workshop are provided. These recommendations include developing quieter test cases, including shock/plume interaction unit problems, and requesting more of the nearfield solution from participants. A proposal is made for maturing the the data sets from SBPW1 and SBPW2 into a benchmark website resource to encourage new entrants into sonic boom prediction and to foster improved analysis techniques.

\section{Appendix A: C25D and C25F Grid Generation Guidelines}

See Park et al. [66] for a survey of available techniques and the process used for SBPW1 and SBPW2. All lengths are in meters. The linear dimensions should be scaled linearly, growth rates should be scaled logrithmically, and the number of layers should be scaled inversely to produce a uniformly-refined or coarsened grid, i.e.,

scale $=1.0$ 


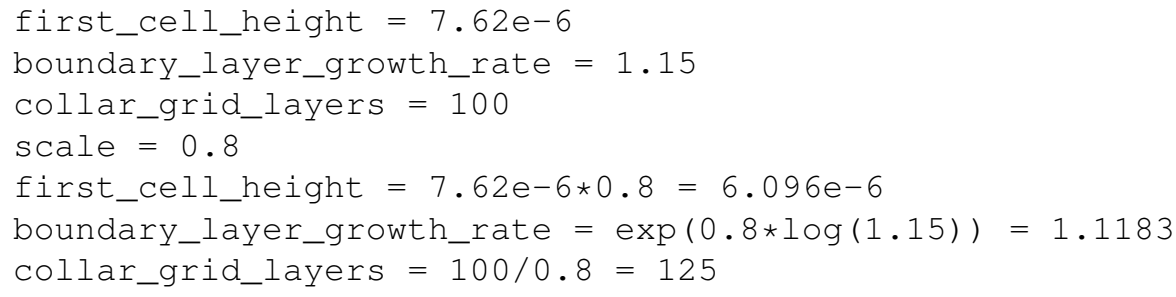

The core and collar grids should extend 1.5-2 body lengths aft of the Mach cone that extends from the tail of the configuration. The transition between an unstructured near-body grid and a Mach cone aligned collar grid should be made as close to the body as possible. The first cell in the collar grid should be of similar size to the adjacent core grid cell. Spacing in the radial direction should increase geometrically. The collar grid geometric growth rate should be scaled logrithmically (like the boundary layer growth rate) on coarsened or refined grids. The collar grid should be swept at the freestream mach angle. The collar grid should extend a minimum of 190 meters from the body to allow for the extraction of signatures at 5 body lengths. Prism or hexagon elements are preferred over tetrahedra in the collar grid to minimize signal attenuation in the collar grid.

The spacing at the nose of the configuration should be $15 \mathrm{~mm}$ and grow to $76 \mathrm{~mm}$ near the cockpit. It is best to reduce the deviation between the surface normals of the lower surface grid and the normals of the geometry, but this is difficult to control with unstructured grid.

The fuselage and center of the wing and tails should be resolved to $76 \mathrm{~mm}$. The minimum wing leading and trailing edge spacings should be $3 \mathrm{~mm}$. The inlet lip and exhaust nozzle edge and plug should be resolved to $6 \mathrm{~mm}$. The minimum horizontal and vertical tail leading and trailing edge spacings should be $6 \mathrm{~mm}$. The pod at the top of the horizontal tail should be resolved to $24 \mathrm{~mm}$. The core grid spacing should increase slowly to towards the core grid and collar grid transition (constant spacing is ideal if affordable) to propagate disturbances from the aircraft to the collar grid. The exhaust plume of the $\mathrm{C} 25 \mathrm{P}$ should be resolved to $38 \mathrm{~mm}$ and spacing should increase very slowly down stream (constant spacing is ideal if affordable) to prevent an artificial compression due to abrupt plume dissipation.

\section{Acknowledgments}

The authors would like to thank the entire SBPW2 organizing committee, participants, and attendees. The discussions during the workshop and in the hallways helped to craft the content and recommendations of this summary. The C25F and C25P configurations were developed by Mathias Wintzer, Irian Ordaz, James W. Fenbert, and Sriram Rallabhandi The JWB was developed by Atsushi Ueno, Masashi Kanamori, and Yoshikazu Makino. George Anderson and Mike Aftosmis performed the inverse design for the AXIE geometry. The geometry preparation, translation, and evaluation was performed by Scott Brynildsen, Bill Jones, and Sriram Rallabhandi. Andrew Clemens created the JWB grid systems. Marie Denison and Mike Aftosmis provided feedback on early iterations of the workshop volume and surface grids. Jeffrey Housman reported an error in JWB geometry orientation that was corrected before it impacted other participants.

The suggestions provided by SBPW1 participants [1] contributed greatly to the success of SBPW2. Sriram Rallabhandi provided the atmospheric propagation tool sBOOM and assistance on its use. Alexandra Loubeau provided the noise level calculation tool, guidance on its use, and detailed background on noise levels. Marie Denison, Kyle Thompson, Bil Kleb, and Steve Bauer provided valuable feedback on this manuscript. This work is supported under the Commercial Supersonic Transport Project of NASA's Fundamental Aerodynamics Program.

\section{References}

[1] Park, M. A. and Morgenstern, J. M., "Summary and Statistical Analysis of the First AIAA Sonic Boom Prediction Workshop," AIAA Journal of Aircraft, Vol. 53, No. 2, March-April 2016, pp. 578-598. doi:10.2514/1.C033449.

[2] Rallabhandi, S. K. and Loubeau, A., "Propagation Summary and Statistical Analysis of the Second AIAA Sonic Boom Prediction Workshop," AVIATION 2017, American Institute of Aeronautics and Astronautics, Reston, VA (submitted for publication).

[3] Hemsch, M. J., "Statistical Analysis of Computational Fluid Dynamics Solutions from the Drag Prediction Workshop," AIAA Journal of Aircraft, Vol. 41, No. 1, 2004, pp. 95-103.

[4] Park, M. A., Aftosmis, M. J., Campbell, R. L., Carter, M. B., Cliff, S. E., and Bangert, L. S., "Summary of the 2008 NASA Fundamental Aeronautics Program Sonic Boom Prediction Workshop," AIAA Journal of Aircraft, Vol. 51, No. 3, May-June 2014, pp. 987-1001. doi:10.2514/1.C032589.

[5] Park, M. A. and Morgenstern, J. M., "Summary and Statistical Analysis of the First AIAA Sonic Boom Prediction Workshop," AIAA Paper 2014-2006, 2014. 
[6] Levy, D. W., Laflin, K. R., Tinoco, E. N., Vassberg, J. C., Mani, M., Rider, B., Rumsey, C. L., Wahls, R. A., Morrison, J. H., Brodersen, O. P., Crippa, S., Mavriplis, D. J., and Murayama, M., "Summary of Data from the Fifth Computational Fluid Dynamics Drag Prediction Workshop,” AIAA Journal of Aircraft, Vol. 51, No. 4, July-Aug. 2014, pp. 1194-1213. doi:10.2514/1.C032389.

[7] Morrison, J. H., "Statistical Analysis of the Fifth Drag Prediction Workshop Computational Fluid Dynamics Solutions," AIAA Journal of Aircraft, Vol. 51, No. 4, July-Aug. 2014, pp. 1214-1222. doi:10.2514/1.C032736.

[8] Rumsey, C. L. and Slotnick, J. P., "Overview and Summary of the Second AIAA High Lift Prediction Workshop," AIAA Journal of Aircraft, Vol. 52, No. 4, July-Aug. 2015, pp. 1006-1025. doi:10.2514/1.C032864.

[9] DeBonis, J. R., Oberkampf, W. L., Wolf, R. T., Orkwis, P. D., Turner, M. G., Babinsky, H., and Benek, J. A., "Assessment of Computational Fluid Dynamics and Experimental Data for Shock Boundary-Layer Interactions," AIAA Journal, Vol. 50, No. 4, April 2012, pp. 891-903. doi:10.2514/1.J051341.

[10] Kirz, J. and Rudnik, R., "DLR TAU Simulations for the Second AIAA Sonic Boom Prediction Workshop,” AVIATION 2017, American Institute of Aeronautics and Astronautics, Reston, VA (submitted for publication).

[11] Elmiligui, A., Carter, M. B., Nayani, S., Cliff, S., and Pearl, J., "USM3D Simulations for 2nd Sonic Boom Workshop," AVIATION 2017, American Institute of Aeronautics and Astronautics, Reston, VA (submitted for publication).

[12] Anderson, G. R., Aftosmis, M. J., and Nemec, M., "Cart3D Simulations for the Second AIAA Sonic Boom Prediction Workshop," AVIATION 2017, American Institute of Aeronautics and Astronautics, Reston, VA (submitted for publication).

[13] “Civil Aircraft Sonic Boom,” Code of Federal Regulations, Title 14, Pt. 91.817, 2011.

[14] Liebhardt, B., Linke, F., and Dahlmann, K., "Supersonic Deviations: Assessment of Sonic-Boom-Restricted Flight Routing," AIAA Journal of Aircraft, Vol. 51, No. 6, Nov.-Dec. 2014, pp. 1987-1996. doi:10.2514/1.C032591.

[15] Henne, P. A., "Case for Small Supersonic Civil Aircraft," AIAA Journal of Aircraft, Vol. 42, No. 3, May-June 2005, pp. 765774. doi:10.2514/1.5119.

[16] Sakata, K., "Japan’s Supersonic Technology and Business Jet Perspectives," AIAA Paper 2013-21, 2013.

[17] Morgenstern, J. M., "How to Accurately Measure Low Sonic Boom or Model Surface Pressures in Supersonic Wind Tunnels," AIAA Paper 2012-3215, 2012.

[18] Morgenstern, J. M., "Distortion Correction for Low Sonic Boom Measurement in Wind Tunnels," AIAA Paper 2012-3216, 2012.

[19] Roache, P. J., "Verification of Codes and Calculations," AIAA Journal, Vol. 36, No. 5, May 1998, pp. 696-702. doi:10.2514/2.457.

[20] Leatherwood, J. D., Sullivan, B. M., Shepherd, K. P., McCurdy, D. A., and Brown, S. A., "Summary of Recent NASA Studies of Human Response to Sonic Booms," The Journal of the Acoustical Society of America, Vol. 111, No. 1, Jan. 2002, pp. 586-598. doi:10.1121/1.1371767.

[21] Stevens, S. S., "Perceived Level of Noise by Mark VII and Decibels (E)," Journal of the Acoustical Society of America, Vol. 51, No. 2B, 1972, pp. 575-601. doi:10.1121/1.1912880.

[22] Loubeau, A., Naka, Y., Cook, B. G., Sparrow, V. W., and Morgenstern, J. M., "A New Evaluation of Noise Metrics for Sonic Booms Using Existing Data,” AIP Conference Proceedings, Vol. 1685, No. 1, 2015, pp. 090015. doi:10.1063/1.4934481.

[23] Shepherd, K. P. and Sullivan, B. M., "A Loudness Calculation Procedure Applied to Shaped Sonic Booms,” NASA TP-3134, Langley Research Center, Nov. 1991. doi:2060/19920002547.

[24] Rallabhandi, S. K., "Advanced Sonic Boom Prediction Using the Augmented Burgers Equation," AIAA Journal of Aircraft, Vol. 48, No. 4, July-Aug. 2011, pp. 1245-1253. doi:10.2514/1.C031248.

[25] Rallabhandi, S. K., "Sonic Boom Adjoint Methodology and its Applications," AIAA Paper 2011-3497, 2011.

[26] Rallabhandi, S. K., Nielsen, E. J., and Diskin, B., "Sonic Boom Mitigation Through Aircraft Design and Adjoint Methodology," AIAA Journal of Aircraft, Vol. 51, No. 2, March-April 2014. doi:10.2514/1.C032189.

[27] Cleveland, R. O., Propagation of Sonic Booms Through a Real, Stratified Atmosphere, Ph.D. thesis, University of Texas at Austin, May 1995. doi:2144/1443.

[28] U.S. Standard Atmosphere, 1976, U.S. Government Printing Office, 1976.

[29] American National Standards Institute, "Method for Calculation of the Absorption of Sound by the Atmosphere," ANSI S1.26-1995, Sept. 1995.

[30] Page, J. A. and Plotkin, K. J., "An Efficient Method for Incorporating Computational Fluid Dynamics into Sonic Boom Prediction,” AIAA Paper 91-3275, 1991.

[31] Derlaga, J. M. and Morrison, J. H., "Statistical Analysis of CFD Solutions from the 6th AIAA CFD Drag Prediction Workshop,” AIAA Paper 2017-1209, 2017. 
[32] McGill, R., Tukey, J. W., and Larsen, W. A., "Variations of Box Plots," The American Statistician, Vol. 1, No. 32, 1978, pp. 12-16. doi:10.2307/2683468.

[33] Hintze, J. L. and Nelson, R. D., "Violin Plots: A Box Plot-Density Trace Synergism," The American Statistician, Vol. 2, No. 52, 1998, pp. 181-184. doi:10.1080/00031305.1998.10480559.

[34] Schwamborn, D., Gerhold, T., and Heinrich, R., "The DLR TAU-Code: Recent Applications in Research and Industry," European Conference on Computational Fluid Dynamics ECCOMAS CFD 2006, Sept. 2006.

[35] Kirz, J. and Rudnik, R., "DLR Simulations of the First AIAA Sonic Boom Prediction Workshop Cases," AIAA Paper 20170276, 2017.

[36] Venkatakrishnan, V., "Convergence to Steady State Solutions of the Euler Equations on Unstructured Grids with Limiters," Journal of Computational Physics, Vol. 118, No. 1, 1995, pp. 120-130.

[37] Barth, T. J. and Jespersen, D. C., "The Design and Application of Upwind Schemes on Unstructured Meshes," AIAA Paper 89-366, 1989.

[38] Ma, B., Wang, G., Ren, J., Zhengyin, Y., and Zha, G., "Near Field Sonic Boom Analysis with HUNS3D Solver," AIAA Paper 2017-0038, 2017.

[39] Biedron, R. T., Carlson, J.-R., Derlaga, J. M., Gnoffo, P. A., Hammond, D. P., Jones, W. T., Kleb, B., Lee-Rausch, E. M., Nielsen, E. J., Park, M. A., Rumsey, C. L., Thomas, J. L., and Wood, W. A., "FUN3D Manual: 13.1,” NASA TM-2016219580, Langley Research Center, Feb. 2017. doi:2060/20170002585.

[40] Roe, P. L., "Approximate Riemann Solvers, Parameter Vectors, and Difference Schemes," Journal of Computational Physics, Vol. 43, No. 2, Oct. 1981, pp. 357-372. doi:10.1016/0021-9991(81)90128-5.

[41] van Albada, G. D., van Leer, B., and Roberts, Jr., W. W., "A Comparative Study of Computational Methods in Cosmic Gas Dynamics," Astronomy and Astrophysics, Vol. 108, 1982, pp. 76-84.

[42] van Leer, B., "Towards the Ultimate Conservative Difference Scheme. II. Monotonicity and Conservation Combined in a Second-Order Scheme," Journal of Computational Physics, Vol. 14, No. 4, March 1974, pp. 361-370. doi:10.1016/00219991(74)90019-9.

[43] Toro, E. F., Spruce, M., and Speares, W., "Restoration of the Contact Surface in the HLL-Riemann Solver," Shock Waves, Vol. 4, No. 1, 1994, pp. 25-34. doi:10.1007/BF014146292.

[44] Nakahashi, K., Ito, Y., and Togashi, F., “Some challenges of realistic flow simulations by unstructured grid CFD," International Journal for Numerical Methods in Fluids, Vol. 43, No. 6-7, Nov. 2003, pp. 769-783. doi:10.1002/fld.559.

[45] Kiris, C. C., Housman, J. A., Barad, M. F., Sozer, E., Brehm, C., and Moini-Yekta, S., "Computational framework for Launch, Ascent, and Vehicle Aerodynamics (LAVA)," Aerospace Science and Technology, Vol. 55, Aug. 2016, pp. 189-219. doi:10.1016/j.ast.2016.05.008.

[46] Housman, J. A., Sozer, E., Moini-Yekta, S., and Kiris, C. C., "LAVA Simulations for the First AIAA Sonic Boom Prediction Workshop," AIAA Paper 2014-2008, 2014.

[47] Aftosmis, M. J. and Nemec, M., “Cart3D Simulations for the First AIAA Sonic Boom Prediction Workshop," AIAA Paper 2014-558, 2014.

[48] Howe, D. C., "Hybrid CART3D/OVERFLOW Near-Field Analysis of a Low Boom Configuration with Wind Tunnel Comparisons," AIAA Paper 2011-3336, 2011.

[49] Chakravarthy, S., "A unified-grid finite volume formulation for computational fluid dynamics," International Journal for Numerical Methods in Fluids, Vol. 32, No. 1, Sept. 1999, pp. 309-323. doi:10.1002/(SICI)10970363(19990915)31:1<309::AID-FLD971>3.0.CO;2-M.

[50] Frink, N. T., Pirzadeh, S. Z., Parikh, P., Pandya, M. J., and Bhat, M. K., "The NASA Tetrahedral Unstructured Software System," The Aeronautical Journal, Vol. 104, No. 1040, Oct. 2000, pp. 491-499.

[51] Menier, V., Loseille, A., and Alauzet, F., “CFD Validation and Adaptivity for Viscous Flow Simulations,” AIAA Paper 20142925, 2014.

[52] Loseille, A., Frazza, L., and Alauzet, F., "Comparing anisotropic adaptive strategies on the 2nd AIAA sonic boom workshop geometry,” AIAA Paper 2017-0281, 2017.

[53] Salah El Din, I., Dagrau, F., and Loseille, A., "Computational and Experimental Assessment of Models for the First AIAA Sonic Boom Prediction Workshop Using High Fidelity CFD Methods with Mesh Adaptation,” AIAA Paper 2014-2009, 2014.

[54] Debiez, C. and Dervieux, A., "Mixed-Element-Volume MUSCL Methods with Weak Viscosity for Steady and Unsteady Flow Calculations," Computers and Fluids, Vol. 29, No. 1, Jan. 2000, pp. 89-118. doi:10.1016/S0045-7930(98)00059-0.

[55] Spalart, P. R. and Allmaras, S. R., “A One-Equation Turbulence Model for Aerodynamic Flows," La Recherche Aérospatiale, Vol. 1, 1994, pp. 5-21.

[56] Allmaras, S. R., Johnson, F. T., and Spalart, P. R., "Modifications and Clarifications for the Implementation of the SpalartAllmaras Turbulence Model," Seventh International Conference on Computational Fluid Dynamics (ICCFD7), 2012.

60 of 61 
[57] Shih, T.-H., Liou, W. W., Shabbir, A., Yang, Z., and Zhu, J., "A New k- $\epsilon$ Eddy Viscosity Model for High Reynolds Number Turbulent Flows," Computers and Fluids, Vol. 24, No. 3, March 1995, pp. 227-238. doi:10.1016/0045-7930(94)00032-T.

[58] Wintzer, M., Ordaz, I., and Fenbert, J. W., "Under-Track CFD-Based Shape Optimization for a Low-Boom Demonstrator Concept," AIAA Paper 2015-2260, 2015.

[59] Ordaz, I., Wintzer, M., and Rallabhandi, S. K., "Full-Carpet Design of a Low-Boom Demonstrator Concept,” AIAA Paper 2015-2261, 2015.

[60] Derlaga, J. M., Park, M. A., and Rallabhandi, S. K., "Application of Exact Error Transport Equations and Adjoint Error Estimation to AIAA Workshops," AIAA Paper 2017-0076, 2017.

[61] Dannenhoffer, III, J. F., “OpenCSM: An Open-Source Constructive Solid Modeler for MDAO,” AIAA Paper $2013-701,2013$.

[62] Wintzer, M., Optimization and Adjoint-Based CFD for the Conceptual Design of Low Sonic Boom Aircraft, Ph.D. thesis, Stanford University, Aug. 2012.

[63] Ueno, A., Kanamori, M., and Makino, Y., "Multi-fidelity Low-boom Design Based on Near-field Pressure Signature,” AIAA Paper 2016-2033, 2016.

[64] Ordaz, I., Geiselhart, K. A., and Fenbert, J. W., "Conceptual Design of Low-Boom Aircraft with Flight Trim Requirement," AIAA Journal of Aircraft, Vol. 52, No. 3, May-June 2015, pp. 932-939. doi:10.2514/1.C033160.

[65] Anderson, G. R., Shape Optimization in Adaptive Search Spaces, Ph.D. thesis, Stanford University, Dec. 2015.

[66] Park, M. A., Campbell, R. L., Elmiligui, A., Cliff, S. E., and Nayani, S. N., "Specialized CFD Grid Generation Methods for Near-Field Sonic Boom Prediction,” AIAA Paper 2014-115, 2014.

[67] Dompierre, J., Labbé, P., Vallet, M.-G., and Camarero, R., "How to Subdivide Pyramids, Prisms, and Hexahedra into Tetrahedra," 8th International Meshing Roundtable, 1999.

[68] Shen, H. and Lazzara, D., "Prediction of Sonic Boom Signature Using Euler-Full Potential CFD with Grid Adaptation and Shock Fitting," AIAA Paper 2016-2037, 2016.

[69] Loseille, A., Dervieux, A., and Alauzet, F., "Anisotropic Norm-Oriented Mesh Adaptation for Compressible Inviscid Flows," AIAA Paper 2015-2037, 2015.

[70] Whitham, G. B., "The Flow Pattern of a Supersonic Projectile," Communications on Pure and Applied Mathematics, Vol. 5, No. 3, Aug. 1952, pp. 301-348. doi:10.1002/cpa.3160050305.

[71] Shewchuk, J. R., "What Is a Good Linear Element? Interpolation, Conditioning, and Quality Measures," 11th International Meshing Roundtable, Sandia National Laboratories, Sept. 2002, pp. 115-126.

[72] Park, M. A., Anisotropic Output-Based Adaptation with Tetrahedral Cut Cells for Compressible Flows, Ph.D. thesis, Massachusetts Institute of Technology, Sept. 2008. doi:1721.1/46363.

[73] Nikbay, M., Stanford, B., West, T., and Rallabhandi, S., "Impact of Aeroelastic Uncertainties on the Sonic Boom Signature of a Commercial Supersonic Transport Configuration," AIAA Paper 2017-0040, 2017.

[74] "Research Opportunities in Aeronautics - 2008 (ROA-2008)," Amendment 7 to the NASA Research Annoucnements (NRA), Appendix A-4 NNH08ZEA001N, March 2008.

[75] Durston, D. A., Cliff, S. E., Denison, M., Smith, N. T., Heineck, J. T., Schairer, E. T., Kushner, L. K., Castner, R. S., Elmiligui, A. A., Carter, M. B., Winski, C., Shea, P. R., and Blumenthal, B., "Nozzle Plume/Shock Interaction Sonic Boom Test Results from the NASA Ames 9- by 7-Foot Supersonic Wind Tunnel," AIAA Paper 2017-41, 2017.

[76] Jensen, J. C., Denison, M., Durston, D. A., and Cliff, S. E., "Computational Evaluations of Experimental Data for Sonic Boom Models with Nozzle Jet Flow Interactions," AIAA Paper 2017-42, 2017.

[77] Duque, E. P., Toyoda, A., Burklund, M. D., Hariharan, N., Narducci, R., and Stone, C. P., "Direct Comparison of Hover Prediction Workshop Results," AIAA Paper 2016-35, 2016.

[78] Rumsey, C. L., Smith, B. R., and Huang, G. P., "Description of a Website Resource for Turbulence Modeling Verification and Validation," AIAA Paper 2010-4742, 2010.

[79] Rumsey, C. L., "Recent Developments on the Turbulence Modeling Resource Website," AIAA Paper 2015-2927, 2015.

[80] Ibanez, D., Barral, N., Krakos, J., Loseille, A., Michal, T., and Park, M., "First Benchmark of the Unstructured Grid Adaptation Working Group," International Meshing Roundtable, Sandia National Laboratories, Albuquerque, NM (submitted for publication). 